\title{
Moments of Eisenstein series on convex co-compact hyperbolic manifolds
}

\author{
Patrick Munroe \\ Doctor of Philosophy \\ Department of Mathematics and Statistics
}

McGill University

Montreal, Quebec

August 12, 2015

A thesis submitted to McGill University in partial fulfillment of the requirements of the degree of Doctor of Philosophy

(C) Patrick Munroe, 2015 


\section{ACKNOWLEDGEMENTS}

I would like to thank my supervisor, Dmitry Jakobson, for the opportunity of working under his guidance and for his encouragement and support throughout all the stages of this work. I am also grateful to him for suggesting the problem addressed in this thesis.

It is a pleasure for me to thank Colin Guillarmou, to whom I am especially grateful for many helpful discussions, which contributed significantly to my general understanding of the field of research to which this work belongs. I must also thank him for his exceptional hospitality during my stay at École Normale Supérieure de Paris.

I would also like to thank Frédéric Naud for discussions and comments that were particularly helpful in the latter phase of the research.

Finally, I would like to thank my family and my friends for their constant encouragement and their presence in my life during the time of my graduate studies. 


\begin{abstract}
We investigate the high-energy limits of the moments of Eisenstein series, when these functions are considered as real random variables over a compact subset of a convex cocompact hyperbolic manifold. In the first part, under a restriction on the Hausdorff dimension of the limit set of the fundamental group of the manifold, we prove a general formula describing all the moments of the Eisenstein series at high-energy. In particular, we show that all the odd-order moments vanish. In the second part, we study the rate of convergence of the moments in the high-energy limit. In the case of the odd-order moments, we prove that the rate of convergence is at least polynomial. As for the even-order moments, following an approach based on the work of Guillarmou and Naud [16] concerning the equidistribution of Eisenstein series, we find a polynomial error term for the fourth moment under the additional hypothesis that the manifold in question is a surface.
\end{abstract}




\section{RÉSUMÉ}

Nous étudions les moments des séries d'Eisenstein dans la limite des hautes énergies, lorsque ces fonctions sont considérées en tant que variables aléatoires sur un sous-ensemble compact d'une variété hyperbolique convexe co-compacte. Dans un premier temps, en imposant une condition sur la dimension de Hausdorff de l'ensemble limite du groupe fondamental de la variété, nous obtenons une formule générale décrivant tous les moments des séries d'Eisenstein à hautes énergies. Dans un second temps, nous étudions la vitesse de convergence des moments dans la limite des hautes énergies. Dans le cas des moments d'ordre impair, nous démontrons que la vitesse de convergence est au moins polynomiale. En ce qui concerne les moments d'ordre pair, en suivant une approche basée sur les travaux de Guillarmou et Naud [16] concernant l'équidistribution des séries d'Eisenstein, nous trouvons un terme d'erreur d'ordre polynomial pour le quatrième moment en ajoutant l'hypothèse que la variété en question est une surface. 


\section{TABLE OF CONTENTS}

ACKNOWLEDGEMENTS ......................... ii

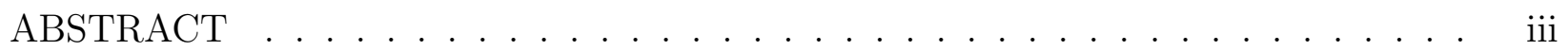

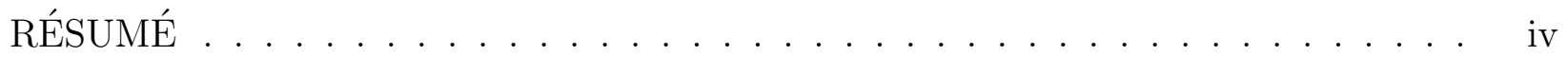

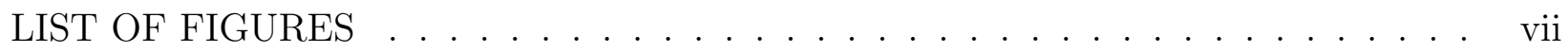

1 Introduction . . . . . . . . . . . . . . . . . 1

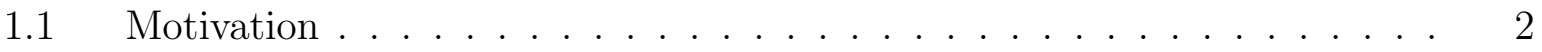

1.2 Presentation of the results . . . . . . . . . . . . . . . . . . . 4

1.3 Organization of the thesis . . . . . . . . . . . . 8

2 Preliminaries .......................... . . . 10

2.1 Hyperbolic manifolds . . . . . . . . . . . . . . . . . . . . . . . . 10

2.1.1 Möbius transformations . . . . . . . . . . . . . . . . . 11

2.1 .2 Limit set . . . . . . . . . . . . . . . . . . . . . . . . . . . . . . . . . . 17

2.1.3 Geometrically finite manifolds . . . . . . . . . . . . . 18

2.1.4 Convex co-compact manifolds . . . . . . . . . . . . . . . 20

2.2 Spectral theory of infinite-volume hyperbolic manifolds . . . . . . . . . 22

2.2.1 Finite volume . . . . . . . . . . . . . . . . . . 22

2.2 .2 Spectral theorem . . . . . . . . . . . . . . . . 26

2.2 .3 Poincaré series . . . . . . . . . . . . . . . . . . . . . . . . . . . . . . . . . . . . 26

2.2.4 Eisenstein series . . . . . . . . . . . . . . . . . . . 28

3 The moments at high-energy . . . . . . . . . . . . . . . . . . . 34

3.1 Proof of Theorem $1.1 \ldots \ldots \ldots \ldots$. . . . . . . . . . . . . . . . . . . . . . . . . . 34

3.1 .1 The sum over $S_{2} \ldots \ldots \ldots$. . . . . . . . . . . 36

3.1 .2 The sum over $S_{1} \ldots \ldots \ldots$. . . . . . . . . . . . 38

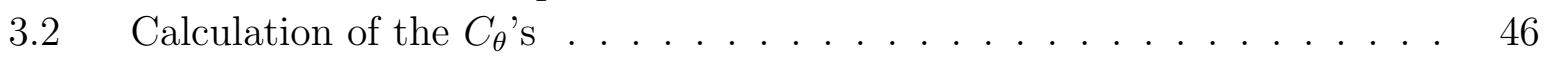

3.2.1 Example: $p=6$. . . . . . . . . . . . . . . . 46

3.2.2 Example: $p=8$. . . . . . . . . . . . . . . . 47

3.2 .3 The hyperbolic space $X=\mathbb{H}^{n+1} \ldots \ldots \ldots$. . . . . . . . 50

3.3 The moments of $F_{\lambda} \ldots \ldots \ldots \ldots$

4 An error term for the odd-order moments . . . . . . . . . . . . . . 52

$4.1 \quad$ Proof of Theorem $1.2 \ldots \ldots \ldots \ldots$. . . . . . . . . . . . . . . . . . . 
4.1.1 Finding the zero set of $\nabla_{m} \psi_{\gamma} \ldots \ldots \ldots \ldots \ldots \ldots$

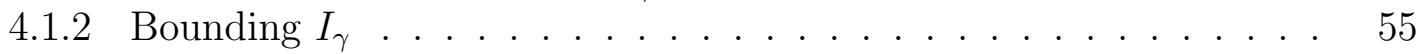

4.1.3 Summing over the elements of $\Gamma \ldots \ldots \ldots \ldots$

$4.2 \quad$ An improved error term for the third moment on surfaces . . . . . . . . 63

4.2 .1 Proof of Proposition 4.1 . . . . . . . . . . . . . . . . 65

5 An error term for the fourth moment on surfaces . . . . . . . . . . . . 71

5.1 The second moment . . . . . . . . . . . . . . . . . . . . . 72

5.2 Proof of Theorem $1.3 \ldots \ldots \ldots \ldots$

5.2.1 Case 1: $\gamma_{1}^{\prime}=\gamma_{2}$ or $\gamma_{2}^{\prime}=\gamma_{1} \ldots \ldots \ldots \ldots \ldots$

5.2.2 Case 2: The elements $\gamma_{1}, \gamma_{2}, \gamma_{1}^{\prime}$ and $\gamma_{2}^{\prime}$ are all different. . . . . 78

5.2.3 Case 3: Either $\gamma_{1}^{\prime}=\gamma_{2}^{\prime}$ and $\gamma_{1} \neq \gamma_{2}$ or $\gamma_{1}^{\prime} \neq \gamma_{2}^{\prime}$ and $\gamma_{1}=\gamma_{2} . \ldots .85$

5.3 Proof of Corollary $1.5 \ldots \ldots \ldots \ldots$

6 Conclusion . . . . . . . . . . . . . . . . . . . . . . . . . 92

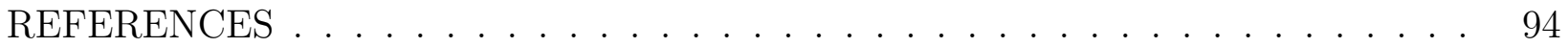




\section{LIST OF FIGURES}

3-1 The lattice of partitions of a set of 3 elements. . . . . . . . . . . . . . 46

3-2 The lattice of partitions of a set of 4 elements. . . . . . . . . . . . . . 48 


\section{CHAPTER 1 Introduction}

For $n \geq 1$, let $\mathbb{H}^{n+1}$ be the $(n+1)$-dimensional hyperbolic space and let $\operatorname{Isom}_{+}\left(\mathbb{H}^{n+1}\right)$ denote the group of orientation-preserving isometries of $\mathbb{H}^{n+1}$. Let $\Gamma$ be a torsion-free discrete subgroup of $\operatorname{Isom}_{+}\left(\mathbb{H}^{n+1}\right)$. The set of limit points of all the orbits of the action of $\Gamma$ on $\mathbb{H}^{n+1}$, i.e., $\Gamma z$ for $z \in \mathbb{H}^{n+1}$, consists of a subset $\Lambda_{\Gamma}$ of $\partial \mathbb{H}^{n+1}$, which will be referred to as the limit set of $\Gamma$.

In this thesis, we are interested in quotients of the form $X:=\Gamma \backslash \mathbb{H}^{n+1}$ giving rise to infinite volume hyperbolic manifolds without cusps. More precisely, the group $\Gamma$ has to satisfy the additional hypothesis that it admits a finite-sided convex polygonal fundamental domain whose closure does not intersect the limit set of $\Gamma$. The resulting quotient $X$ is then said to be convex co-compact.

Endowed with the hyperbolic metric inherited from $\mathbb{H}^{n+1}$, the Riemannian manifold $X$ is not compact. However, it is conformally compact, in the sense that it can be compactified to a smooth manifold $\bar{X}$ with boundary $\partial \bar{X}$ through the multiplication of its metric by some conformal factor. For precise definitions and for further explanation of the concepts introduced above, see $\S 2.1$.

Since the work of Lax and Phillips [22, 23], it is a well-known fact that the spectrum of the Laplacian on geometrically finite hyperbolic manifolds of infinite volume decomposes into an absolutely continuous part $\left[n^{2} / 4, \infty\right)$ and a (possibly empty) finite set of eigenvalues in the interval $\left(0, n^{2} / 4\right)$. The absolutely continuous part of the spectrum is represented by the Eisenstein series $E\left(s_{\lambda} ; m, \xi\right)$ with $s_{\lambda}:=\frac{n}{2}+i \lambda, m \in X$ and $\xi \in \partial \bar{X}$. These functions are generalized eigenfunctions of the Laplacian in the sense that they are non- $L^{2}$ functions 
which satisfy the usual eigenvalue equation:

$$
\Delta_{X} E(s ; m, \xi)=s(n-s) E(s ; m, \xi)
$$

The reader who is not familiar with the spectral theory of infinite-volume hyperbolic manifolds is encouraged to read $§ 2.2$ in which a brief exposition of some important results in this field can be found. See also $§ 2.2 .4$ for a precise definition of the Eisenstein series.

\subsection{Motivation}

Using the fact that $E(s ; m, \xi)$ enjoys a representation as an absolutely convergent series when the real part of the spectral parameter $s \in \mathbb{C}$ is large enough (see Lemma 2.20 below), Guillarmou and Naud [16] showed the equidistribution of Eisenstein series on convex cocompact manifolds $X$ when the Hausdorff dimension of the limit set, $\delta_{\Gamma}:=\operatorname{dim}_{\mathrm{Haus}}\left(\Lambda_{\Gamma}\right)$, is small enough. More precisely, they proved that if $\delta_{\Gamma}<n / 2$, then for any smooth compactly supported function $a$ on $X$, i.e., $a \in C_{0}^{\infty}(X)$, we have

$$
\int_{X} a(m)\left|E\left(s_{\lambda} ; m, \xi\right)\right|^{2} d v_{X}(m)=\int_{X} a(m) E(n ; m, \xi) d v_{X}(m)+\mathcal{O}\left(\lambda^{2 \delta_{\Gamma}-n}\right),
$$

as $\lambda \rightarrow \infty$, where $d v_{X}(m)$ is the hyperbolic volume measure.

Inspired by this result, we have set as an objective to study the "high-energy" limit of all the moments of the real and imaginary parts of Eisenstein series, when seen as real random variables over a compact subset of a convex co-compact manifold. To be more precise, fix a

nice compact set $K \subset X$ with non-empty interior and let $a \in C_{0}^{\infty}(X)$ be such that $a(m) \geq 0$ for all $m \in \operatorname{supp}(a) \subset K$ and

$$
\int_{X} a(m) d v_{X}(m)=1
$$

If we define $F_{\lambda}(m, \xi)$ to be either $\operatorname{Re}\left(E\left(s_{\lambda} ; m, \xi\right)\right)$ or $\operatorname{Im}\left(E\left(s_{\lambda} ; m, \xi\right)\right)$, then $\left(F_{\lambda}\right)_{\lambda \in \mathbb{R}_{+}}$can be seen as a family of real-valued random variables with respect to the probability measure 
$a(m) d v_{X}(m)$ on $X$. By definition, the $p$-th moment of the random variable $F_{\lambda}$ is given by

$$
\mathbb{E}\left(F_{\lambda}^{p}(\cdot, \xi)\right)=\int_{X}\left(F_{\lambda}(m, \xi)\right)^{p} a(m) d v_{X}(m)
$$

Our goal is to gain a better understanding of the high-energy limit, i.e., the limit $\lambda \rightarrow \infty$, of $\mathbb{E}\left(F_{\lambda}^{p}(\cdot, \xi)\right)$. With a complete description of the high-energy limit of all the moments, one could expect to find a probability law whose $p$-th moment corresponds to $\lim _{\lambda \rightarrow \infty} \mathbb{E}\left(F_{\lambda}^{p}(\cdot, \xi)\right)$, which could eventually lead to a solution of the Hamburger moment problem. In other words, one could find a random variable $F$ (with respect to $a(m) d v_{X}(m)$ on $X$ ) such that $F_{\lambda}$ converges in distribution to $F$ as $\lambda \rightarrow \infty$. Such information would provide deep insights into the high-energy behaviour of those generalized eigenfunctions of the Laplacian.

The question of studying real eigenfunctions of the Laplacian in the limit $\lambda \rightarrow \infty$ has already been raised in the past. The famous Random Wave Conjecture of Berry [6] predicts that the real and imaginary parts of high-energy eigenfunctions should resemble random waves in classically chaotic systems. In the case of hyperbolic surfaces, many numerical tests were conducted to confirm the validity of such a model; see, e.g., [18]. However, only little progress was made towards this conjecture. Among the noteworthy results for the odd-order moments is the vanishing limit of the third moment of Maass-Hecke eigenforms on the modular surface $P S L(2, \mathbb{Z}) \backslash \mathbb{H}^{2}$ proved by Watson [42]. As for the even-order moments, Spinu [39] showed that the fourth moment of the $L^{2}$ normalized Eisenstein series on the modular surface is bounded. This last result is also remarkable in that it establishes for the continuous spectrum a special case of a still wide open conjecture originally formulated by Iwaniec and Sarnak [21] about the $L^{p}$-norm of the Laplace eigenfunctions: for any "nice enough" compact subset $K$ of a hyperbolic surface, $2<p \leq \infty$ and $\epsilon>0$, the $L^{2}$-normalized eigenfunction $\phi_{\lambda}$ corresponding to the eigenvalue $\lambda$ should satisfy $\left\|\phi_{\lambda}\right\|_{L^{p}(K)}=\mathcal{O}_{p, \epsilon, K}\left(\lambda^{\epsilon}\right)$; see $\S 4$ in [36] and references therein for more details and for other related results and conjectures.

In the general setting of compact Riemannian manifolds, one can mention the work of Canzani et al. [10] in which the authors study the distribution of the real part of perturbed 
eigenfunctions of the Schrödinger operator. In particular, they show that the odd-order moments vanish when the geodesic flow is ergodic, as predicted by Berry's conjecture.

On the other hand, the random wave model is known not to apply universally. For example, a consequence of such a model for the eigenfunctions $\phi_{\lambda}$ (of eigenvalues $\lambda$ ) would be that they satisfy

$$
\left\|\phi_{\lambda}\right\|_{\infty} \approx \sqrt{\log \lambda}
$$

in the high-energy limit; see, e.g., $\$ 1$ in [35]. However, Milićević showed in [28] that the high-energy Hecke-Maass eigenforms on arithmetic hyperbolic surfaces take values larger than what would be predicted by (1.2) if the Random Wave Conjecture were valid.

In the case at hand, one can easily check that the $L^{\infty}$-norm of $E\left(s_{\lambda} ; m, \xi\right)$ is bounded uniformly in the parameter $\lambda$; see $\S 2.2 .4$ and, in particular, Lemma 2.20 below. Consequently, it is not expected that the moments of the real and imaginary parts of the Eisenstein series on convex co-compact hyperbolic manifolds reflect the ones of the normal distribution.

\subsection{Presentation of the results}

Since

$$
F_{\lambda}(m, \xi)=\frac{i^{\frac{3}{2} \pm \frac{1}{2}}}{2}\left(E\left(s_{\lambda} ; m, \xi\right) \pm \overline{E\left(s_{\lambda} ; m, \xi\right)}\right)
$$

it follows from the binomial expansion that calculating the $p$-th moment of the real and imaginary parts of $E\left(s_{\lambda} ; m, \xi\right)$ at high-energy boils down to finding the limit as $\lambda \rightarrow \infty$ of the integrals

$$
I_{k}^{p}(\lambda, \xi):=\int_{X} a(m)\left(E\left(s_{\lambda} ; m, \xi\right)\right)^{k}\left(\overline{E\left(s_{\lambda} ; m, \xi\right)}\right)^{p-k} d v(m)
$$

for all integers $0 \leq k \leq p$. This thesis is then dedicated to studying the limit $\lambda \rightarrow \infty$ of integrals of the form (1.4). Notice that (1.1) corresponds to the special case where $p=2$ and $k=1$. In this sense, our objective can be seen as extending the result of Guillarmou and Naud stated above. 
The main result of this work is the following theorem, which describes the limit at highenergy of all the moments of the Eisenstein series when the dimension of the limit set is small enough. For more details about the notation introduced in the statement of this theorem as well as for definitions and elementary facts about integer and set partitions, the reader is urged to consult $§ 3.1$.

Theorem 1.1. Let $X=\Gamma \backslash \mathbb{H}^{n+1}$ be a convex co-compact hyperbolic manifold and assume that $\delta_{\Gamma}<n / 2$. Let $a \in C_{0}^{\infty}(X), \xi \in \partial \bar{X}$ and $I_{k}^{p}$ be defined by (1.4). Then for all integers $0 \leq k \leq p$,

$$
I_{k}^{p}(\lambda, \xi) \longrightarrow \begin{cases}0, & \text { if } k \neq \frac{p}{2} \\ \int_{X} a(m) f_{p}(m, \xi) d v_{X}(m), & \text { if } k=\frac{p}{2}\end{cases}
$$

as $\lambda \rightarrow \infty$, where

$$
f_{p}(m, \xi):=\sum_{l=1}^{p / 2} \sum_{\theta \in \Theta_{l}}\left(C_{\theta} \prod_{r=1}^{l} E\left(\theta_{r} n ; m, \xi\right)\right),
$$

with $\Theta_{l}$ the set of all length $l$ integer partitions $\theta=\left(\theta_{1}, \ldots, \theta_{l}\right)$ of $\frac{p}{2}$ and

$$
C_{\theta}=\sum_{\substack{\pi \in L_{p / 2}, \theta(\pi)=\theta}}\left(\begin{array}{c}
k \\
\theta(\pi)
\end{array}\right)+\sum_{\substack { \pi^{(s)} \\
\begin{subarray}{c}{\pi^{\prime}<\pi^{(1)}<\pi, \theta(\pi)=\theta{ \pi ^ { ( s ) } \\
\begin{subarray} { c } { \pi ^ { \prime } < \pi ^ { ( 1 ) } < \pi , \\
\theta ( \pi ) = \theta } }\end{subarray}}(-1)^{s}\left(\begin{array}{c}
k \\
\theta\left(\pi^{(s)}\right)
\end{array}\right),
$$

where $L_{p / 2}$ is the set of all partitions of a set of $p / 2$ elements and the second sum is over all chains (if any) of set partitions $\pi^{(s)}<\cdots<\pi^{(1)}<\pi$ of any length $s+1$ satisfying the only condition that their underlying integer partition $\theta(\pi)$ is equal to $\theta$.

Remark 1.1. More explicit expressions of $f_{p}$ are given for $p=2,4,6$ and 8 in Chapter 3. (See (3.9), (3.12), (3.19) and (3.20) respectively.)

Remark 1.2. The moments of $F_{\lambda}$ at high-energy is a direct corollary of Theorem 1.1. See $\S 3.3$ below for a precise statement.

In the rest of the thesis, we are interested in the question of the rate of convergence of $I_{k}^{p}(\lambda, \xi)$ to the limits given by $(1.5)$ as $\lambda \rightarrow \infty$. In the case where $p$ is odd, a general argument allowed us to find an error term for any $k$ and on manifolds of any dimension. 
Theorem 1.2. Let $X=\Gamma \backslash \mathbb{H}^{n+1}$ be a convex co-compact hyperbolic manifold and assume that $\delta_{\Gamma}<n / 2$. Let $p \geq 1$ be odd. Let $a \in C_{0}^{\infty}(X), \xi \in \partial \bar{X}$ and $I_{k}^{p}$ be defined by (1.4). Then for all $0 \leq k \leq p$,

$$
I_{k}^{p}(\lambda, \xi)=\mathcal{O}\left(\lambda^{-\frac{1}{d}}\right)
$$

as $\lambda \rightarrow \infty$, where $d=4 p$ if $k=0$ or $p$ and $d=4 p+2$ if $1 \leq k \leq p-1$. Moreover, the implied constant in the bound $\mathcal{O}\left(\lambda^{-\frac{1}{d}}\right)$ is independent of $\xi \in \partial \bar{X}$.

Remark 1.3. The bounds given by this theorem are not sharp in general. As attests Proposition 4.1 below, a faster convergence can be achieved on surfaces when $p=3$. The arguments leading to Theorem 1.2 do not essentially depend on the power $p$ (as long as it is odd) or on the dimension of the manifold. The cost of such a general proof is that it relies on fairly rough estimates. For this reason, we believe that sharper bounds can be obtained for any value of $p$ and $n$.

For $p$ even, the "middle term", i.e., $I_{p / 2}^{p}(\lambda, \xi)$, corresponds to a restricted $L^{p}$-norm of the Eisenstein series to compact subsets of the manifold. Therefore, this quantity is non-negative for any $\lambda$. Moreover, since the Eisenstein series do not vanish almost everywhere in the limit $\lambda \rightarrow \infty$, the limit of $I_{p / 2}^{p}(\lambda, \xi)$ as $\lambda \rightarrow \infty$ should always be positive. It follows in particular that the functions $f_{p}(m, \xi)$ defined by (1.6) and (1.7) in the statement of Theorem 1.1 are non-negative.

The restricted $L^{2}$-norm (1.1) calculated by Guillarmou and Naud gives also an indication of the rate of convergence. For higher values of $p$ even, we were not able to obtain a general result like Theorem 1.2. The technique employed to approach the odd powers turned out to be ineffective to estimate the rate of convergence in the case where $p$ is even. Restricting ourselves to surfaces, we managed to determine an error term for the restricted $L^{4}$-norm. 
Theorem 1.3. Let $X=\Gamma \backslash \mathbb{H}^{2}$ be a convex co-compact hyperbolic surface and assume that $\delta_{\Gamma}<1 / 2$. Let $a \in C_{0}^{\infty}(X), \xi \in \partial \bar{X}$ and $I_{k}^{p}$ be defined by (1.4). Then

$$
I_{2}^{4}(\lambda, \xi)=\int_{X} a(m) f_{4}(m, \xi) d v_{X}(m)+\mathcal{O}\left(\lambda^{\delta_{\Gamma}-\frac{1}{2}}\right)
$$

as $\lambda \rightarrow \infty$, where $f_{4}(m, \xi):=2(E(1 ; m, \xi))^{2}-E(2 ; m, \xi)$. Moreover, the implied constant in the bound $\mathcal{O}\left(\lambda^{\delta_{\Gamma}-\frac{1}{2}}\right)$ is independent of $\xi \in \partial \bar{X}$.

Using the right-hand side of (1.1) as a $L^{2}$-normalization factor, this theorem can be seen as a special case of the $L^{4}$-norm problem on hyperbolic surface as well as an analogue of Spinu's result described above.

Theorem 1.2 gives us readily a remainder for all the odd-order moments of the real and imaginary parts of $E$.

Corollary 1.4. Under the hypotheses of Theorem 1.2,

$$
\int_{X} a(m)\left(F_{\lambda}(m, \xi)\right)^{p} d v_{X}(m)=\mathcal{O}\left(\lambda^{-\frac{1}{4 p+2}}\right) .
$$

as $\lambda \rightarrow \infty$, where the implied constant is independent of $\xi \in \partial \bar{X}$.

The error terms for the second and the fourth moments of $F_{\lambda}$ are essentially given by (1.1) and by Theorem 1.3 respectively. However, it remains to find the rate of convergence of the "cross terms" in the binomial expansion of $F_{\lambda}$, i.e., all the terms of the form (1.4) with $k \neq p / 2$, which, as we already know from Theorem 1.1, vanish as $\lambda \rightarrow \infty$. For $p=2$, this is done at the end of $\S 5.3$ :

$$
\int_{X} a(m)\left(F_{\lambda}\left(s_{\lambda} ; m, \xi\right)\right)^{2} d v_{X}(m)=\frac{1}{2} \int_{X} a(m) E(n ; m, \xi) d v_{X}(m)+\mathcal{O}\left(\lambda^{-\min \left\{n-2 \delta_{\Gamma}, \frac{1}{8}\right\}}\right) .
$$

as $\lambda \rightarrow \infty$. The fourth moment of $F_{\lambda}$ is the content of the following corollary.

Corollary 1.5. Under the hypotheses of Theorem 1.3,

$$
\int_{X} a(m)\left(F_{\lambda}(m, \xi)\right)^{4} d v_{X}(m)=\frac{3}{8} \int_{X} a(m) f_{4}(m, \xi) d v_{X}(m)+\mathcal{O}\left(\lambda^{-\min \left\{\frac{1}{2}-\delta_{\Gamma}, \frac{1}{18}\right\}}\right)
$$

as $\lambda \rightarrow \infty$, where the implied constant is independent of $\xi \in \partial \bar{X}$. 
Remark 1.4. The fact that the cross terms vanish polynomially for $p=2$ in general and for $p=4$ when $X$ is a surface (see Proposition 5.3 below and the discussion following it) leads us to believe that Theorem 1.2 could remain valid for any $p$ even, when $k \neq p / 2$. However, as will be seen in Chapter 4 below, the proof of this theorem does not allow us to draw such a conclusion.

Although the random wave model cannot be expected to describe the behaviour of $F_{\lambda}$ at high-energy, the vanishing of the odd-order moments exhibited by Corollary 1.4 is in agreement with the vanishing of the odd-order moments of the normal distribution predicted by Berry. In particular, a probability law corresponding to the moments of $F_{\lambda}$ would have to be symmetric.

\subsection{Organization of the thesis}

Chapter 2 constitutes a brief account of the background material on which the rest of the thesis is based. Among other things, precise definitions of the less common mathematical concepts mentioned in the introduction are given there. Our treatment in this chapter is essentially expository, though some (elementary) non-standard results will be proved as well.

Our presentation of the proofs of the main theorems, stated in $\S 1.2$, begins in Chapter 3. The proof of Theorem 1.1 is contained entirely in $\S 3.1$. In $\S 3.2$, we explain how to evaluate explicitly the coefficients $C_{\theta}$ 's (see (1.7) above) appearing in the limits provided by this theorem. Moreover, a statement similar to the ones of Corollary 1.4 and Corollary 1.5 about the high-energy moments of the real and imaginary parts of Eisenstein series is stated in $\S 3.3$.

The next two chapters revolve around studying the rate of convergence of the limits found previously. Chapter 4 is devoted to the odd-order moments. In addition to the proof of Theorem 1.2, the reader will find there a treatment of the special case where $p=3$ and $n=1$, for which an improved remainder term can be obtained. Besides providing a sharper bound for the third moment, the proof of this result introduces the basis of a method which will prove useful for studying the fourth moment on surfaces in the following chapter. 
In Chapter 5, we are concerned with the special case where $n=1$ and $p=4$. For the sake of completeness and as a preliminary for the proof of Theorem 1.3, the argument of Guillarmou and Naud which led to (1.1) will be recalled at the beginning of this chapter. We prove Theorem 1.3 in $§ 5.2$. Finally, Corollary 1.5 and equation (1.8) are dealt with in $\S 5.3$.

We conclude this thesis in Chapter 6 with a brief discussion about the limits of our approach in determining the high-energy asymptotics of the moments of Eisenstein series. Moreover, we suggest there some directions for further research.

\section{Contributions of the author}

Unless explicitly stated otherwise, all the results proved in this thesis are the work of the author alone. In particular, to the best of the author's knowledge, all the theorems and corollaries stated in $\S 1.2$ and proved in Chapter 3, Chapter 4, $\S 5.2$ and $\S 5.3$ are original contributions to knowledge. 


\section{CHAPTER 2 Preliminaries}

The objective of this chapter is twofold. Firstly, it serves as an exposition of important concepts originating from the geometry and spectral theory of hyperbolic manifolds. Our treatment is, however, by no means exhaustive, nor should it be considered as a complete introduction to these topics. We made an effort to restrict the presentation to the definitions and facts that are, in our opinion, essential for understanding the rest of the thesis. The reader interested in a thorough treatment of hyperbolic manifolds should consult, for

example, [34]. For a unified exposition of the spectral theory of infinite-area surfaces, the reader is referred to [7].

Secondly, this chapter presents some preliminary results which, though elementary, will play an important role in the proof of the main theorems. They are spread throughout the different sections, according to their content. Proofs are generally given for facts that are not standard.

\subsection{Hyperbolic manifolds}

For the rest of this thesis, the term hyperbolic manifold will refer to a complete connected orientable Riemannian manifold of constant sectional curvature -1 . It is a basic fact of Riemannian geometry that there exists up to isometry a unique $(n+1)$-dimensional simplyconnected hyperbolic manifold, the hyperbolic $(n+1)$-space $\mathbb{H}^{n+1}$. In order to do calculations on this space, one has to choose a model of hyperbolic space, i.e., an isometric copy of $\mathbb{H}^{n+1}$. The model that is the best suited for our purposes is defined as follows. Let

$$
\mathbb{B}^{n+1}:=B_{0}^{n+1}(1)=\left\{x=\left(x_{1}, x_{2}, \ldots, x_{n+1}\right) \in \mathbb{R}^{n+1}:|x|^{2}=\sum_{i=1}^{n+1} x_{i}^{2}<1\right\} .
$$


One can check that endowed with the metric tensor

$$
d s^{2}=\frac{4 \sum_{1}^{n+1} d x_{i}^{2}}{\left(1-|x|^{2}\right)^{2}},
$$

the open unit ball $\mathbb{B}^{n+1}$ becomes a hyperbolic manifold, which usually takes the name of the Poincaré ball model. Since this model is the only one that will be used in this text, the term hyperbolic space and the symbol $\mathbb{H}^{n+1}$ will also refer to the manifold $\mathbb{B}^{n+1}$. It is important to notice that under the Poincaré ball model, the geodesics correspond to the arcs of circles which intersect perpendicularly the spherical boundary $S^{n}:=\partial \mathbb{B}^{n+1}$ of $\mathbb{B}^{n+1}$.

The Riemannian metric $(2.1)$ induces a distance function $d(\cdot, \cdot)$ on $\mathbb{B}^{n+1}$, which is given (see, e.g., Theorem 4.5 .1 in [34]) by

$$
\cosh d(x, y)=1+\frac{2|x-y|^{2}}{\left(1-|x|^{2}\right)\left(1-|y|^{2}\right)},
$$

for any $x, y \in \mathbb{B}^{n+1}$.

Let $\operatorname{Isom}_{+}\left(\mathbb{H}^{n+1}\right)$ be the group of orientation-preserving isometries of $\mathbb{H}^{n+1}$ and let $\Gamma$ be a torsion-free discrete subgroup of $\operatorname{Isom}_{+}\left(\mathbb{H}^{n+1}\right)$. It follows from elementary Riemannian geometry that the quotient $\Gamma \backslash \mathbb{H}^{n+1}$ is a complete Riemannian manifold with the natural hyperbolic metric inherited from $\mathbb{H}^{n+1}$. On the other hand, it is known since the time of Hopf that any hyperbolic manifold is of this form. (See, e.g., Theorem 8.5.9 in [34] for a proof of this classic result.) Therefore, in what follows, any hyperbolic manifold $M$ will be associated with a discrete group $\Gamma$ of orientation-preserving isometries of $\mathbb{H}^{n+1}$ and the symbols $M$ and $\Gamma \backslash \mathbb{H}^{n+1}$ will be used interchangeably to refer to this hyperbolic manifold.

\subsubsection{Möbius transformations}

As noted above, the group $\operatorname{Isom}_{+}\left(\mathbb{H}^{n+1}\right)$ plays a fundamental role in the theory of hyperbolic manifolds. In this subsection, we will describe the structure of this group by giving a characterization of it in terms of Möbius transformations. 
Let $S(a, r):=S^{n}(a, r)$ be the sphere of radius $r$ centered at $a$ in $\mathbb{R}^{n+1}$, i.e.,

$$
S(a, r):=\left\{x \in \mathbb{R}^{n+1}:|x-a|=r\right\}
$$

We define the reflection in the sphere $S(a, r)$ as the function

$$
\sigma(x):=a+\left(\frac{r}{|x-a|}\right)^{2}(x-a), \quad x \in \mathbb{R}^{n+1}-\{a\} .
$$

The definition of this function can be extended to $\mathbb{R}^{n+1}$ by adding the point $\infty$ and by defining $\sigma(a):=\infty$. On the other hand, by defining $\sigma(\infty):=a$, the function $\sigma$ is now defined in all of $\mathbb{R}^{n+1} \cup\{\infty\}$.

Similarly, for any (extended) plane

$$
P(a, t):=\left\{x \in \mathbb{R}^{n+1}: a \cdot x=t\right\} \cup\{\infty\}, \quad a \in \mathbb{R}^{n+1}-\{0\}, t \in \mathbb{R},
$$

we can define the reflection in the plane $P(a, t)$ for all $x \in \mathbb{R}^{n+1} \cup\{\infty\}$ by

$$
\sigma(x):= \begin{cases}x-2(x \cdot a-t) \frac{a}{|a|^{2}}, & \text { if } x \in \mathbb{R}^{n} \\ \infty, & \text { if } x=\infty\end{cases}
$$

A function $\gamma: \mathbb{R}^{n+1} \cup\{\infty\} \rightarrow \mathbb{R}^{n+1} \cup\{\infty\}$ is said to be a Möbius transformation of $\mathbb{B}^{n+1}$ if it is a finite composition of reflections (in spheres or planes) that leaves $\mathbb{B}^{n+1}$ invariant. We will write $M\left(\mathbb{B}^{n+1}\right)$ for the set of all Möbius transformations of $\mathbb{B}^{n+1}$ which are also orientation-preserving.

The following theorem, in conjunction with the remarks made at the beginning of this section, establishes the connection between Möbius transformations and hyperbolic manifolds.

Theorem 2.1. The groups $\operatorname{Isom}_{+}\left(\mathbb{H}^{n+1}\right)$ and $M\left(\mathbb{B}^{n+1}\right)$ are isomorphic.

For a proof of this proposition, the reader is referred to $\S 4.5$ in [34]. (See, in particular, Theorem 4.5.2 and Corollary 1 of this section.) In view of Theorem 2.1, from now on, we will make no difference between the elements of $\operatorname{Isom}_{+}\left(\mathbb{H}^{n+1}\right)$ and orientation-preserving Möbius 
transformations of $\mathbb{B}^{n+1}$. The following proposition gives a characterization of the Möbius transformations which will prove useful in the calculations to come.

Proposition 2.2. Let $\gamma$ be a Möbius transformation of $\mathbb{B}^{n+1}$.

- If $\gamma(\infty)=\infty$, then $\gamma$ is an orthogonal transformation.

- If $\gamma(\infty) \neq \infty$, then there exists an orthogonal transformation $\psi$ such that

$$
\gamma(x)=\psi \circ \sigma(x), \quad x \in \mathbb{R}^{n+1} \cup\{\infty\}
$$

where $\sigma$ is the reflection in the sphere $S(a, r)$ with

$$
a=\gamma^{-1}(\infty) \quad \text { and } \quad r=\frac{1}{\sinh \left(\frac{1}{2} d(0, \gamma 0)\right)}
$$

Proof. See the proof of Theorem 4.4.7 in [34] and also $§ 3.5$ in [4].

Any Möbius transformation other than the identity can fix precisely zero, one or two points in $S^{n}$. (See $\S 4.7$ in [34] for more details about this fact.) This justifies the use of the following classification. We will say that a Möbius transformation is

- elliptic if it fixes no point of $S^{n}$;

- parabolic if it fixes exactly one point of $S^{n}$;

- hyperbolic (or loxodromic) if it fixes exactly two points of $S^{n}$.

Moreover, it is a well-known fact that elliptic transformations fix exactly one point of $\mathbb{B}^{n+1}$ whereas parabolic and hyperbolic transformations do not fix any point of $\mathbb{B}^{n+1}$. It follows then from the remarks at the beginning of this section that in order for $\Gamma \backslash \mathbb{H}^{n+1}$ to be a manifold, the group $\Gamma$ must not contain any elliptic element.

The fixed point $\xi \in S^{n}$ of a parabolic transformation $\gamma$ is always attractive, in the sense that for any $x \in \overline{\mathbb{B}^{n+1}}$, we have $\gamma^{j} x \rightarrow \xi$ as $j \rightarrow \infty$. On the other hand, if $\gamma$ is hyperbolic, then one (and, of course, only one) of its two fixed points is attractive. The other one is then said to be repulsive. It is clear, in this case, that the fixed points of $\gamma^{-1}$ are the same as the ones of $\gamma$, with the only difference that the attractive point of $\gamma$ is the repulsive point of $\gamma^{-1}$, and vice versa. (See $\S 4.7$ in [34] for more details.) 
We conclude this subsection with the statement and the proof of some elementary facts about isometries of $\mathbb{H}^{n+1}$ which will be used in the proofs presented in the subsequent chapters. The first of these results consists of two elementary and well-known formulae, the proof of which can be found in $§ 3.4$ and $\S 3.5$ of [4].

Proposition 2.3. Let $\gamma \in \operatorname{Isom}_{+}\left(\mathbb{H}^{n+1}\right)$ and suppose that $a_{\gamma}:=\gamma^{-1}(\infty) \neq \infty$. Then

$$
\sinh ^{2}\left(\frac{1}{2} d(0, \gamma 0)\right)=\frac{1}{\left|m-a_{\gamma}\right|\left|\gamma m-a_{\gamma}\right|}
$$

and

$$
\left|\gamma m-\gamma m^{\prime}\right|=\frac{\left|m-m^{\prime}\right|}{\sinh ^{2}\left(\frac{1}{2} d(0, \gamma 0)\right)\left|m-a_{\gamma}\right|\left|m^{\prime}-a_{\gamma}\right|},
$$

for all $m, m^{\prime} \in \overline{\mathbb{B}^{n+1}}$.

The next two results are elementary as well. However, since they do not appear in any reference known to the author, they will be stated with a proof.

Lemma 2.4. Let $\gamma \in \operatorname{Isom}_{+}\left(\mathbb{H}^{n+1}\right)$ and suppose that $a_{\gamma}:=\gamma^{-1}(\infty) \neq \infty$. Let $D_{m} \gamma(m)$ denote the differential of $\gamma$ at the point $m \in \mathbb{H}^{n+1}$. If $\lambda$ is an eigenvalue of $D_{m} \gamma(m)$, then

$$
|\lambda|=\left|\operatorname{det}\left(D_{m} \gamma(m)\right)\right|^{\frac{1}{n+1}}=\frac{1-|\gamma m|^{2}}{1-|m|^{2}}=\frac{1}{\sinh ^{2}\left(\frac{1}{2} d(0, \gamma 0)\right)\left|a_{\gamma}-m\right|^{2}}=\left|D_{m} \gamma(m)\right| \text {. }
$$

Moreover,

$$
\left|\left(D_{m} \gamma(m)\right) v\right|=|\lambda||v|
$$

for all $v \in T_{m} \mathbb{H}^{n+1} \cong \mathbb{R}^{n+1}$.

Proof. Let $\gamma \in \operatorname{Isom}_{+}\left(\mathbb{H}^{n+1}\right)$. Let $\langle\cdot, \cdot\rangle$ denote the standard dot product in $\mathbb{R}^{n+1}$. By (2.1), the inner product $\langle\cdot, \cdot\rangle_{m}$ on $T_{m} \mathbb{H}^{n+1}$ induced by the hyperbolic metric satisfies

$$
\langle\cdot, \cdot\rangle_{m}=\frac{4}{\left(1-|m|^{2}\right)^{2}}\langle\cdot, \cdot\rangle
$$


Since $\gamma$ is an isometry, for all $m \in \mathbb{H}^{n+1}$ and all $v \in T_{m} \mathbb{H}^{n+1} \cong \mathbb{R}^{n+1}$, we have

$$
\begin{aligned}
\frac{4}{\left(1-|\gamma m|^{2}\right)^{2}}\left\langle\left(D_{m} \gamma(m)\right) v,\left(D_{m} \gamma(m)\right) v\right\rangle & =\left\langle\left(D_{m} \gamma(m)\right) v,\left(D_{m} \gamma(m)\right) v\right\rangle_{\gamma(m)} \\
& =\langle v, v\rangle_{m}=\frac{4}{\left(1-|m|^{2}\right)^{2}}\langle v, v\rangle .
\end{aligned}
$$

or, equivalently,

$$
\left\langle\frac{1-|m|^{2}}{1-|\gamma m|^{2}}\left(D_{m} \gamma(m)\right) v, \frac{1-|m|^{2}}{1-|\gamma m|^{2}}\left(D_{m} \gamma(m)\right) v\right\rangle=\langle v, v\rangle \text {. }
$$

It follows that for any $m \in \mathbb{H}^{n+1}$,

$$
\frac{1-|m|^{2}}{1-|\gamma m|^{2}}\left(D_{m} \gamma(m)\right)
$$

is an orthogonal transformation of $\mathbb{R}^{n+1}$, from which we conclude that if $\lambda$ is an eigenvalue of $D_{m} \gamma(m)$, then

$$
|\lambda|=\frac{1-|\gamma m|^{2}}{1-|m|^{2}}, \quad \text { and } \quad\left|\operatorname{det}\left(D_{m} \gamma(m)\right)\right|^{\frac{1}{n+1}}=\left(\prod_{\lambda \text { e.v. }}|\lambda|\right)^{\frac{1}{n+1}}=\frac{1-|\gamma m|^{2}}{1-|m|^{2}}
$$

The remaining equalities are standard elementary facts; see, e.g., $§ 3.4$ and $\S 3.5$ in [4]. The last part of the statement is a direct consequence of (2.6).

Here, and in the rest of the text, we will use the following notation for the partial derivatives of a function. If $\alpha \in \mathbb{N}_{0}^{n+1}$ and $|\alpha|=\sum_{k=1}^{n+1} \alpha_{k}$, then

$$
\partial_{\alpha} f(x)=\frac{\partial^{|\alpha|} f(x)}{\partial x_{1}^{\alpha_{1}} \cdots \partial x_{n+1}^{\alpha_{n+1}}}
$$

for any $f \in C^{|\alpha|}\left(\mathbb{R}^{n+1}\right)$.

Lemma 2.5. Let $F \in C^{\infty}\left(\mathbb{H}^{n+1}\right)$ and $K$ be a compact subset of $\mathbb{B}^{n+1}$. Then for any $\gamma \in$ $\operatorname{Isom}_{+}\left(\mathbb{H}^{n+1}\right)$, we have

$$
\left|\partial_{\alpha} F\left(\gamma^{-1} m\right)\right| \leq \frac{C_{\alpha} \max _{|\beta| \leq|\alpha|} \max _{m_{0} \in K} \partial_{\beta} F\left(m_{0}\right) .}{\left(1-|m|^{2}\right)^{|\alpha|}}, \quad \alpha \in \mathbb{N}_{0}^{n+1}, \quad m \in \gamma K
$$

for some $C_{\alpha}>0$ depending only on $F, K$ and $\alpha$. 
Proof. By the higher chain formula proved in [26], we know that $\partial_{\alpha} F\left(\gamma^{-1} m\right)$ is a sum of terms of the form

$$
\left.\left.C_{\beta} \frac{\partial^{|\beta|} F(m)}{\partial x_{1}^{\beta_{1}} \cdots \partial x_{n+1}^{\beta_{n+1}}}\right|_{m=\gamma^{-1} m_{0}} \cdot \prod_{k=1}^{s} \frac{\partial^{\left|r_{k}\right|}\left(\gamma^{-1} m\right)_{j}}{\partial x_{1}^{r_{k 1}} \cdots \partial x_{n+1}^{r_{k(n+1)}}}\right|_{m=m_{0}}
$$

for some index $\beta$ such that $|\beta| \leq|\alpha|$ and for some $s$ and indices $r_{k}$ 's satisfying $\sum_{k=1}^{s}\left|r_{k}\right|=|\alpha|$. Observe that under this notation, the $r_{k}$ 's are not necessarily distinct. For $m_{0}=\gamma m \in \gamma K$, the point $\gamma^{-1} m_{0}$ varies over a compact subset of $\mathbb{B}^{n+1}$. Hence,

$$
\left|\frac{\partial^{|\beta|} F(m)}{\partial x_{1}^{\beta_{1}} \cdots \partial x_{n+1}^{\beta_{n+1}}}\right|_{m=\gamma^{-1} m_{0}} \leq C_{\beta}^{\prime} \max _{m \in K} \partial_{\beta} F(m)
$$

uniformly in $\gamma$. If $\gamma^{-1}(\infty)=\infty$, then $\gamma$ is a rotation and the product term in (2.8) can also be bounded by a constant. Since, moreover, $\gamma K$ is contained in a compact subset of $\mathbb{H}^{n+1}$ uniformly for any rotation $\gamma$, the statement of the lemma follows trivially, granted that the constant $C_{\alpha}$ is chosen large enough.

So, from now on, we suppose that $\gamma^{-1}(\infty) \neq \infty$. By (2.3) and Proposition 2.2, we have

$$
\psi\left(\gamma^{-1} m\right)=a_{\gamma^{-1}}+\frac{m-a_{\gamma^{-1}}}{\sinh ^{2}\left(\frac{1}{2} d\left(0, \gamma^{-1} 0\right)\right)\left|m-a_{\gamma^{-1}}\right|^{2}},
$$

for some orthogonal transformation $\psi$. It follows that

$$
\left|\partial_{r_{k}}\left(\gamma^{-1} m\right)\right| \leq \frac{C_{r_{k}}}{\sinh ^{2}\left(\frac{1}{2} d\left(0, \gamma^{-1} 0\right)\right)\left|m-a_{\gamma^{-1}}\right| r_{k} \mid+1} .
$$

Moreover, by equation (2.4) of Proposition 2.3,

$$
\sinh ^{2}\left(\frac{1}{2} d\left(0, \gamma^{-1} 0\right)\right)=\frac{1}{\left|m-a_{\gamma^{-1}}\right|\left|\gamma^{-1} m-a_{\gamma^{-1}}\right|}
$$

Therefore, combining the last two equations and Lemma 2.4, and using again the compactness of $K$, we get

$$
\left|\partial_{r_{k}}\left(\gamma^{-1} m\right)\right| \leq \frac{C_{r_{k}}\left|\gamma^{-1} m-a_{\gamma^{-1}}\right|}{\left|m-a_{\gamma^{-1}}\right|^{\left|r_{k}\right|}}=\frac{C_{r_{k}}\left(1-\left|\gamma^{-1} m\right|^{2}\right)^{\left|r_{k}\right|}}{\left|\gamma^{-1} m-a_{\gamma^{-1}}\right|^{r_{k}-1}\left(1-|m|^{2}\right)^{\left|r_{k}\right|}} \leq \frac{C_{r_{k}}^{\prime}}{\left(1-|m|^{2}\right)^{\left|r_{k}\right|}}
$$

The conclusion of the lemma follows then from (2.8), (2.9) and (2.10). 


\subsubsection{Limit set}

Let $\Gamma \leq \operatorname{Isom}_{+}\left(\mathbb{H}^{n+1}\right)$. A point $\xi \in S^{n}$ is said to be a limit point of $\Gamma$ if there exists a sequence $\left\{\gamma_{j}\right\}_{j=1}^{\infty} \subset \Gamma$ such that $\gamma_{j} x \rightarrow \xi$ as $j \rightarrow \infty$ for some $x \in \mathbb{H}^{n+1}$. Notice that the definition above is left unchanged if we replace the words "for some" by "for any". Indeed, fix $x_{0} \in \mathbb{H}^{n+1}$ and suppose that $\gamma_{j} x \rightarrow \xi$. Since $\gamma_{j}$ is an isometry, we have $d\left(\gamma_{j} x, \gamma_{j} x_{0}\right)=d\left(x, x_{0}\right)$. It follows from (2.2) that the Euclidean distance in $\mathbb{B}^{n+1}$ between $\gamma_{j} x$ and $\gamma_{j} x_{0}$ tends to zero as $j \rightarrow \infty$. In other words, we have $\gamma_{j} x_{0} \rightarrow \xi$, as required.

The set $\Lambda_{\Gamma}:=\Gamma x_{0}$ (for any $x_{0} \in \mathbb{H}^{n+1}$ ) of all limit points of $\Gamma$ is called the limit set of $\Gamma$. The complement of the limit set of $\Gamma$ in $S^{n}$ is called the set of discontinuity of $\Gamma$, and we write $\Omega_{\Gamma}:=S^{n}-\Lambda_{\Gamma}$.

Proposition 2.6. If $\xi \in \Omega_{\Gamma}$, then $\xi$ is not fixed by any element of $\Gamma$ other than the identity.

Proof. This is a direct consequence of the fact that if $\xi$ is the fixed point of some parabolic or hyperbolic transformation, then it is the attractive fixed point of some transformation $\gamma$ (see the previous section), i.e., for any $x \in \overline{\mathbb{B}^{n+1}}$, we have $\gamma^{j} x \rightarrow \xi$ as $j \rightarrow \infty$. Hence $\xi \notin \Omega_{\Gamma}$.

We will classify the discrete groups of hyperbolic isometries according to the "number of points" of their limit set. If $\Lambda_{\Gamma}=S^{n+1}$, then $\Gamma$ is said to be of the first kind. Otherwise, the discrete group $\Gamma$ is said to be of the second kind. The following theorem imposes restrictions on the possible types of limit sets for a group of the second kind.

Theorem 2.7. Let $\Gamma \leq \operatorname{Isom}_{+}\left(\mathbb{H}^{n+1}\right)$ be a discrete group of the second kind. If $\Lambda_{\Gamma}$ is finite, then it contains exactly zero, one or two points. Otherwise, $\Lambda_{\Gamma}$ is a perfect, nowhere dense subset of $S^{n}$.

Proof. This statement is standard. It follows, for example, from Theorem 12.2.1 and Theorem 12.2.5 in [34]. 
This result motivates the further classification of the discrete groups of hyperbolic isometries. The group $\Gamma$ (resp. the hyperbolic manifold $\Gamma \backslash \mathbb{H}^{n+1}$ ) is said to be elementary if $\Lambda_{\Gamma}$ is finite.

Theorem 2.7 shows us that the limit set of any non-elementary discrete group of the second kind is a "Cantor type set". It then makes sense to consider its Hausdorff dimension. If we define

$$
\delta_{\Gamma}:=\operatorname{dim}_{\text {Haus }}\left(\Lambda_{\Gamma}\right),
$$

then it follows directly from Theorem 2.7 and from the definitions above that

- $\delta_{\Gamma}=0$ if $\Gamma$ is elementary;

- $0<\delta_{\Gamma}<n$ if $\Gamma$ is non-elementary of the second kind;

- $\delta_{\Gamma}=n$ if $\Gamma$ is of the first kind.

For any discrete subgroup $\Gamma \leq \operatorname{Isom}_{+}\left(\mathbb{H}^{n+1}\right)$, one can consider the intersection of all convex subsets (in the hyperbolic sense) of $\overline{\mathbb{B}^{n+1}}$ that contain $\Lambda_{\Gamma}$. The resulting set will be called the (hyperbolic) convex hull of $\Lambda_{\Gamma}$ and will be denoted by $C(\Gamma)$. It can be shown that $C(\Gamma) \cap \mathbb{B}^{n+1}$ is a closed, convex, $\Gamma$-invariant subset of $\mathbb{B}^{n+1}$. Therefore, it makes sense to consider its quotient by $\Gamma$,

$$
C(X):=\Gamma \backslash\left(C(\Gamma) \cap \mathbb{B}^{n+1}\right),
$$

which will be referred to as the convex core of $X=\Gamma \backslash \mathbb{H}^{n+1}$.

\subsubsection{Geometrically finite manifolds}

In this subsection, we will introduce a class of hyperbolic manifolds that have a relatively simple geometry. We will restrict ourselves to these manifolds in the rest of the text. Their definition depends on the following concept.

Let $\Gamma \leq \operatorname{Isom}_{+}\left(\mathbb{H}^{n+1}\right)$. A connected subset $\mathcal{F}$ of $\mathbb{B}^{n+1}$ is said to be a fundamental domain for $\Gamma$ if the three following conditions are satisfied:

1. $\mathcal{F}$ is open in $\mathbb{B}^{n+1}$; 
2. For all $\gamma \neq \gamma^{\prime} \in \Gamma$, we have $\gamma \mathcal{F} \cap \gamma^{\prime} \mathcal{F}=\emptyset$;

3. $\mathbb{B}^{n+1}=\bigcup_{\gamma \in \Gamma} \gamma \overline{\mathcal{F}}$.

It follows from this definition that the action of a group on its fundamental domain gives us a tessellation of $\mathbb{H}^{n+1}$.

A discrete subgroup $\Gamma$ of $\operatorname{Isom}_{+}\left(\mathbb{H}^{n+1}\right)$ (resp. a hyperbolic manifold $\Gamma \backslash \mathbb{H}^{n+1}$ ) is said to be geometrically finite if $\Gamma$ admits as a fundamental domain a finite-sided, convex geodesic polyhedron $\mathcal{F}$, every side $S$ of which satisfies $S=\mathcal{F} \cap \gamma \mathcal{F}$ for some $\gamma \in \Gamma$. Here, the term convex is used in the hyperbolic sense, meaning that for each pair of distinct points $x, y \in \mathcal{F}$, the geodesic segment joining $x$ and $y$ is contained in $\mathcal{F}$.

With the additional assumption that the discrete group $\Gamma$ is geometrically finite, we have the following characterization of the "kind" of $\Gamma$ according to the volume of its associated hyperbolic manifold $\Gamma \backslash \mathbb{H}^{n+1}$.

Theorem 2.8. Let $\Gamma$ be a discrete subgroup of $\operatorname{Isom}_{+}\left(\mathbb{H}^{n+1}\right)$. If $\Gamma$ is geometrically finite, then the volume of $\Gamma \backslash \mathbb{H}^{n+1}$ is finite if and only if $\Gamma$ is of the first kind.

Proof. This result is classic and follows, for example, from Theorem 12.2.13 and Theorem 12.4.8 in [34].

If $\mathcal{F}$ is the fundamental domain of some geometrically finite group $\Gamma$, then the fixed points of $\Gamma$ which are in the closure of $\mathcal{F}$, i.e., $\overline{\mathcal{F}} \cap \Lambda_{\Gamma}$, are all of the same type. More precisely, they are all fixed by a parabolic transformation of $\Gamma$. (See, e.g., Theorem 12.4.4 in [34].) Moreover, any given fundamental domain has only finitely many such points. (See, e.g., Theorem 12.4.1 in [34].) Every point of $\overline{\mathcal{F}} \cap \Lambda_{\Gamma}$ will be called a cusp point of $\mathcal{F}$ and the set $\overline{\mathcal{F}} \cap \Lambda_{\Gamma}$ will be referred to as the set of cusp points of $\mathcal{F}$ or a set of inequivalent cusp points for $\Gamma$.

If $c$ is a cusp point, then there exists a horosphere based at $c$ (i.e., an Euclidean sphere in $\overline{\mathbb{B}^{n+1}}$ tangent at $c$ ) such that its interior $B(c)$ has the property that

$$
B(c) \cap \gamma B(c), \quad \gamma \in \Gamma-\Gamma_{c},
$$


where $\Gamma_{c}$ is the stabilizer of $c$ in $\Gamma$. If $\pi: \mathbb{B}^{n+1} \rightarrow \Gamma \backslash \mathbb{B}^{n+1}$ is the quotient projection map, then the set $\pi(B(c))$ is called a cusp of $X:=\Gamma \backslash \mathbb{H}^{n+1}$. Moreover, the volume of $\pi(B(c))$ is finite.

In the geometrically finite case, the convex core of a manifold $M$ has the property that it becomes compact if we "remove" the cusps.

Theorem 2.9. If $X=\Gamma \backslash \mathbb{H}^{n+1}$ is a geometrically finite hyperbolic manifold, then $X$ has a finite number (possibly zero) of cusps $V_{1}, \ldots, V_{k}$, satisfying $\overline{V_{i}} \cap \overline{V_{j}}=\emptyset$ whenever $i \neq j$, such that $C(X)-\bigcup_{i=1}^{k} V_{i}$ is compact.

Proof. See the proof of Theorem 12.4.5 in [34].

For more information about cusps and the geometry of geometrically finite hyperbolic manifolds in general, the reader is encouraged to consult Chapter 12 in [34].

\subsubsection{Convex co-compact manifolds}

We conclude this section with the introduction of a class of manifolds which will be of great interest for us in the subsequent chapters. These geometrically finite manifolds can be characterized by the property that they don't have any cusp, which simplifies their analysis.

Let $\Gamma$ be a geometrically finite discrete subgroup of $\operatorname{Isom}_{+}\left(\mathbb{H}^{n+1}\right)$. The group $\Gamma$ is said to be convex co-compact if it has a finite-sided convex polyhedron $\mathcal{F}$ as a fundamental domain with the property that $\overline{\mathcal{F}} \cap \Lambda_{\Gamma}=\emptyset$. In view of the previous section, this is equivalent to requiring that $\mathcal{F}$ has no cusp point. A direct consequence of this definition is that $\Gamma$ has no parabolic elements. Moreover, in order for the quotient $\Gamma \backslash \mathbb{H}^{n+1}$ to be a Riemannian manifold, we must add the condition that $\Gamma$ has no elliptic element (i.e., $\Gamma$ must be torsion free). We call the resulting manifold $X=\Gamma \backslash \mathbb{H}^{n+1}$ a convex co-compact hyperbolic manifold.

Note that the name "convex co-compact" comes from the fact that in this case the convex core of $X$ is compact. Indeed, a direct consequence of the definition above is the absence of cusps in $X$, which, by Theorem 2.9 , implies that $C(X)$ is compact. 


\section{Classical Schottky groups}

Let $B_{1}, B_{1}^{\prime}, B_{2}, B_{2}^{\prime}, \ldots, B_{k}, B_{k}^{\prime}$ be $2 k$ Euclidean balls in $\mathbb{B}^{n+1}$ with pairwise disjoint closures and such that their boundary spheres intersect $\partial \mathbb{B}^{n+1}$ perpendicularly. Moreover, let $T_{j}$ be the hyperbolic isometry mapping $\mathbb{B}^{n+1}-\overline{B_{j}}$ onto $\overline{B_{j}^{\prime}}$. The set $\left\{T_{1}, \ldots, T_{k}\right\}$ generates a free discrete subgroup of hyperbolic isometries. (See, e.g., Theorem 12.2.17 in [34].) Furthermore, $\Gamma$ is convex co-compact. (This follows, for example, from Theorem 12.2.18 and Theorem 12.2.19 in [34], along with the results of the previous subsections.) Any discrete subgroup of $\operatorname{Isom}_{+}\left(\mathbb{H}^{n+1}\right)$ that is constructed this way is said to be a classical Schottky group.

The manifolds generated by the classical Schottky groups represent a large class of examples of convex co-compact hyperbolic manifolds. (See Remark 2.5 below.) In dimension 2, they even exhaust all examples of such manifolds.

Theorem 2.10 (Button [9]). If $X$ is a convex co-compact hyperbolic surface, then there exists a classical Schottky group $\Gamma$ such that $X \cong \Gamma \backslash \mathbb{H}^{2}$.

Proof. The theorem proved by Button in [9] is not stated in terms of hyperbolic surfaces. For a proof of the version of the theorem stated here, see, e.g., Theorem 15.2 in [7].

Remark 2.1. Theorem 2.10 does not hold in higher dimensions. Indeed, it was shown by Maskit [27] that a finitely generated Kleinian group is Schottky if and only if it is a free group of hyperbolic elements. However, it is known that there exist examples of geometrically finite (hence finitely generated, see, e.g., Theorem 12.4 .9 in [34]) Kleinian groups of hyperbolic elements which are not free.

\section{Conformally compact manifolds}

When $\Gamma$ is a group of the second kind, the convex co-compact hyperbolic manifold $\Gamma \backslash \mathbb{H}^{n+1}$ is of infinite volume, and thence not compact. However, it can be compactified in the following way. If $\mathcal{F}$ is a fundamental domain for $\Gamma$, then its Euclidean closure in $\mathbb{B}^{n+1}$ gives rise to a compact manifold with boundary when quotiented by $\Gamma$. Since $\overline{\mathcal{F}} \cap \Lambda_{\Gamma}=\emptyset$, the 
compactification of a convex co-compact hyperbolic manifold is given by

$$
\bar{X}:=\Gamma \backslash\left(\mathbb{H}^{n+1} \cup \Omega_{\Gamma}\right) .
$$

with boundary $\partial \bar{X}$, which can be identified with $\Gamma \backslash \Omega_{\Gamma}$. Moreover, it can be shown that the hyperbolic metric of a convex co-compact manifold is conformal to a metric which extends to a smooth metric on $\bar{X}$. To be more precise, there exists a non-negative function $x \in C^{\infty}(\bar{X})$ vanishing precisely on $\partial \bar{X}$ with the property that $h=x^{2} g$ is a $C^{\infty}$ Riemannian metric on $\bar{X}$ and $|d x|_{h}=1$ on $\partial \bar{X}$. (For the construction of such a function $x$ in dimension 2, see $\S 6.1$ in [7].) The function $x$ is called a smooth boundary defining function and the boundary $\partial \bar{X}$ will be referred to as the conformal boundary. In general, any manifold which can be compactified as above through the use of a boundary defining function is said to be conformally compact.

\subsection{Spectral theory of infinite-volume hyperbolic manifolds}

In this section, we review important facts from the spectral theory of the Laplacian on hyperbolic manifolds. For generalities about the Laplace operator on Riemannian manifolds, the reader is urged to consult introductory texts on this topic, such as [11] or [5]. We begin, in $\S 2.2 .1$, with a short account of the spectral theory of finite volume hyperbolic manifolds. The facts presented there are not required in order to understand any other part of this work. However, they should help to put into context the problem discussed in this thesis. Starting from the second subsection, we specialize to the case of interest, namely infinitevolume hyperbolic manifolds. The reader already familiar with this theory should at least have a look at $\S 2.2 .4$, in which the Eisenstein series, our main object of study, are introduced. Some important lemmas which will be used throughout the rest of the text will be presented there.

\subsubsection{Finite volume}

When the quotient $X=\Gamma \backslash \mathbb{H}$ is of finite volume, the spectrum of the Laplacian can take two essentially distinct forms, depending on whether $X$ is compact or not. We will then consider the two different cases separately. 


\section{Compact}

When $X$ is compact, the Laplacian has a pure discrete spectrum.

Theorem 2.11. Let $X$ be a compact Riemannian manifold. Then the spectrum of the Laplacian consists of a discrete sequence of eigenvalues

$$
0=\lambda_{0}<\lambda_{1} \leq \lambda_{2} \leq \cdots<\infty
$$

Moreover, each eigenvalue $\lambda_{i}$ has finite multiplicity and the sequence of eigenfunctions $\left\{\varphi_{0}\right\}_{i=1}^{\infty}$ corresponding to $\left\{\lambda_{i}\right\}_{i=0}^{\infty}$ forms an orthogonal basis for $L^{2}(X)$.

The proof of this result is a classic argument involving the spectral theorem for compact self-adjoint operators. It can be found in many introductory texts about the Laplacian on Riemannian manifolds. See, e.g., [5]. For an alternative derivation of this result making use of the heat kernel, see [11, Chapter VI] or [8, Chapter 7].

In the case where the geodesic flow on $X$ is ergodic, i.e., when all subsets of the unit tangent bundle of $X$ which are invariant under the geodesic flow are either of measure zero or one (with respect to the Liouville measure), the eigenfunctions are known to be equidistributed.

Theorem 2.12 (Schnirelman [38], Colin de Verdière [12], Zelditch [45]). If the geodesic flow on $X$ is ergodic, then for any orthonormal sequence of eigenfunctions $\left\{\varphi_{i}\right\}_{i=1}^{\infty}$ there exists a subsequence $\left\{\varphi_{i_{j}}\right\}_{j=1}^{\infty}$ of density 1 , i.e.,

$$
\frac{\#\left\{i_{j}: j \leq N\right\}}{N} \longrightarrow 1, \quad \text { as } j \longrightarrow \infty
$$

such that for all $a \in L^{2}(M)$,

$$
\lim _{j \rightarrow \infty} \int_{X} a(m)\left|\varphi_{j}(m)\right|^{2} d v_{X}(m)=\int_{X} a(m) d v_{X}(m),
$$

where $d v_{X}$ is the normalized volume measure on $X$.

Remark 2.2. Since the work of Hopf [19], it is a well-known fact that the geodesic flow is ergodic on compact manifolds of negative curvature. In particular, the geodesic flow is 
ergodic on compact hyperbolic manifolds. The equidistribution of Eisenstein series proved by Guillarmou and Naud and stated in $§ 1.1$ (see (1.1)) can then be seen as an analogue of the theorem above in the setting of convex co-compact hyperbolic manifolds.

Remark 2.3. The theorem of Schnirelman [38], Colin de Verdière [12] and Zelditch [45] was originally proved in greater generality and actually holds for pseudodifferential operators.

\section{Non-compact}

When $X$ is a non-compact manifold, the situation appears to be much more complicated. For this reason, in what follows, we will restrict ourselves to the hyperbolic case. In other words, $X$ is now a hyperbolic quotient $\Gamma \backslash \mathbb{H}^{n+1}$ of finite volume with finitely-many cusps. In this setting, the spectrum of the Laplacian is not strictly discrete anymore. We have the following spectral theorem.

Theorem 2.13. If $X$ is a non-compact finite volume hyperbolic quotient, then the Laplacian has an absolutely continuous spectrum $\left[n^{2} / 4, \infty\right)$ with a possible set of embedded eigenvalues and a discrete spectrum which consists of finitely many eigenvalues in the interval $\left[0, n^{2} / 2\right)$.

This result goes back to the classic work of Selberg [37]. A proof of this theorem can generally be found in introductory texts on this topic. See, for example, [20] (on surfaces only). The reader who would be interested in a different proof which avoids the use of Eisenstein series can consult [24].

The question about the existence and especially the quantity of embedded eigenvalues has not been settled yet. It was shown that there exists hyperbolic surfaces (for example, as shown by A. Selberg, the modular surface $\left.\operatorname{PSL}(2, \mathbb{Z}) \backslash \mathbb{H}^{2}\right)$ with infinitely many embedded eigenvalues. Moreover, it was conjectured by Roelcke and Selberg that for any Fuchsian subgroup $\Gamma \leq \operatorname{PSL}(2, \mathbb{R})$ of the first kind, the continuous spectrum of the Laplacian on the surface $\Gamma \backslash \mathbb{H}^{2}$ has infinitely many eigenvalues. (See, e.g., Chapter 8 in [41] for more information about this conjecture.) On the other hand, Phillips and Sarnak [13, 32] put forward the conjecture that the generic co-finite subroup $\Gamma \leq \operatorname{PSL}(2, \mathbb{R})$ gives rise to a surface on which the Laplacian has only finitely many embedded eigenvalues. 
The continuous spectrum $\left[0, n^{2} / 2\right)$ is represented by some non- $L^{2}$ functions defined as follows. As was seen in the previous section, a co-finite hyperbolic quotient can have finitely many cusps, which are associated with points on the boundary $\mathbb{R}^{n} \cup\{\infty\}$ of the hyperbolic space. Let $c_{1}, c_{2}, \ldots, c_{k}$ be a full set of inequivalent such cusp points. Moreover, for each cusp, let $\Gamma_{j}$ be the stabilizer of the cusp point $c_{j}$. The Eisenstein series on $X$ associated with the cusp $c_{j}$ is defined by

$$
E_{X}(s ; m, j)=\sum_{\gamma \in \Gamma_{j} \backslash \Gamma}\left(\operatorname{Im}\left(\sigma_{j}^{-1} \gamma m\right)\right)^{s}, \quad m \in \mathbb{H}^{n+1}, s \in \mathbb{C}, \operatorname{Re}(s)>n,
$$

where $\sigma_{j}$ is the orientation-preserving isometry of the hyperbolic space which takes the point at infinity to the cusp point $c_{j}$, i.e., $\sigma_{j} \in \operatorname{Isom}_{+}\left(\mathbb{H}^{n+1}\right)$ and $\sigma_{j}(\infty)=c_{j}$. The Eisenstein series $E_{X}(s ; m, j)$ can be extended meromorphically in the variable $s$ to the whole complex plane. Moreover, this function is analytic on the line $\operatorname{Re}(x)=\frac{n}{2}$. (See, e.g., Chapter 6 of [20] for a proof of these facts when $X=\Gamma \backslash \mathbb{H}^{2}$.) One can check that with the definition above, $E_{X}(s ; m, j)$ are smooth and satisfies the usual eigenvalue equation

$$
\Delta_{X} E_{X}(s ; m, j)=s(n-s) E_{X}(s ; m, j) .
$$

With $s:=\frac{n}{2}+i \lambda$ and $\lambda \in \mathbb{R}$, the coefficient $s(n-s)$ on the right-hand side of the last equation becomes $\frac{n^{2}}{4}+\lambda^{2} \in\left(\frac{n^{2}}{4}, \infty\right)$. Hence the functions $E_{X}(n / 2+i \lambda ; m, j)$ parametrize the absolutely continuous part of the spectrum.

When $X$ is not compact, the picture concerning the equidistribution of eigenfunctions is not as clear as the one provided by Theorem 2.12 in the compact case. An analogue of this theorem (and, at the same time, of (1.1)) was shown by Luo and Sarnak [25] on the modular surface $X=\operatorname{PSL}(2, \mathbb{Z}) \backslash \mathbb{H}^{2}$. More precisely, they proved that for any $a \in C_{0}^{\infty}(X)$,

$$
\int_{X} a(m)\left|E_{X}(n / 2+i \lambda ; m)\right|^{2} d v_{X} \sim \frac{48}{\pi} \log (\lambda) \int_{X} a(m) d v_{X}(m),
$$

as $\lambda \rightarrow \infty$, where $E_{X}(n / 2+i \lambda ; m)$ is the Eisenstein series associated with the unique cusp of $X$. For other facts about the behaviour at high-energy of the Laplace eigenfunctions in this 
setting and other related results, the reader is encouraged to consult $\S 1.1$ and the references therein.

\subsubsection{Spectral theorem}

We now turn to the case of main interest for us, i.e., when $X$ is a hyperbolic manifold of infinite volume obtained as a quotient of $\mathbb{H}^{n+1}$ by a discrete group of hyperbolic isometries. Moreover, we will assume from now on that $\Gamma$ is geometrically finite.

As with the co-finite case, the spectrum of the Laplacian has been completely described. Theorem 2.14 (Lax and Phillips $[22,23]$ ). If $\Gamma$ is a geometrically finite, discrete subgroup of $\operatorname{Isom}_{+}\left(\mathbb{H}^{n+1}\right)$, then the Laplacian on $X=\Gamma \backslash \mathbb{H}^{n+1}$ has an absolutely continuous spectrum $\left[n^{2} / 4, \infty\right)$ of infinite uniform multiplicity and a discrete spectrum consisting of finitely many eigenvalues in the interval $\left(0, n^{2} / 4\right)$. Moreover, the Laplacian has no eigenvalue in the interval $\left[n^{2} / 4, \infty\right)$.

Remark 2.4. The absence of embedded eigenvalues in the co-infinite case is in sharp contrast with the co-finite case, in which the Laplacian can have infinitely many eigenvalues embedded in the continuous spectrum.

\subsubsection{Poincaré series}

Let us now introduce a function which will play a fundamental role in the proofs of the new results described in this thesis. If $\Gamma$ is a discrete group of hyperbolic isometries, then the Poincaré series of $\Gamma$ is the series

$$
\sum_{\gamma \in \Gamma} e^{-s d\left(m, \gamma m^{\prime}\right)}
$$

for $m, m^{\prime} \in \mathbb{H}^{n+1}$ and $s \in \mathbb{C}$. It is clear from the definition above that this series does not converge for all values of $s \in \mathbb{C}$. Fix $m, m^{\prime} \in \mathbb{H}^{n+1}$ and define

$$
s_{\Gamma}:=\inf \left\{s \geq 0: \sum_{\gamma \in \Gamma} e^{-s d\left(m, \gamma m^{\prime}\right)}<\infty\right\} .
$$

The elements of $\Gamma$ being isometries, a simple application of the triangle inequality to $d\left(m, \gamma m^{\prime}\right)$ and $d\left(\gamma m, m^{\prime}\right)$ is sufficient to draw the conclusion that $s_{\Gamma}$ does not depend on the points 
$m, m^{\prime} \in \mathbb{H}^{n+1}$. The resulting non-negative number $s_{\Gamma}$ is called the exponent of convergence of $\Gamma$.

It is well-known since Poincaré [33] that $s_{\Gamma} \leq n$. Later, the work of Beardon [2, 3] (in the case where $n=1$ ) has brought to our knowledge interesting facts about the value of $s_{\Gamma}$ for groups $\Gamma$ of the second kind. Namely, in this case, we have $0<s_{\Gamma}<n$, and $s_{\Gamma}>\frac{n}{2}$ if $\Gamma$ has parabolic elements. Moreover, in this work, Beardon drew a connection between the the exponent of convergence of a Fuchsian group $s_{\Gamma}$ and the Hausdorff dimension of its limit set $\delta_{\Gamma}$. More precisely, he showed that $s_{\Gamma} \geq \delta_{\Gamma}$. This relationship between $s_{\Gamma}$ and $\delta_{\Gamma}$ has later been strengthened by Patterson and Sullivan. The part of their discoveries which will be of importance for us is summarized in the following theorem.

Theorem 2.15 (Patterson [30, 31] and Sullivan [40]). Let $\Gamma$ be a discrete subgroup of hyperbolic isometries. If $\Gamma$ is geometrically finite, then

$$
s_{\Gamma}=\delta_{\Gamma}
$$

Moreover, if $\delta_{\Gamma}>\frac{n}{2}$, then $\delta_{\Gamma}\left(1-\delta_{\Gamma}\right)$ is the lowest eigenvalue of the Laplacian $\Delta_{X}$ on $X=\Gamma \backslash \mathbb{H}^{n+1}$. On the other hand, if $\delta_{\Gamma} \leq \frac{1}{2}$, then the discrete spectrum of $\Delta_{X}$ is empty.

Remark 2.5. In [2], Beardon also showed that for any $\epsilon>0$, one can construct a Schottky group generated by two elements such that $0<s_{\Gamma}<\epsilon$. In view of the previous theorem, this provides us with examples of non-elementary manifolds satisfying the assumptions of the results presented in $\S 1.2$.

We conclude this discussion about the Poincaré series with the statement of a theorem providing a bound for the lattice-point problem in hyperbolic space. This result will reveal useful when proving Theorem 1.3.

Theorem 2.16 (Patterson [31]). For $T \geq 0$, let

$$
N(T):=\#\{\gamma \in \Gamma: d(0, \gamma 0) \leq T\}
$$


If $\Gamma$ is convex co-compact, then

$$
N(T)=\mathcal{O}\left(e^{\delta_{\Gamma} T}\right)
$$

\subsubsection{Eisenstein series}

Let $X$ be a geometrically finite hyperbolic manifold. The resolvent of the Laplacian $\Delta_{X}$ on $X$, is defined by

$$
R_{X}(s):=\left(\Delta_{X}-s(n-s)\right)^{-1}
$$

as a bounded operator on $L^{2}(X)$ for $\operatorname{Re}(s)>\frac{n}{2}$, when $s(n-s)$ is not an eigenvalue of $\Delta_{X}$. This family of operators extends to the complex plane in the following way.

Theorem 2.17 (Guillarmou and Mazzeo [15]). If $X$ is a geometrically finite hyperbolic manifold, then the family of operators $R_{X}(s)$ defined above extends meromorphically to a family of continuous operators

$$
R_{X}(s): C_{0}^{\infty}(X) \longrightarrow C^{\infty}(X), \quad s \in \mathbb{C},
$$

with poles of finite rank.

Remark 2.6. This theorem has been first proved for convex co-compact hyperbolic manifolds by Mazzeo and Melrose [29]. Their statement was originally in terms of asymptotically hyperbolic manifolds, but appeared later to be erroneous [14].

From now on, we will make the additional assumption that $X$ is convex co-compact. Using the same normalization factor as in [16], the Eisenstein series on $X$ associated with $\xi \in \partial \bar{X}$ is defined for the boundary defining function $x$ by

$$
E(s ; m, \xi):=\frac{2 \pi^{\frac{n}{2}} \Gamma\left(s-\frac{n}{2}+1\right)}{2^{-s} \Gamma(s)} \lim _{m^{\prime} \rightarrow \xi} x\left(m^{\prime}\right)^{-s} R_{X}\left(s ; m, m^{\prime}\right), \quad s \in \mathbb{C},
$$

where $R_{X}\left(s ; m, m^{\prime}\right)$ is the resolvent integral kernel and $\Gamma$ is the usual gamma function. By [17, 29], for any $m \in X$, we have $x^{-s}(\cdot) R_{X}(s, m, \cdot) \in C^{\infty}(\bar{X})$ and similarly $x^{-s}(\cdot) R_{X}(s, \cdot, m) \in$ 
$C^{\infty}(\bar{X})$. It follows that $E(s ; \cdot, \cdot) \in C^{\infty}(X \times \partial \bar{X})$. Notice that the definition of the Eisenstein series depends on the choice of the boundary defining function; see also Remark 2.8 below.

Here, we dwell briefly on the case where $X=\mathbb{H}^{n+1}$ since it will be of particular importance in what follows. The reader is referred to $[16, \S 2.2]$ and $[17, \S 2]$ for more details. The resolvent kernel is given by

$$
\begin{aligned}
R_{\mathbb{H}^{n+1}}\left(s ; m, m^{\prime}\right) & =\frac{\pi^{-\frac{n}{2}} 2^{-2 s-1} \Gamma(s)}{\Gamma\left(s-\frac{n}{2}+1\right)} \cosh ^{-2 s}\left(\frac{d\left(m, m^{\prime}\right)}{2}\right) \\
& \times F\left(s, s-\frac{n-1}{2}, 2 s-n+1 ; \cosh ^{-2}\left(\frac{d\left(m, m^{\prime}\right)}{2}\right)\right),
\end{aligned}
$$

where $F$ is the usual hypergeometric function, i.e.,

$$
F(a, b, c ; z):=\frac{\Gamma(c)}{\Gamma(b) \Gamma(c-b)} \int_{0}^{1} t^{b-1}(1-t)^{c-b-1}(1-t z)^{-a} d t .
$$

Define

$$
x(m):=2 \frac{1-|m|}{1+|m|}, \quad m \in \mathbb{B}^{n+1} .
$$

It is easy to check from (2.1) that $x$ is a boundary defining function for $\mathbb{B}^{n+1}$. Moreover, using the expression of the hyperbolic distance (2.2), one can see that

$$
x(m)=2 e^{-d(m, 0)} .
$$

Combining $(2.2),(2.12),(2.13)$ and (2.15), we obtain the following expression for the Eisenstein series on $\mathbb{H}^{n+1}$ associated with $\xi \in S^{n}$ :

$$
E_{0}(s ; m, \xi):=\left(\frac{1-|m|^{2}}{|m-\xi|^{2}}\right)^{s}=e^{s \phi_{\xi}(m)}, \quad s \in \mathbb{C} .
$$

where $\phi_{\xi}(m):=\log \left(\frac{1-|m|^{2}}{|m-\xi|^{2}}\right)$ will be called the Busemann function.

We now state a lemma proved by Guillarmou and Naud, which describes the action of isometries on the Eisenstein series. Since the argument is simple and we will make extensive use of this result later, we reproduce the proof below. 
Lemma 2.18 (Guillarmou and Naud [16]). Let $\gamma \in \operatorname{Isom}_{+}\left(\mathbb{H}^{n+1}\right)$, then for all $m \in \mathbb{B}^{n+1}$ and $\xi \in S^{n}$,

$$
E_{0}(s ; \gamma m, \gamma \xi)=E_{0}(s ; m, \xi)|D \gamma(\xi)|^{-s}
$$

where $D_{m} \gamma(\xi)$ is the limit as $m^{\prime} \rightarrow \infty$ of the differential of $\gamma$ at the point $m^{\prime}$.

Proof. We follow the argument of the proof of Lemma 4 in [16]. Let $\xi \in S^{n}$ and $m \in \mathbb{B}^{n+1}$. Since $\gamma$ is an isometry, for all $m^{\prime} \in \mathbb{B}^{n+1}$, we have $d\left(\gamma m, \gamma m^{\prime}\right)=d\left(m, m^{\prime}\right)$. It follows from (2.2) that

$$
\frac{\left|\gamma m-\gamma m^{\prime}\right|}{\left(1-|\gamma m|^{2}\right)\left(1-\left|\gamma m^{\prime}\right|^{2}\right)}=\frac{\left|m-m^{\prime}\right|}{\left(1-|m|^{2}\right)\left(1-\left|m^{\prime}\right|^{2}\right)}
$$

for all $m^{\prime} \in \mathbb{B}^{n+1}$. This equation can be rearranged as

$$
\left(\frac{1-|\gamma m|^{2}}{\left|\gamma m-\gamma m^{\prime}\right|^{2}}\right) /\left(\frac{1-|m|^{2}}{\left|m-m^{\prime}\right|^{2}}\right)=\frac{1-\left|m^{\prime}\right|^{2}}{1-\left|\gamma m^{\prime}\right|^{2}}
$$

Hence,

$$
\frac{E_{0}(s ; \gamma m, \gamma \xi)}{E_{0}(s ; m, \xi)}=\frac{\lim _{m^{\prime} \rightarrow \xi}\left(\frac{1-|\gamma m|^{2}}{\left|\gamma m-\gamma m^{\prime}\right|^{2}}\right)^{s}}{\lim _{m^{\prime} \rightarrow \xi}\left(\frac{1-|m|^{2}}{\left|m-m^{\prime}\right|^{2}}\right)^{s}}=\lim _{m^{\prime} \rightarrow \xi}\left(\frac{1-\left|m^{\prime}\right|^{2}}{1-\left|\gamma m^{\prime}\right|^{2}}\right)^{-s}=|D \gamma(\xi)|^{-s}
$$

which is the statement of the lemma.

It is easily seen (e.g., passing to the half-space model and using the previous lemma) that the Busemann function $\phi_{\xi}(m)$ is constant on the horospheres tangent to $\partial \mathbb{H}^{n+1}$ at $\xi$. Moreover, the hyperbolic gradient of $\phi_{\xi}$, denoted by $\tilde{\nabla}_{m} \phi_{\xi}(m)$, is of Euclidean norm 1 for all $m \in \mathbb{H}^{n+1}$, and it points in the direction of the unique geodesic joining $m$ to $\xi$.

The following lemma gives an upper bound on the norm of the derivatives of the Eisenstein series on $\mathbb{H}^{n+1}$ when acted on by $\Gamma$. The derivatives appearing in the statement of the lemma are with respect to the variable $m$. (See (2.7) for an explanation of the notation used.) 
Lemma 2.19. For $\xi \in \Omega_{\Gamma}, \gamma \in \Gamma$ and $m$ in a compact subset $K$ of $\mathbb{B}^{n+1}$,

$$
\left|\partial_{\alpha} E_{0}(1 ; \gamma m, \xi)\right| \leq C e^{-d(0, \gamma 0)}, \quad \alpha \in \mathbb{N}_{0}^{n+1},
$$

for some $C>0$ depending only on $K, \xi$ and $\alpha$.

Proof. By Lemma 2.18, we have

$$
\left|D \gamma\left(\gamma^{-1} \xi\right)\right|^{-1}=E_{0}(1 ; \gamma m, \xi)\left(E_{0}\left(1 ; m, \gamma^{-1} \xi\right)\right)^{-1}=\frac{1-|\gamma m|^{2}}{|\gamma m-\xi|^{2}} \frac{\left|m-\gamma^{-1} \xi\right|^{2}}{1-|m|^{2}}
$$

Since $\xi$ is not in the limit set of $\Gamma$, we can find $\epsilon>0$ uniform in $\gamma$, such that $|\gamma m-\xi|^{2}>$ $\epsilon$. Therefore, for any $m \in K$, we have $\left|D \gamma\left(\gamma^{-1} \xi\right)\right|^{-1} \leq C_{1}\left(1-|\gamma m|^{2}\right)$ for some $C_{1}>0$ independent of $\gamma$. On the other hand, by Lemma 2.4, we find that $1-|\gamma m|^{2} \leq C_{2} e^{-d(0, \gamma 0)}$ for some $C_{2}>0$ independent of $\gamma$. Hence

$$
\left|D \gamma\left(\gamma^{-1} \xi\right)\right|^{-1} \leq C e^{-d(0, \gamma 0)}
$$

for some constant $C$ independent of $\gamma$. Since $\partial_{\alpha} E_{0}\left(1 ; m, \gamma^{-1} \xi\right)$ is bounded uniformly with respect to $\gamma$ for $m \in K$, it follows from the last inequality that

$$
\left|\partial_{\alpha} E_{0}(1 ; \gamma m, \xi)\right|=\left.\left|\left(\partial_{\alpha} E_{0}\left(1 ; m, \gamma^{-1} \xi\right)\right)\right| D \gamma\left(\gamma^{-1} \xi\right)\right|^{-1} \mid \leq C_{\alpha} e^{-d(0, \gamma 0)}
$$

where $C_{\alpha}>0$ depends only on $K, \xi$ and $\alpha$.

Remark 2.7. Notice that when $\xi \in \overline{\mathcal{F}} \cap S^{n}$, since $\Gamma$ is convex co-compact the Euclidean distance between $\gamma m$ and $\xi$ can be bounded from below uniformly in $\xi$. Therefore, in this case, the constant $C$ appearing in the statement of Lemma 2.19 is independent of $\xi$.

The terminology Eisenstein "series" stems from the fact that the function defined by (2.12) proves to lift, under precise circumstances, to an absolutely convergent series on $\mathbb{H}^{n+1}$. This is attested by the following lemma, which was proved by Guillarmou and Naud. Since the implications of this result are fundamental to our purposes, we recall its proof below.

Lemma 2.20 (Guillarmou and Naud [16]). Let $X:=\Gamma \backslash \mathbb{H}^{n+1}$ be a convex co-compact hyperbolic manifold with $\delta_{\Gamma}<n / 2$ and let $\pi_{\Gamma}:=\mathbb{B}^{n+1} \rightarrow \Gamma \backslash \mathbb{B}^{n+1}$ be the quotient projection map. 
If $\xi \in \Omega_{\Gamma}$ and $m \in \mathbb{H}^{n+1}$, then for any complex number $s$ such that $\operatorname{Re}(s)>\delta_{\Gamma}$, the series

$$
\sum_{\gamma \in \Gamma} E_{0}(s ; \gamma m, \xi)
$$

converges absolutely and is invariant under the action of $\Gamma$ as a function of $m \in \mathbb{H}^{n+1}$. Moreover, we have

$$
E\left(s ; \pi_{\Gamma}(m), \pi_{\Gamma}(\xi)\right)=\sum_{\gamma \in \Gamma} E_{0}(s ; \gamma m, \xi)
$$

where $E(s ; \cdot, \cdot)$ is defined as in (2.12) with a boundary defining function $x$ so that, in a neighbourhood of $\xi$, the non-negative function $x\left(\pi_{\Gamma}(\cdot)\right)$ on $\overline{\mathbb{B}^{n+1}}$ is given by (2.14).

Proof. We follow the argument of the proof of Lemma 5 in [16]. Since $\xi \notin \Lambda_{\Gamma}$, there exists $\epsilon_{\xi, m}>0$ independent of $\gamma$, such that $|\gamma m-\xi|>\epsilon_{\xi, m}$ for all $\gamma \in \Gamma$. It follows from (2.14), (2.15) and (2.16) that

$$
\left|E_{0}(s ; \gamma m, \xi)\right| \leq C_{s, m, \xi} e^{-s d(\gamma m, 0)}
$$

for some positive constant $C_{s, m, \xi}$ independent of $\gamma$. By (2.11) and Theorem 2.15, the series (2.17) converges whenever $\operatorname{Re}(s)>\delta_{\Gamma}$.

For $\operatorname{Re}(s)>\frac{n}{2}$, the lift to $\mathbb{H}^{n+1} \times \mathbb{H}^{n+1}$ of the resolvent kernel on $\Gamma \backslash \mathbb{H}^{n+1}$ is given by (see, e.g., $[29])$

$$
R_{X}\left(s ; \pi_{\Gamma}(m), \pi_{\Gamma}\left(m^{\prime}\right)\right)=\sum_{\gamma \in \Gamma} R_{\mathbb{H}^{n+1}}(s ; \gamma m, \xi)
$$

whenever $\pi_{\Gamma}(m) \neq \pi_{\Gamma}\left(m^{\prime}\right)$. It follows again from (2.11) and Theorem 2.15, in conjunction with the expression of $R_{\mathbb{H}^{n+1}}$ given by (2.13), that this series converges absolutely for $\operatorname{Re}(s)>$ $\delta_{\Gamma}$. Moreover, it converges uniformly on compact subsets of the product $\mathbb{H}^{n+1} \times \mathbb{H}^{n+1} \backslash$ diag. 
Since $\lim _{m \rightarrow \xi} x\left(\pi_{\Gamma}(m)\right)=\lim _{m \rightarrow \xi} 2 \frac{1-|m|}{1+|m|}$, we have by (2.12), (2.14), (2.16) and (2.18), that

$$
\begin{aligned}
E\left(s ; \pi_{\Gamma}(m), \pi_{\Gamma}(\xi)\right) & =\frac{2 \pi^{\frac{n}{2}} \Gamma\left(s-\frac{n}{2}+1\right)}{2^{-s} \Gamma(s)} \sum_{\gamma \in \Gamma} \lim _{m^{\prime} \rightarrow \xi}\left(R_{\mathbb{H}^{n+1}}\left(s ; \gamma m, m^{\prime}\right)\left(2 \frac{1-\left|m^{\prime}\right|}{1+\left|m^{\prime}\right|}\right)^{-s}\right) \\
& =\sum_{\gamma \in \Gamma} E_{0}(s ; \gamma m, \xi),
\end{aligned}
$$

which concludes the proof.

Remark 2.8. If $\pi_{\gamma}^{*}(x)$ is not given by (2.14), one can see from (2.12) that the Eisenstein series $E(s ; m, \xi)$ can be multiplied by the function $\eta^{-s}(\xi)$, where $\eta(\xi):=2 \lim _{m \rightarrow \xi} \frac{1-|m|}{(1+|m|) \tilde{x}(m)}$, in order for it to admit a lift to $\mathbb{H}^{n+1}$ as an absolutely convergent series as in (2.17). 


\section{CHAPTER 3 \\ The moments at high-energy}

This chapter is devoted to the determination of the moments of Eisenstein series at highenergy. As explained in the introduction, this is essentially the content of Theorem 1.1, which will be proved in $\S 3.1$. When $p$ is even, the expression of the limit as $\lambda \rightarrow \infty$ of $I_{p / 2}^{p}(\lambda, \xi)$ that we obtain depends on the partition lattice of a $p / 2$-element set. In $\S 3.2$, we explain how to calculate explicitly the coefficients $C_{\theta}$ 's appearing in $f_{p}(m, \xi)$ when $p$ is even, and we consider in detail the examples $p=6$ and $p=8$. In the last section, we derive from Theorem 1.1 the high-energy limit of the moments of the real and imaginary parts of Eisenstein series.

\subsection{Proof of Theorem 1.1}

We lift everything to the universal cover of $X$ and we work in a fixed fundamental domain for $\Gamma$. To be more precise, let $\mathcal{F}$ be a fundamental domain for $\Gamma$ in $\mathbb{H}^{n+1}$. When no confusion is likely to arise, the restriction to $\mathcal{F}$ of the lift to $\mathbb{H}^{n+1}$ of the function $a$ will also be denoted by $a$. Since we will be interested in the value of the integral over $\mathcal{F}$, we can extend this function to $\mathbb{H}^{n+1}$ by defining it to be zero everywhere outside of $\mathcal{F}$. Note that with this notation, supp $a$ is compact in $\mathbb{H}^{n+1}$. The point $\xi$ in the conformal boundary of $X$ lifts to a point, which will also be denoted by $\xi$, in $\Omega_{\Gamma} \cap \overline{\mathcal{F}}$. Let $x$ be the boundary-defining function of $\bar{X}$ used to define $E\left(s_{\lambda} ; m, \xi\right)$ and let $\tilde{x}$ be its lift to $\mathbb{H}^{n+1}$. Let $\pi_{\Gamma}:=\mathbb{H}^{n+1} \rightarrow \Gamma \backslash \mathbb{H}^{n+1}$

be the quotient projection map and define $\eta(\xi):=2 \lim _{m \rightarrow \xi} \frac{1-|m|}{(1+|m|) \tilde{x}(m)}$, as in Remark 2.8, so that we can apply Lemma 2.20 to write $E\left(s ; \pi_{\Gamma}(m), \pi_{\Gamma}(\xi)\right)$ in the form of a series:

$$
E\left(s_{\lambda} ; \pi_{\Gamma}(m), \pi_{\Gamma}(\xi)\right)=\sum_{\gamma \in \Gamma} E_{0}\left(s_{\lambda} ; \gamma m, \xi\right) \eta(\xi)^{s_{\lambda}}, \quad m \in \mathcal{F}
$$


We then have

$$
\begin{aligned}
E^{k} \bar{E}^{p-k} & =\eta(\xi)^{\frac{p n}{2}+i(2 k-p) \lambda} \prod_{l=1}^{k}\left(\sum_{\gamma_{l} \in \Gamma} E_{0}\left(s_{\lambda} ; \gamma_{l} m, \xi\right)\right) \prod_{l=p-k+1}^{p}\left(\sum_{\gamma_{l} \in \Gamma} E_{0}\left(\bar{s}_{\lambda} ; \gamma_{l} m, \xi\right)\right) \\
& =\eta(\xi)^{\frac{p n}{2}+i(2 k-p) \lambda} \sum_{\gamma \in S}\left(\prod_{l=1}^{p} E_{0}\left(\frac{n}{2} ; \gamma_{l} m, \xi\right)\right) e^{i \lambda \psi_{\gamma}(m)},
\end{aligned}
$$

where $\gamma=\left(\gamma_{1}, \ldots, \gamma_{p}\right) \in S:=\underbrace{\Gamma \times \cdots \times \Gamma}_{p \text { times }}$, and

$$
\psi_{\gamma}(m):=\log \left(\frac{E_{0}\left(1 ; \gamma_{1} m, \xi\right) \cdots E_{0}\left(1 ; \gamma_{k} m, \xi\right)}{E_{0}\left(1 ; \gamma_{k+1} m, \xi\right) \cdots E_{0}\left(1 ; \gamma_{p} m, \xi\right)}\right)
$$

Let us now decompose $\underbrace{\Gamma \times \cdots \times \Gamma}_{p \text { times }}$ into two distinct parts:

$$
S=S_{1} \sqcup S_{2},
$$

where $\gamma \in S_{1}$ if $\psi_{\gamma}(m)=0$ for all $m \in \mathbb{H}^{n+1}$, and $\gamma \in S_{2}$ otherwise. The sets $S_{1}$ and $S_{2}$ will be described more explicitly in $§ 3.1 .2$. With this notation, we can split $I_{k}^{p}(\lambda, \xi)$ into two parts:

$$
\begin{aligned}
I_{k}^{p}(\lambda, \xi) & =\int_{X} a(m) \eta(\xi)^{\frac{p n}{2}+i(2 k-p) \lambda} \sum_{\gamma \in S_{1}}\left(\prod_{l=1}^{p} E_{0}\left(\frac{n}{2} ; \gamma_{l} m, \xi\right)\right) d v(m) \\
& +\eta(\xi)^{\frac{p n}{2}+i(2 k-p) \lambda} \sum_{\gamma \in S_{2}} I_{\gamma}(\lambda) .
\end{aligned}
$$

where

$$
I_{\gamma}(\lambda):=\int_{X} a(m)\left(\prod_{l=1}^{p} E_{0}\left(\frac{n}{2} ; \gamma_{l} m, \xi\right)\right) e^{i \lambda \psi_{\gamma}(m)} d v(m)
$$

Notice that $\left|\eta(\xi)^{\frac{p n}{2}+i(2 k-p) \lambda}\right|=|\eta(\xi)|^{\frac{p n}{2}}$ does not depend on $\lambda$.

We will prove the theorem in two steps. First, we show that the sum of oscillatory integrals in (3.1) converges to 0 as $\lambda \rightarrow \infty$. Then, we show that the first integral in the expression above is equal to the right-hand side of (1.5). 


\subsubsection{The sum over $S_{2}$}

The following lemma shows that each oscillatory integral $I_{\gamma}(\lambda)$ in the series above vanishes at high-energy.

Lemma 3.1. For all $\gamma \in S_{2}$,

$$
I_{\gamma}(\lambda) \longrightarrow 0
$$

as $\lambda \rightarrow \infty$.

Proof. Let $\epsilon>0$. In order to study the behaviour of $I_{\gamma}(\lambda)$ for large values of $\lambda$, we need to know more about the set of points at which the gradient of the phase function $\psi_{\gamma}$ vanishes. By Lemma 2.18,

$$
\psi_{\gamma}(m)=\sum_{j=1}^{k} \phi_{\xi_{j}}(m)-\sum_{j=1}^{p-k} \phi_{\xi_{j+k}}(m)+\sum_{j=1}^{k} \log \left|D \gamma_{j}\left(\xi_{j}\right)\right|-\sum_{j=1}^{p-k} \log \left|D \gamma_{j+k}\left(\xi_{j+k}\right)\right|
$$

where $\xi_{j}:=\gamma_{j}^{-1} \xi \in S^{n}$ and $\phi_{\xi}(m)$ is the Busemann function defined in $\S 2.2 .4$. The only terms depending on $m$ on the right-hand side of this equation are the $\phi_{\xi}(m)$ 's. It follows that the gradient of the phase function essentially consists in the gradient of the Busemann functions. Direct calculations show that the Euclidean gradient of $\phi_{\xi}(m)$ is given by

$$
\nabla_{m} \phi_{\xi}(m)=-2\left(\frac{m}{1-|m|^{2}}+\frac{m-\xi}{|m-\xi|^{2}}\right)
$$

By (3.2) and (3.3), the gradient of the phase function $\psi_{\gamma}(m)$ satisfies

$$
\left|\nabla_{m} \psi_{\gamma}(m)\right|^{2}=\frac{F_{\gamma}\left(x_{1}, \ldots, x_{n+1}\right)}{G_{\gamma}\left(x_{1}, \ldots, x_{n+1}\right)}
$$

where $F_{\gamma}$ and $G_{\gamma}$ are polynomials in the $n+1$ variables $x_{1}, \ldots, x_{n+1}$, where $m=\left(x_{1}, \ldots, x_{n+1}\right) \in$ $\mathbb{R}^{n+1}$. Moreover, $F_{\gamma}$ is not the zero polynomial, since $\gamma \in S_{2}$. It follows that the set of stationary points $K_{\gamma}$ of $\psi_{\gamma}(m)$ is contained in the zero set of $F_{\gamma}$. Therefore, $K_{\gamma}$ is a subset of an algebraic variety of codimension at least 1 . In particular, the stationary points are all contained in a closed set of hyperbolic measure 0 . 
Let $\Omega_{\gamma}^{\delta}$ be the $\epsilon$-neighbourhood of $K_{\gamma}$ inside of supp $a$, i.e., $\Omega_{\gamma}^{\delta}:=\left(\cup_{m \in K_{\gamma}} B^{n+1}(m, \delta)\right) \cap$ supp $a$, where $B^{n+1}(m, \delta)$ is the Euclidean ball of radius $\delta$ centered at $m$. For $\delta>0$ small, the integral $I_{\gamma}(\lambda)$ can be split into two parts with the help of a smooth cut-off function $0 \leq \rho_{\gamma}^{\delta}(m) \leq 1$ which vanishes everywhere inside of $\Omega_{\gamma}^{\delta / 2}$ and is equal to 1 everywhere outside of $\Omega_{\gamma}^{\delta}$ :

$$
I_{\gamma}(\lambda)=A_{\gamma}^{\delta}(\lambda)+B_{\gamma}^{\delta}(\lambda)
$$

where

$$
A_{\gamma}^{\delta}(\lambda):=\int_{\mathbb{H}^{n+1}} a(m)\left(1-\rho_{\gamma}^{\delta}(m)\right)\left(\prod_{l=1}^{p} E_{0}\left(\frac{n}{2} ; \gamma_{l} m, \xi\right)\right) e^{i \lambda \psi_{\gamma}(m)} \frac{2^{n+1} d m}{\left(1-|m|^{2}\right)^{n+1}}
$$

and

$$
B_{\gamma}^{\delta}(\lambda):=\int_{\mathbb{H}^{n+1}} a(m) \rho_{\gamma}^{\delta}(m)\left(\prod_{l=1}^{p} E_{0}\left(\frac{n}{2} ; \gamma_{l} m, \xi\right)\right) e^{i \lambda \psi_{\gamma}(m)} \frac{2^{n+1} d m}{\left(1-|m|^{2}\right)^{n+1}}
$$

Since the integrand of $A_{\gamma}^{\delta}(\lambda)$ is bounded uniformly with respect to $\delta$ and $\lambda$, it is clear from the definition of $K_{\gamma}$ and from the remark following it that $\left|A_{\gamma}^{\delta}(\lambda)\right|$ can be made as small as we want by taking $\delta$ small enough. So, let $\delta>0$ be small enough so that $\left|A_{\gamma}^{\delta}(\lambda)\right|<\epsilon / 2$ for all $\lambda>0$.

On the other hand, applying integration by parts to the integral $B_{\gamma}^{\delta}(\lambda)$, we get

$B_{\gamma}^{\delta}(\lambda)=\frac{1}{i \lambda} \int_{\mathbb{H}^{n+1}} e^{i \lambda \psi_{\gamma}(m)} \nabla^{*}\left(a(m) \rho_{\gamma}^{\delta}(m)\left(\prod_{l=1}^{p} E_{0}\left(\frac{n}{2} ; \gamma_{l} m, \xi\right)\right) \frac{\nabla_{m} \psi_{\gamma}(m)}{\left|\nabla_{m} \psi_{\gamma}(m)\right|^{2}}\right) \frac{2^{n+1} d m}{\left(1-|m|^{2}\right)^{n+1}}$,

where $\nabla^{*}$ is the adjoint of $\nabla$ with respect to the volume measure $\frac{2^{n+1} d m}{\left(1-|m|^{2}\right)^{n+1}}$. It is then clear from the expression above that $\left|B_{\gamma}^{\delta}(\lambda)\right|$ can be made less than $\epsilon / 2$ by taking $\lambda$ large enough. Therefore,

$$
\left|I_{\gamma}(\lambda)\right| \leq\left|A_{\gamma}^{\delta}(\lambda)\right|+\left|B_{\gamma}^{\delta}(\lambda)\right|<\epsilon
$$

when $\lambda$ is large enough. 
We are now in a position to treat the sum of $I_{\gamma}(\lambda)$. Using the fact that for any $\gamma_{l} \in \Gamma$,

$$
\left|E\left(n / 2, \gamma_{l} m, \xi\right)\right| \leq C e^{-\frac{n}{2} d\left(0, \gamma_{l} 0\right)}
$$

for some constant $C>0$, when $m$ stays in a compact subset of $\mathbb{H}^{n+1}$ (see Lemma 2.19 above), it is easy to check that

$$
\sum_{\gamma \in S_{2}}\left|I_{\gamma}(\lambda)\right| \leq C^{\prime} \prod_{l=1}^{p} \sum_{\gamma_{l} \in \Gamma} e^{-\frac{n}{2} d\left(0, \gamma_{l} 0\right)}<\infty
$$

for some $C^{\prime}>0$. Notice that the last inequality follows from (2.11), Theorem 2.15 and the hypothesis that $\delta_{\Gamma}<\frac{n}{2}$. Therefore, Lemma 3.1, along with the Dominated Convergence Theorem, allows us to conclude that

$$
\sum_{\gamma \in S_{2}} I_{\gamma}(\lambda) \longrightarrow 0
$$

as $\lambda \rightarrow \infty$.

\subsubsection{The sum over $S_{1}$}

As a consequence of (3.4), the proof would be complete if we could show that the first term in (3.1) corresponds to the limit (1.5). Let us first determine a more manageable characterization of the sets $S_{1}$ and $S_{2}$.

Lemma 3.2. Let $\gamma=\left(\gamma_{1}, \ldots, \gamma_{k}, \gamma_{1}^{\prime}, \ldots, \gamma_{p-k}^{\prime}\right) \in S$. Then $\gamma \in S_{1}$ if and only if $k=\frac{p}{2}$ and there exists a permutation $\sigma$ of the set $\{1, \ldots, k\}$ such that $\gamma_{l}=\gamma_{\sigma(l)}^{\prime}$ for all $1 \leq l \leq k$.

Proof. Since the function $E_{0}(1 ; \cdot, \xi)$ is $C^{\infty}$ and never vanishes on $\mathbb{H}^{n+1}$, we have $\psi_{\gamma}(m)=0$ for all $m \in \mathbb{H}^{n+1}$ if and only if

$$
E_{0}\left(1 ; \gamma_{1} m, \xi\right) \cdots E_{0}\left(1 ; \gamma_{k} m, \xi\right)=E_{0}\left(1 ; \gamma_{1}^{\prime} m, \xi\right) \cdots E_{0}\left(1 ; \gamma_{p-k}^{\prime} m, \xi\right), \quad m \in \mathbb{H}^{n+1}
$$

This equation is clearly satisfied for all $m \in \mathbb{H}^{n+1}$ when $k=\frac{p}{2}$, if for some permutation $\sigma$ of the set $\{1, \ldots, k\}$, we have $\gamma_{l}=\gamma_{\sigma(l)}^{\prime}$ for all $1 \leq l \leq k$. 
Conversely, assuming that (3.5) holds, it follows from Lemma 2.18 and (2.16) that (3.5) can be rearranged as

$$
\prod_{l=1}^{k}\left|m-\gamma_{l}^{-1} \xi\right|=\frac{\left|D \gamma_{1}^{\prime}\left(\gamma_{1}^{\prime-1} \xi\right)\right| \cdots\left|D \gamma_{p-k}^{\prime}\left(\gamma_{p-k}^{\prime-1} \xi\right)\right|}{\left|D \gamma_{1}\left(\gamma_{1}^{-1} \xi\right)\right| \cdots\left|D \gamma_{k}\left(\gamma_{k}^{-1} \xi\right)\right|} \prod_{l=1}^{k}\left|m-\gamma_{l}^{\prime-1} \xi\right| .
$$

If for any $l \in\{1, \ldots, k\}$, we let $m \rightarrow \gamma_{l}^{-1} \xi$ in the equation above, then the left-hand side vanishes. Since this equation should be satisfied for all $m \in \mathbb{H}^{n+1}$, it follows that for at least one $j \in\{1, \ldots, p-k\}$, we have $\left|m-\gamma_{j}^{\prime-1} \xi\right| \rightarrow 0$ on the right-hand side. In other words, for some $l \in\{1, \ldots, k\}$ and some $j \in\{1, \ldots, p-k\}$, we have $\gamma_{l}^{-1} \xi=\gamma_{j}^{\prime-1} \xi$, or, equivalently, $\gamma_{j}^{\prime} \gamma_{l}^{-1} \xi=\xi$. Since the point $\xi \in \Omega_{\Gamma}$, it follows from Proposition 2.6 that $\xi$ can be fixed by the element $\gamma_{j}^{\prime} \gamma_{l}^{-1}$ of $\Gamma$ if and only if this element is the identity. Therefore, $\gamma_{j}^{\prime}=\gamma_{l}$. It follows that (3.6) can be simplified by cancelling $\left|m-\gamma_{l}^{-1} \xi\right|$ on both sides.

Applying this argument iteratively to all $\gamma_{l}^{-1} \xi$ on the left-hand side and to all $\gamma_{j}^{\prime-1} \xi$ on the right-hand side of (3.6), we conclude that $k=p-k$ and we deduce the existence of a permutation $\sigma$ of the set $\{1, \ldots, k\}$ such that $\gamma_{l}=\gamma_{\sigma(l)}^{\prime}$ for all $1 \leq l \leq k$.

An immediate consequence of Lemma 3.2 is that $S_{1}=\emptyset$ if $k \neq \frac{p}{2}$. Therefore, the limit (1.5) of Theorem 1.1 in the case where $k \neq \frac{p}{2}$ follows directly from (3.1), (3.4) and Lemma 3.2.

Now, it remains only to show (1.5) in the case where $k=\frac{p}{2}$. Therefore, for the rest of this section, we assume that $p$ is even and that $k=\frac{p}{2}$. For $\gamma \in S_{1}$, we will also make use of the notation $\gamma=\left(\gamma_{1}, \ldots, \gamma_{k}, \gamma_{1}^{\prime}, \ldots, \gamma_{k}^{\prime}\right)$ introduced in the statement of Lemma 3.2. Since for all $l$, we have $\gamma_{l}=\gamma_{j}^{\prime}$ for some $j$, it follows from (3.1) that the proof would be complete if we show that

$$
\eta(\xi)^{k n} \sum_{\gamma \in S_{1}}\left(\prod_{l=1}^{k} E_{0}\left(n ; \gamma_{l} m, \xi\right)\right)=f_{p}(m, \xi)
$$

Notice that we made used of the elementary fact that $E_{0}^{t}(s ; m, \xi)=E_{0}(t s ; m, \xi)$ in the last equation. 
Moreover, if $\gamma_{i}=\gamma_{j}$ for some $i \neq j$, then $E_{0}\left(n ; \gamma_{i} m, \xi\right) E_{0}\left(n ; \gamma_{j} m, \xi\right)=E_{0}^{2}\left(n ; \gamma_{i} m, \xi\right)$. It follows that according to the "configuration" of $\left(\gamma_{1}, \ldots, \gamma_{k}\right)$, the summands in the series appearing in (3.7) take the form

$$
\prod_{l=1}^{k} E_{0}\left(n ; \gamma_{l} m, \xi\right)=E_{0}\left(\theta_{1} n ; \gamma_{1} m, \xi\right) \cdots E_{0}\left(\theta_{r} n ; \gamma_{r} m, \xi\right)
$$

for some integer partition $\theta:=\left(\theta_{1}, \cdots, \theta_{r}\right)$ of $k$, i.e., (3.8) holds for some positive integers $\theta_{1}, \theta_{2}, \ldots, \theta_{r}$ satisfying $\theta_{1} \geq \theta_{2} \geq \cdots \geq \theta_{r}$ and

$$
k=\theta_{1}+\cdots+\theta_{r} .
$$

Let $\Theta_{l}$ be the set of all integer partitions of $k$ into exactly $l$ parts, and define $\Theta:=\bigcup_{l=1}^{k} \Theta_{l}$.

In order to prove (3.7), we will use the following approach. We will split $S_{1}$ into all the possible configuration types $\left(\theta_{1}, \ldots, \theta_{r}\right)$ of $\left(\gamma_{1}, \ldots, \gamma_{k}\right)$ and we will count "how many" of each configuration we have.

\section{Cases $k=1$ and $k=2$}

Before treating the general case, let us first illustrate the idea through some examples where $k$ is small. For $k=1$, there is only one permutation $\sigma$, that is, the identity. Therefore, the right-hand side of (3.7) becomes

$$
f_{2}(m, \xi)=\eta(\xi)^{n} \sum_{\gamma \in S_{1}} E_{0}\left(n ; \gamma_{1} m, \xi\right)=\sum_{\gamma_{1} \in \Gamma}\left(\eta(\xi)^{n} E_{0}\left(n ; \gamma_{1} m, \xi\right)\right)=E(n ; m, \xi) .
$$

For $k=2$, we have that $\gamma \in S_{1}$ if and only if $\gamma$ takes either the form $\left(\gamma_{1}, \gamma_{2}, \gamma_{1}, \gamma_{2}\right)$ or the form $\left(\gamma_{1}, \gamma_{2}, \gamma_{2}, \gamma_{1}\right)$. Notice that summing either over all elements which are of the first form or over all elements which are of the second form gives rise to the same series, namely

$$
\eta(\xi)^{2 n} \sum_{\gamma_{1}, \gamma_{2} \in \Gamma} E_{0}\left(n ; \gamma_{1} m, \xi\right) E_{0}\left(n ; \gamma_{2} m, \xi\right)=\left(\sum_{\gamma_{1} \in \Gamma} \eta(\xi)^{n} E_{0}\left(n ; \gamma_{1} m, \xi\right)\right)^{2}=E^{2}(n ; m, \xi) .
$$

However, we cannot just add this series twice because, by doing so, the "diagonal elements", i.e., when $\gamma_{1}=\gamma_{2}=\gamma_{1}^{\prime}=\gamma_{2}^{\prime}$, which belong to both configurations $\left(\gamma_{1}, \gamma_{2}, \gamma_{1}, \gamma_{2}\right)$ and $\left(\gamma_{1}, \gamma_{2}, \gamma_{2}, \gamma_{1}\right)$, would be added twice. Let us for a moment consider the sum without the 
diagonal elements, i.e., let us assume that $\gamma_{1} \neq \gamma_{2}$. Then we have

$$
\begin{aligned}
\sum_{\substack{\gamma \in S_{1} \\
\gamma_{1} \neq \gamma_{2}}} E_{0}\left(n ; \gamma_{1} m, \xi\right) E_{0}\left(n ; \gamma_{2} m, \xi\right) & =\sum_{\gamma_{1}^{\prime}=\gamma_{1} \neq \gamma_{2}=\gamma_{2}^{\prime}} E_{0}\left(n ; \gamma_{1} m, \xi\right) E_{0}\left(n ; \gamma_{2} m, \xi\right) \\
& +\sum_{\substack{\gamma_{2}^{\prime}=\gamma_{1} \neq \gamma_{2}=\gamma_{1}^{\prime} \\
E_{0}}} E_{0}\left(n ; \gamma_{1} m, \xi\right) E_{0}\left(n ; \gamma_{2} m, \xi\right) \\
& =2 \sum_{\gamma_{1} \neq \gamma_{2}} E_{0}\left(n ; \gamma_{1} m, \xi\right) E_{0}\left(n ; \gamma_{2} m, \xi\right) .
\end{aligned}
$$

As for the diagonal elements, the only possibility is $\gamma_{1}=\gamma_{2}=\gamma_{1}^{\prime}=\gamma_{2}^{\prime}$. Hence

$$
\begin{aligned}
\sum_{\substack{\gamma \in S_{1} \\
\gamma_{1}=\gamma_{2}}} E_{0}\left(n ; \gamma_{1} m, \xi\right) E_{0}\left(n ; \gamma_{2} m, \xi\right) & =\sum_{\gamma_{1}=\gamma_{2}=\gamma_{1}^{\prime}=\gamma_{2}^{\prime}} E_{0}\left(n ; \gamma_{1} m, \xi\right) E_{0}\left(n ; \gamma_{2} m, \xi\right) \\
& =\sum_{\gamma_{1} \in \Gamma} E_{0}\left(2 n ; \gamma_{1} m, \xi\right) .
\end{aligned}
$$

Therefore, by splitting the sum over $\gamma_{1}, \gamma_{2}$ as

$$
\begin{aligned}
\sum_{\gamma_{1}, \gamma_{2} \in \Gamma} E_{0}\left(n ; \gamma_{1} m, \xi\right) E_{0}\left(n ; \gamma_{2} m, \xi\right) & =\sum_{\gamma_{1} \neq \gamma_{2}} E_{0}\left(n ; \gamma_{1} m, \xi\right) E_{0}\left(n ; \gamma_{2} m, \xi\right) \\
& +\sum_{\gamma_{1} \in \Gamma} E_{0}\left(2 n ; \gamma_{1} m, \xi\right)
\end{aligned}
$$

we get

$$
\begin{aligned}
& \sum_{\gamma \in S_{1}} E_{0}\left(n ; \gamma_{1} m, \xi\right) E_{0}\left(n ; \gamma_{2} m, \xi\right) \\
& =2 \sum_{\gamma_{1} \neq \gamma_{2}} E_{0}\left(n ; \gamma_{1} m, \xi\right) E_{0}\left(n ; \gamma_{2} m, \xi\right)+\sum_{\gamma_{1} \in \Gamma} E_{0}\left(2 n ; \gamma_{1} m, \xi\right) \\
& =2\left(\sum_{\gamma_{1}, \gamma_{2} \in \Gamma} E_{0}\left(n ; \gamma_{1} m, \xi\right) E_{0}\left(n ; \gamma_{2} m, \xi\right)-\sum_{\gamma_{1} \in \Gamma} E_{0}\left(2 n ; \gamma_{1} m, \xi\right)\right)+\sum_{\gamma_{1} \in \Gamma} E_{0}\left(2 n ; \gamma_{1} m, \xi\right) \\
& =2 \sum_{\gamma_{1}, \gamma_{2} \in \Gamma} E_{0}\left(n ; \gamma_{1} m, \xi\right) E_{0}\left(n ; \gamma_{2} m, \xi\right)-\sum_{\gamma_{1} \in \Gamma} E_{0}\left(2 n ; \gamma_{1} m, \xi\right) .
\end{aligned}
$$

After multiplying the last equation by $\eta(\xi)^{2 n}$, we obtain

$$
f_{4}(m, \xi)=\eta(\xi)^{2 n} \sum_{\gamma \in S_{1}} E_{0}\left(n ; \gamma_{1} m, \xi\right) E_{0}\left(n ; \gamma_{2} m, \xi\right)=2 E^{2}(n ; m, \xi)-E(2 n ; m, \xi)
$$


as required.

\section{General Case}

The general case is treated using the same idea. More precisely, we split the sum over $\gamma_{1}, \ldots, \gamma_{k}$ as a sum of "disjoint" series as in (3.10) and we count how many occurrences of each of those series appear in the sum over $S_{1}$, as is done in (3.11).

Before proceeding further, we need to introduce some notation. A collection $\pi:=$ $\left\{\pi_{1}, \ldots, \pi_{r}\right\}$ of subsets $\pi_{j} \subset \mathbf{k}:=\{1, \ldots, k\}$ is said to be a (set) partition of $\mathbf{k}$ if $\cup_{j=1}^{r} \pi_{j}=\mathbf{k}$ and $\pi_{i} \cap \pi_{j}=\emptyset$ whenever $i \neq j$. Moreover, we will assume (after renaming the indices, if needed) that the blocks $\pi_{j}$ 's of $\pi$ satisfy $\left|\pi_{1}\right| \geq\left|\pi_{2}\right| \geq \cdots \geq\left|\pi_{r}\right|$. It is clear that to each partition $\pi$ corresponds a unique integer partition $\theta(\pi)=\left(\theta_{1}(\pi), \cdots, \theta_{r}(\pi)\right)$ satisfying $\theta_{j}(\pi)=\left|\pi_{j}\right|$ for all $j=1, \ldots, r$.

Let $L_{k}$ be the set of all set partitions of $\mathbf{k}$. We say that $\pi^{(1)} \leq \pi^{(2)}$ if and only if each block of $\pi^{(1)}$ is a subset of a block of $\pi^{(2)}$. It is straightforward to check that with the binary

relation $\leq$, the set $L_{k}$ becomes a lattice. Notice that the least element of $L_{k}$ is the partition $\mathbf{0}_{k}:=\{\{1\}, \ldots,\{k\}\}$ and its greatest element is $\mathbf{1}_{k}:=\{\{1, \ldots, k\}\}$. For more details about set partitions and their relation to integer partitions, the reader is referred to [1].

Every configuration $\gamma \in S_{1}$ can be associated with a unique set partition $\pi(\gamma) \in L_{k}$ (depending exclusively on $\gamma_{1}, \ldots, \gamma_{k}$ ) defined by the condition that two integers $i, j \in \mathbf{k}$ are in the same block if and only if $\gamma_{i}=\gamma_{j}$. Define

$$
\sum_{(\pi)}:=\sum_{\left(\pi_{1}: \cdots: \pi_{r}\right)}:=\sum_{\substack{\gamma_{1}, \ldots, \gamma_{k} \in \Gamma \\ \pi(\gamma)=\pi}}\left(\prod_{l=1}^{k} E_{0}\left(n ; \gamma_{l} m, \xi\right)\right)
$$

where the sum on the right-hand side is over all $\gamma_{1}, \ldots, \gamma_{k} \in \Gamma$ for which some configuration $\gamma=\left(\gamma_{1}, \ldots, \gamma_{k}, \cdot, \ldots, \cdot\right)$ is associated with the partition $\pi$. For example, if $k=7$ and $\pi=\left\{\pi_{1}:=\{1,2,3\}, \pi_{2}:=\{4,5,6\}, \pi_{3}:=\{7\}\right\}$, then

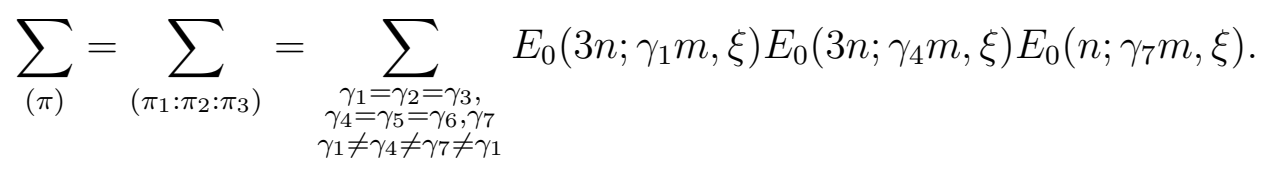


With this notation, we can now decompose the sum over the configurations $\left(\gamma_{1}, \ldots, \gamma_{k}\right)$ according to their underlying set partitions:

$$
\sum_{\gamma_{1}, \ldots, \gamma_{k} \in \Gamma}\left(\prod_{l=1}^{k} E_{0}\left(n ; \gamma_{l} m, \xi\right)\right)=\sum_{\pi \in L_{k}} \sum_{(\pi)}=\sum_{\left(\mathbf{0}_{k}\right)}+\left(\sum_{\mathbf{0}_{k}<\pi<\mathbf{1}_{k}} \sum_{(\pi)}\right)+\sum_{\left(\mathbf{1}_{k}\right)}
$$

Notice that this equation is equal to (3.10) when $k=2$.

We obtain the sum over $S_{1}$ by applying the method we used to derive (3.10) above, i.e., by considering the sums over $S_{1}$ restricted to the different configuration types $(\pi)$ of $\left(\gamma_{1}, \ldots, \gamma_{k}\right)$ appearing on the right-hand side of (3.13). More precisely, we are interested in the sums over the elements of $S_{1}$ restricted to the elements for which the underlying set partition of $\left(\gamma_{1}, \ldots, \gamma_{k}\right)$ corresponds to some $(\pi)$, i.e.,

$$
\sum_{\substack{\gamma \in S_{1}, \pi(\gamma)=\pi}}:=\sum_{\substack{\gamma \in S_{1} \\ \pi(\gamma)=\pi}}\left(\prod_{j=1}^{k} E_{0}\left(n ; \gamma_{j} m, \xi\right)\right)
$$

Since the summands are independent of $\left(\gamma_{1}^{\prime}, \ldots, \gamma_{k}^{\prime}\right)$, it is easy to see that $\sum_{\gamma \in S_{1} ; \pi(\gamma)=\pi}$ is an integer multiple $A_{\pi}$ of $\sum_{(\pi)}$. To be more precise, since for all $i=1, \ldots, k$, there exists $j=1, \ldots, k$ such that $\gamma_{i}=\gamma_{j}^{\prime}$, this multiplicative constant is equal to the number of ways one can arrange the elements $\gamma_{j}^{\prime}$ 's into the $r$ different blocks $\pi_{1}, \ldots, \pi_{r}$ of the partition $\pi$, i.e., $A_{\pi}=\frac{k !}{\theta_{1}(\pi) ! \cdots \theta_{r}(\pi) !}$. Hence

$$
\sum_{\substack{\gamma \in S_{1}, \pi(\gamma)=\pi}}\left(\prod_{j=1}^{k} E_{0}\left(n ; \gamma_{j} m, \xi\right)\right)=\left(\begin{array}{c}
k \\
\theta(\pi)
\end{array}\right) \sum_{(\pi)}
$$

where $\left(\begin{array}{l}k \\ \theta\end{array}\right):=\left(\begin{array}{c}k \\ \theta_{1}, \ldots, \theta_{r}\end{array}\right)=\frac{k !}{\theta_{1} ! \ldots \theta_{r} !}$ is the multinomial coefficient. It follows that

$$
\sum_{\gamma \in S_{1}}=\sum_{\pi \in L_{k}}\left(\begin{array}{c}
k \\
\theta(\pi)
\end{array}\right) \sum_{(\pi)}=k ! \sum_{\left(\mathbf{0}_{k}\right)}+\left(\sum_{\mathbf{0}_{k}<\pi<\mathbf{1}_{k}}\left(\begin{array}{c}
k \\
\theta(\pi)
\end{array}\right) \sum_{(\pi)}\right)+\sum_{\left(\mathbf{1}_{k}\right)} .
$$

Notice that the right-hand side of (3.14) is equal to the right-hand side of (3.11) when $k=2$. 
It remains now to show that the right-hand side of (3.14) is a sum of terms of the form $\prod_{r=1}^{l} E\left(\theta_{r} n ; m, \xi\right)$. Let us write

$$
\sum_{[\pi]}:=\sum_{\left[\pi_{1}: \cdots: \pi_{r}\right]}:=\sum_{\substack{\gamma_{1}, \ldots, \gamma_{k} \in \Gamma \\ \pi(\gamma) \geq \pi}}\left(\prod_{l=1}^{k} E_{0}\left(n ; \gamma_{l} m, \xi\right)\right)
$$

where the sum on the right-hand side is over all $\gamma_{1}, \ldots, \gamma_{k} \in \Gamma$ for which the partition $\pi(\gamma)$ associated with some configuration $\left(\gamma_{1}, \ldots, \gamma_{k}, \cdot, \ldots, \cdot\right)$ satisfies $\pi(\gamma) \geq \pi$. In other words, the sum is over all the elements $\gamma_{1}, \ldots, \gamma_{k} \in \Gamma$ which can be split in the different blocks of $\pi$ according to the following rule: two $\gamma_{j}$ 's in a same block are equal, but two $\gamma_{j}$ 's in different block are not necessarily distinct. For example, if $k=7$ and $\pi=\left\{\pi_{1}:=\{1,2,3\}, \pi_{2}:=\{4,5,6\}, \pi_{3}:=\{7\}\right\}$, then

$$
\sum_{[\pi]}=\sum_{\left[\pi_{1}: \pi_{2}: \pi_{3}\right]}=\sum_{\substack{\gamma_{1}=\gamma_{2}=\gamma_{3}, \gamma_{4}=\gamma_{5}=\gamma_{6}, \gamma_{7}}} E_{0}^{3}\left(n ; \gamma_{1} m, \xi\right) E_{0}^{3}\left(n ; \gamma_{4} m, \xi\right) E_{0}\left(n ; \gamma_{7} m, \xi\right)
$$

Notice that after multiplying the right-hand side by $\eta(\xi)^{7 n}$, it becomes

$$
E(3 n ; m, \xi) E(3 n ; m, \xi) E(n ; m, \xi)
$$

More generally, one always has

$$
\eta(\xi)^{k n} \sum_{\left[\pi_{1}: \cdots: \pi_{r}\right]}=E\left(\theta_{1}(\pi) n ; m, \xi\right) \cdots E\left(\theta_{r}(\pi) n ; m, \xi\right) .
$$

Notice also that $\sum_{\left(\mathbf{1}_{k}\right)}=\sum_{\left[\mathbf{1}_{k}\right]}$.

The idea is now to "convert" the series on the right-hand side of (3.14), which are of the form $\sum_{(\pi)}$, into series of the form $\sum_{[\pi]}$. This would allow us to take advantage of equation (3.15) and then to obtain a sum of elements of the form $\prod_{r=1}^{l} E\left(\theta_{r} n ; m, \xi\right)$. Noticing that the left-hand side of (3.13) is equal to $\sum_{\left[\mathbf{0}_{k}\right]}$, we can apply the idea used to derive (3.13) in order to decompose any sum of the form $\sum_{\left[\pi^{\prime}\right]}$ into sums of the form $\sum_{(\pi)}$ according to the different underlying partitions of the configurations $\left(\gamma_{1}, \ldots, \gamma_{k}\right)$ appearing in $\sum_{\left[\pi^{\prime}\right]}$. This 
gives us a direct generalization of (3.13):

$$
\sum_{\left[\pi^{\prime}\right]}=\sum_{\pi^{\prime} \leq \pi} \sum_{(\pi)}=\sum_{\left(\pi^{\prime}\right)}+\left(\sum_{\pi^{\prime}<\pi<\mathbf{1}_{k}} \sum_{(\pi)}\right)+\sum_{\left[\mathbf{1}_{k}\right]} .
$$

The last equation can be reformulated as

$$
\sum_{\left(\pi^{\prime}\right)}=\sum_{\left[\pi^{\prime}\right]}-\left(\sum_{\pi^{\prime}<\pi} \sum_{(\pi)}\right)
$$

Replacing recursively each $\sum_{\left(\pi^{\prime}\right)}$ in $(3.14)$ by the right-hand side of the previous equation, we can transform the right-hand side of (3.14) into an expression which depends only on sums of the form $\sum_{[\pi]}$. Moreover, since $\sum_{[\pi]}$ depends only on the underlying integer partition of $\pi$, i.e., $\theta(\pi)$, (see, e.g., (3.15)) we can define $\sum_{[\theta]}:=\sum_{[\pi]}$, where $\pi \in L_{k}$ is such that $\theta=\theta(\pi)$. Therefore,

$$
\sum_{\gamma \in S_{1}}=\sum_{l=1}^{k} \sum_{\theta \in \Theta_{l}}\left(C_{\theta} \sum_{[\theta]}\right)
$$

for some integer constants $C_{\theta}$ depending only on the integer partition $\theta$. These coefficients are obtained from the following observation. From (3.16), one can see that the sum $\sum_{[\theta]}$ appears in the expansion (after applying (3.16) recursively) of the sum $\sum_{\left(\pi^{\prime}\right)}$ if and only if there exists $\pi \in L_{k}$ with $\theta(\pi)=\theta$ such that $\pi^{\prime} \leq \pi$. Moreover, the constant in front of the occurrence of $\sum_{[\theta]}$ appearing in the expansion of $\sum_{\left(\pi^{\prime}\right)}$ after applying (3.16) recursively $s$ times corresponds to $(-1)^{s}$ times the number of chains of set partitions of length $s+1$ of the form $\pi^{(s)}:=\pi^{\prime}<\pi^{(s-1)}<\cdots<\pi^{(1)}<\pi$. It follows that

$$
C_{\theta}=\sum_{\substack{\pi \in L_{k}, \theta(\pi)=\theta}}\left(\begin{array}{c}
k \\
\theta(\pi)
\end{array}\right)+\sum_{\substack{\pi^{(s)}<\cdots \pi^{(1)}<\pi, \theta(\pi)=\theta}}(-1)^{s}\left(\begin{array}{c}
k \\
\theta\left(\pi^{(s)}\right)
\end{array}\right) .
$$

where, the second sum on the right-hand side is over all chains (if any) of partitions $\pi^{(s)}<$ $\cdots<\pi^{(1)}<\pi$ of any length $s+1$ satisfying the only condition that $\theta(\pi)=\theta$. For an explanation of how to calculate the coefficients $C_{\theta}$ explicitly, see $\S 3.2$. 


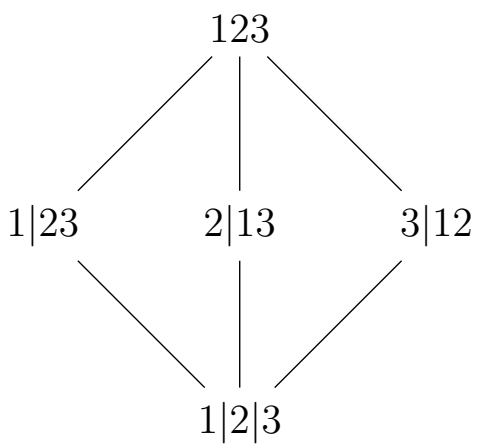

Figure 3-1: The lattice of partitions of a set of 3 elements.

Finally, combining the obtained expression with (3.15), we get

$$
f_{p}(m, \xi):=\eta(\xi)^{k n} \sum_{\gamma \in S_{1}}\left(\prod_{j=1}^{k} E_{0}\left(n ; \gamma_{j} m, \xi\right)\right)=\sum_{l=1}^{k} \sum_{\theta \in \Theta_{l}}\left(C_{\theta} \prod_{r=1}^{l} E\left(\theta_{r} n ; m, \xi\right)\right) .
$$

This concludes the proof of Theorem 1.1.

\subsection{Calculation of the $C_{\theta}$ 's}

Theorem 1.1 provides a full description of the limits of the moments of the Eisenstein series at high-energy. However, when $p$ is even, the limits $f_{p}$ given by (3.17) and (3.18) are not completely explicit in that they depend on a knowledge of the partition lattice $\left(L_{p / 2}, \leq\right)$. An algorithm can be used (at least, when $p$ is small) to determine all the chains of $\left(L_{p / 2}, \leq\right)$. In this section, we illustrate through the examples of $p=6$ and $p=8$ how one can compute $f_{p}$ and the coefficients $C_{\theta}$ from a complete description of $\left(L_{p / 2}, \leq\right)$.

\subsubsection{Example: $p=6$}

A full description of the required set partition lattice $\left(L_{3}, \leq\right)$ is given in Figure $3-1$. The partitions can be classified according to their underlying integer partition: $(1,1,1),(2,1)$ and (3). Therefore, by (3.18),

$$
f_{6}(m)=C_{(1,1,1)} E^{3}(n ; m, \xi)+C_{(2,1)} E(2 n ; m, \xi) E(n ; m, \xi)+C_{(3)} E(3 n ; m, \xi) .
$$


The coefficients $C_{\theta}$ are obtained as follows. For each $\theta$, we list all the chains of the form $\pi^{(s)} \leq \cdots \leq \pi^{(1)} \leq \pi$. For $\theta=(1: 1: 1)$, there is only one chain, namely, $1|2| 3$. Hence

$$
C_{(1,1,1)}=\left(\begin{array}{c}
3 \\
(1,1,1)
\end{array}\right)=6 \text {. }
$$

For $\theta=(2: 1)$, there are three chains of length 1, namely, 1|23, 2|13 and 3|12. Moreover, there are three chains of length $2: 1|2| 3 \leq 1|23,1| 2|3 \leq 2| 13$ and $1|2| 3 \leq 3 \mid 12$. Hence

$$
C_{(2,1)}=3\left(\begin{array}{c}
3 \\
(2,1)
\end{array}\right)-3\left(\begin{array}{c}
3 \\
(1,1,1)
\end{array}\right)=-9 .
$$

For $\theta=(3)$, there is one chain of length 1, i.e., 123, three chains of length 3 , namely, the ones of the form $a|b| c \leq a \mid b c \leq a b c$, and four chains of length 2. The chains of length 2 are of two different types. Three are of the form $a \mid b c \leq a b c$ and the remaining one is $1|2| 3 \leq 123$. Hence

$$
C_{(3)}=\left(\begin{array}{c}
3 \\
(3)
\end{array}\right)-\left(3\left(\begin{array}{c}
3 \\
(2,1)
\end{array}\right)+\left(\begin{array}{c}
3 \\
(1,1,1)
\end{array}\right)\right)+3\left(\begin{array}{c}
3 \\
(1,1,1)
\end{array}\right)=4
$$

Therefore,

$$
f_{6}(m)=6 E^{3}(n ; m, \xi)-9 E(2 n ; m, \xi) E(n ; m, \xi)+4 E(3 n ; m, \xi) .
$$

\subsubsection{Example: $p=8$}

This case is treated using the same approach as above. According to (3.18) and the representation of the lattice $\left(L_{4}, \leq\right)$ given in Figure 3-2, we have

$$
\begin{aligned}
f_{8}(m) & =C_{(1,1,1,1)} E^{4}(n ; m, \xi)+C_{(2,1,1)} E(2 n ; m, \xi) E^{2}(n ; m, \xi)+C_{(2,2)} E^{2}(2 n ; m, \xi) \\
& +C_{(3,1)} E(3 n ; m, \xi) E(n ; m, \xi)+C_{(4)} E(4 n ; m, \xi) .
\end{aligned}
$$

For each $\theta$, we have the following chains:

- $\theta=(1,1,1,1)$ :

* There is one chain of length 1 , namely, $1|2| 3 \mid 4$.

- $\theta=(2,1,1)$ : 


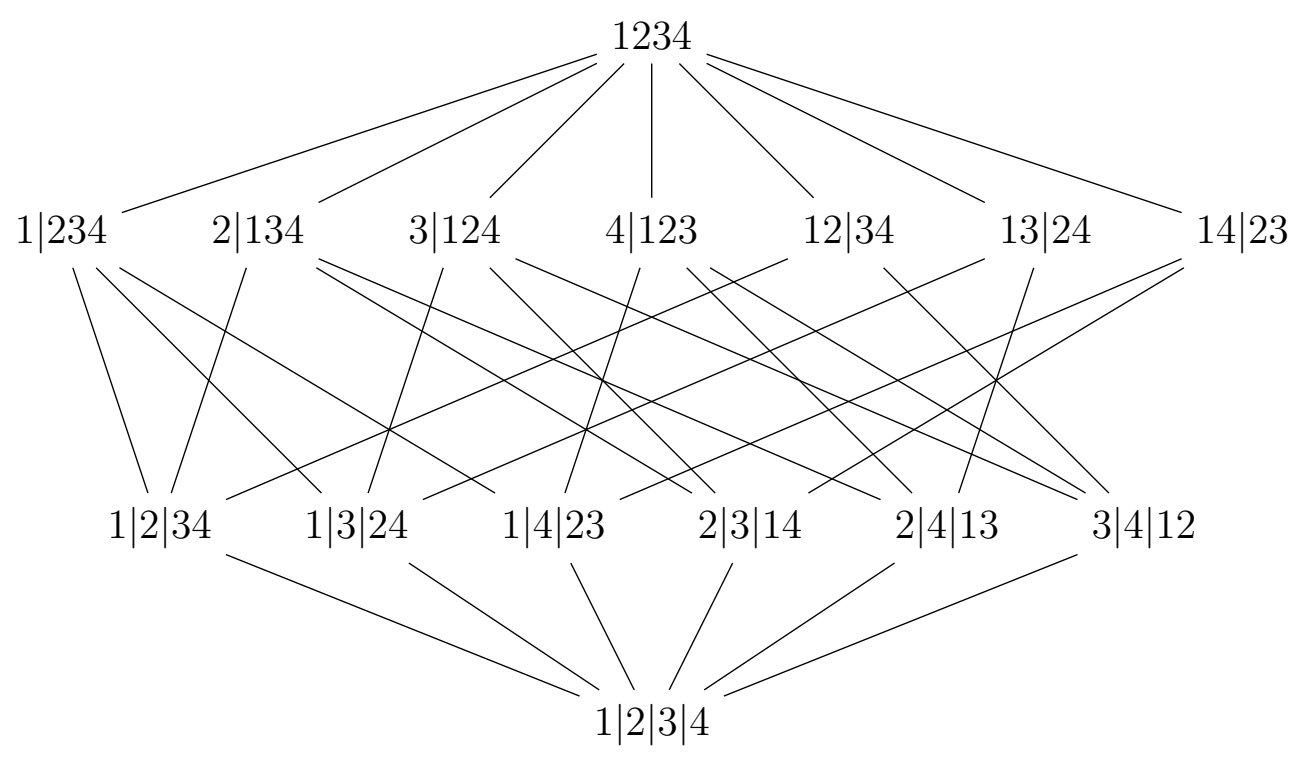

Figure 3-2: The lattice of partitions of a set of 4 elements.

* There are six chains of length 1, i.e., the ones of the form $a|b| c d$;

* There are six chains of length 2, i.e., the ones of the form $a|b| c|d \leq a| b \mid c d$.

- $\theta=(2,2)$ :

* There are three chains of length 1, i.e, the ones of the form $a b \mid c d$;

* There are six chains of length 2 of the form $a|b| c d \leq a b \mid c d$;

* There are three chains of length 2 of the form $a|b| c|d \leq a b| c d$;

* There are six chains of length 3 of the form $a|b| c|d \leq a| b|c d \leq a b| c d$.

- $\theta=(3,1)$ :

* There are four chains of length 1, i.e, the ones of the form $a \mid b c d$;

* There are twelve chains of length 2 of the form $a|b| c d \leq a \mid b c d$;

* There are four chains of length 2 of the form $a|b| c|d \leq a| b c d$;

* There are twelve chains of length 3 of the form $a|b| c|d \leq a| b|c d \leq a| b c d$.

- $\theta=(4)$ :

* There is one chain of length 1, namely, 1234;

* There are four chains of length 2 of the form $a \mid b c d \leq a b c d$;

* There are three chains of length 2 of the form $a b \mid c d \leq a b c d$; 
* There are six chains of length 2 of the form $a|b| c d \leq a b c d$;

* There is one chain of length 2 of the form $a|b| c \mid d \leq a b c d$;

* There are twelve chains of length 3 of the form $a|b| c d \leq a \mid b c d \leq a b c d$;

* There are six chains of length 3 of the form $a|b| c d \leq a b \mid c d \leq a b c d$;

* There are four chains of length 3 of the form $a|b| c|d \leq a| b c d \leq a b c d$;

* There are three chains of length 3 of the form $a|b| c|d \leq a b| c d \leq a b c d$;

* There are six chains of length 3 of the form $a|b| c|d \leq a| b \mid c d \leq a b c d$;

* There are twelve chains of length 4 of the form $a|b| c|d \leq a| b|c d \leq a| b c d \leq a b c d$;

* There are six chains of length 4 of the form $a|b| c|d \leq a| b|c d \leq a b| c d \leq a b c d$. Therefore, the coefficients $C_{\theta}$ are given by

$$
\begin{aligned}
& C_{(1,1,1,1)}=\left(\begin{array}{c}
4 \\
(1,1,1,1)
\end{array}\right)=24, \\
& C_{(2,1,1)}=6\left(\begin{array}{c}
4 \\
(2,1,1)
\end{array}\right)-6\left(\begin{array}{c}
4 \\
(1,1,1,1)
\end{array}\right)=-72, \\
& C_{(2,2)}=3\left(\begin{array}{c}
4 \\
(2,2)
\end{array}\right)-\left(6\left(\begin{array}{c}
4 \\
(2,1,1)
\end{array}\right)+3\left(\begin{array}{c}
4 \\
(1,1,1,1)
\end{array}\right)\right)+6\left(\begin{array}{c}
4 \\
(1,1,1,1)
\end{array}\right)=18, \\
& C_{(3,1)}=4\left(\begin{array}{c}
4 \\
(3,1)
\end{array}\right)-\left(12\left(\begin{array}{c}
4 \\
(2,1,1)
\end{array}\right)+4\left(\begin{array}{c}
4 \\
(1,1,1,1)
\end{array}\right)\right)+12\left(\begin{array}{c}
4 \\
(1,1,1,1)
\end{array}\right)=64 \text {, } \\
& C_{(4)}=\left(\begin{array}{c}
4 \\
(4)
\end{array}\right)-\left(4\left(\begin{array}{c}
4 \\
(3,1)
\end{array}\right)+3\left(\begin{array}{c}
4 \\
(2,2)
\end{array}\right)+6\left(\begin{array}{c}
4 \\
(2,1,1)
\end{array}\right)+\left(\begin{array}{c}
4 \\
(1,1,1,1)
\end{array}\right)\right) \\
& +\left(18\left(\begin{array}{c}
4 \\
(2,1,1)
\end{array}\right)+13\left(\begin{array}{c}
4 \\
(1,1,1,1)
\end{array}\right)\right)-18\left(\begin{array}{c}
4 \\
(1,1,1,1)
\end{array}\right)=-33 .
\end{aligned}
$$

Hence

$$
\begin{aligned}
f_{8}(m) & =24 E^{4}(n ; m, \xi)-72 E(2 n ; m, \xi) E^{2}(n ; m, \xi)+18 E^{2}(2 n ; m, \xi) \\
& +64 E(3 n ; m, \xi) E(n ; m, \xi)-33 E(4 n ; m, \xi) .
\end{aligned}
$$




\subsubsection{The hyperbolic space $X=\mathbb{H}^{n+1}$}

When $\Gamma$ is the trivial group, the manifold $X$ is the $(n+1)$-dimensional hyperbolic space and

$$
E\left(s_{\lambda} ; m, \xi\right)=E_{0}\left(s_{\lambda} ; m, \xi\right)=E_{0}\left(\frac{n}{2} ; m, \xi\right) E_{0}^{i \lambda}(1 ; m, \xi)
$$

For $k=\frac{p}{2}$, the integral $I_{k}^{p}(\lambda, \xi)$ then takes the following simple form:

$$
I_{p / 2}^{p}(\lambda, \xi)=\int_{X} a(m) E\left(\frac{p}{2} n ; m, \xi\right) d v(m),
$$

which is independent of $\lambda$. In other words, in the special case where $X=\mathbb{H}^{n+1}$,

$$
I_{p / 2}^{p}(\lambda, \xi) \longrightarrow \int_{X} a(m) E\left(\frac{p}{2} n ; m, \xi\right) d v(m) \quad \text { as } \lambda \longrightarrow \infty
$$

Notice that the coefficients of the terms appearing in the expressions of $f_{2}, f_{4}, f_{6}$ and $f_{8}$ given above (see (3.9), (3.12), (3.19) and (3.20) respectively) all sum up to 1 . This observation is in concordance with the fact that when $X=\mathbb{H}^{n+1}$, all the terms are the same and the coefficient of this term should be equal to 1 according to (3.21).

\subsection{The moments of $F_{\lambda}$}

We conclude this chapter with a calculation of the moments of the real and imaginary parts of the Eisenstein series, when seen as random variables over a compact subset of $X$, as explained in $\S 1.1$. In the case where $p$ is odd, since the limits as $\lambda \rightarrow \infty$ of $I_{k}^{p}(\lambda, \xi)$ given by Theorem 1.1 are 0 for all $0 \leq k \leq p$, it is clear from the binomial expansion of $F_{\lambda}^{p}$ (see (1.3) above), that

$$
\mathbb{E}\left(F_{\lambda}^{p}(\cdot, \xi)\right) \longrightarrow 0
$$

as $\lambda \rightarrow \infty$. These limits appeared already in the statement of Corollary 1.4, in which a remainder is also provided.

On the other hand, when $p$ is even, the integral $I_{p / 2}^{p}(\lambda, \xi)$, which corresponds to the "middle-term" in the binomial expansion of $F_{\lambda}^{p}$ does not vanish. The high-energy limit 
of $\mathbb{E}\left(F_{\lambda}^{p}(\cdot, \xi)\right)$ is then equal to the limit as $\lambda \rightarrow \infty$ of $I_{p / 2}^{p}(\lambda, \xi)$ times the coefficient of $\left|E\left(s_{\lambda} ; m, \xi\right)\right|^{p}$ in the expansion of $F_{\lambda}^{p}$, namely

$$
\frac{1}{2^{p}}\left(\begin{array}{l}
p \\
\frac{p}{2}
\end{array}\right)
$$

Therefore, we have the following corollary as a direct consequence of Theorem 1.1.

Corollary 3.3. Under the hypothesis of Theorem 1.1, if $p$ is even, then

$$
\mathbb{E}\left(F_{\lambda}^{p}(\cdot, \xi)\right) \longrightarrow \int_{X} a(m)\left(\frac{1}{2^{p}}\left(\begin{array}{c}
p \\
\frac{p}{2}
\end{array}\right) f_{p}(m, \xi)\right) d v_{X}(m)
$$

as $\lambda \rightarrow \infty$, where $f_{p}$ is given by (3.18). In particular,

$$
\begin{gathered}
\frac{1}{2^{2}}\left(\begin{array}{l}
2 \\
1
\end{array}\right) f_{2}(m, \xi)=\frac{1}{2} E(n ; m, \xi), \\
\frac{1}{2^{4}}\left(\begin{array}{l}
4 \\
2
\end{array}\right) f_{4}(m, \xi)=\frac{3}{4} E^{2}(n ; m, \xi)-\frac{3}{8} E(2 n ; m, \xi), \\
\frac{1}{2^{6}}\left(\begin{array}{l}
6 \\
3
\end{array}\right) f_{6}(m, \xi)=\frac{15}{8} E^{3}(n ; m, \xi)-\frac{45}{16} E(2 n ; m, \xi) E(n ; m, \xi)+\frac{5}{4} E(3 n ; m, \xi),
\end{gathered}
$$

and

$$
\begin{aligned}
\frac{1}{2^{8}}\left(\begin{array}{l}
8 \\
4
\end{array}\right) f_{8}(m, \xi) & =\frac{105}{16} E^{4}(n ; m, \xi)-\frac{315}{16} E(2 n ; m, \xi) E^{2}(n ; m, \xi)+\frac{315}{64} E^{2}(2 n ; m, \xi) \\
& +\frac{35}{2} E(3 n ; m, \xi) E(n ; m, \xi)-\frac{1155}{128} E(4 n ; m, \xi) .
\end{aligned}
$$




\section{CHAPTER 4}

\section{An error term for the odd-order moments}

With this chapter we begin our investigation of the rate of vanishing of the odd-order moments of Eisenstein series at high-energy. For now, we restrict ourselves to the case where $p$ is odd. In $\S 4.1$, we give the proof of Theorem 1.2, which provides a polynomial remainder term for the limit as $\lambda \rightarrow \infty$ of integrals of the form $I_{k}^{p}(\lambda, \xi)$. The following section is concerned with improving this error term in the special case where $X$ is a surface and $p=3$. Moreover, the approach introduced there will be exploited again when dealing with the fourth moment in the next chapter.

\subsection{Proof of Theorem $\mathbf{1 . 2}$}

As in the proof of Theorem 1.1, we work in a fixed fundamental domain $\mathcal{F}$ of $\Gamma$ in $\mathbb{H}^{n+1}$. In view of $(3.2)$, the $p$-fold $\gamma=\left(\gamma_{1}, \ldots, \gamma_{p}\right) \in \underbrace{\Gamma \times \cdots \times \Gamma}_{p \text { times }}$ will also be seen as a point in the $p$-times Cartesian product of $S^{n}=\partial \mathbb{B}^{n+1}$ as follows:

$$
\gamma=\left(\gamma_{1}^{-1} \xi, \ldots, \gamma_{p}^{-1} \xi\right) \in \mathbb{S}^{p}:=\underbrace{S^{n} \times \cdots \times S^{n}}_{p \text { times }} .
$$

Unless explicitly stated otherwise, the remaining non-standard notation used in this section is the one introduced in $\S 3.1$.

\section{Outline of the proof}

It is clear from the proof of Theorem 1.1 that it is sufficient to show that

$$
\sum_{\gamma \in S} I_{\gamma}(\lambda)=\mathcal{O}\left(\lambda^{-\frac{1}{d}}\right)
$$

as $\lambda \rightarrow \infty$. The proof then amounts to showing that for every fixed $\gamma$, the oscillatory integral $I_{\gamma}(\lambda)$ is $\mathcal{O}_{\gamma}\left(\lambda^{-\frac{1}{d}}\right)$ as $\lambda \rightarrow \infty$, and that the implied constant (depending on $\gamma$ ) decreases fast enough as $d\left(0, \gamma_{l} 0\right) \rightarrow \infty, l=1, \ldots, p$, so that they sum up to a finite limit over all the 
elements of the group. More precisely, we proceed in three steps. We will first show that the points at which the phase is stationary correspond to the zero set of a polynomial in several variables that can be contained in a compact hypersurface of $\mathbb{B}^{n+1}$, the $n$-dimensional volume of which will be bounded uniformly with respect to $\gamma$. By controlling the rate at which the gradient of the phase vanishes on this hypersurface, we will then be able to apply tools from the theory of oscillatory integrals to obtain the desired bound on $I_{\gamma}$. Finally, using classic

results of Patterson and Sullivan about the exponent of convergence of the Poincaré series and the Hausdorff dimension of the limit set, we will conclude that these bounds sum up to a finite limit over the elements of the group.

\subsubsection{Finding the zero set of $\nabla_{m} \psi_{\gamma}$}

By the proof of Lemma 3.1, we already know that the stationary points of $\psi_{\gamma}$ are contained in an algebraic variety of codimension at least 1 . However, this information alone appears to be insufficient to control the growth of $\left|I_{\gamma}(\lambda)\right|$ with respect to $\gamma$. We will then try to acquire a more precise knowledge of this zero set by analyzing the phase function in more detail.

By (3.2) and (3.3), we have

$$
\nabla_{m} \psi_{\gamma}(m)=\sum_{j=1}^{k} \nabla_{m} \phi_{\xi_{j}}(m)-\sum_{j=1}^{p-k} \nabla_{m} \phi_{\xi_{j+k}}(m)=\frac{-2 G_{\gamma}(m)}{\left(1-|m|^{2}\right) \prod_{l=1}^{p}\left|m-\xi_{l}\right|^{2}}
$$

where

$$
\begin{aligned}
G_{\gamma}(m) & :=(2 k-p) m \prod_{l=1}^{p}\left|m-\xi_{l}\right|^{2} \\
& +\left(1-|m|^{2}\right)\left[\sum_{j=1}^{k}\left(m-\xi_{j}\right) \prod_{l \neq j}\left|m-\xi_{l}\right|^{2}-\sum_{j=k+1}^{p}\left(m-\xi_{j}\right) \prod_{l \neq j}\left|m-\xi_{l}\right|^{2}\right] .
\end{aligned}
$$

Since supp $a$ is compact, the denominator of the fraction on the right-hand side of (4.1) is bounded from below uniformly for $\gamma \in \mathbb{S}^{p}$ and $m \in \operatorname{supp} a$. Therefore, $\nabla_{m} \phi_{\xi}$ vanishes exactly where $G_{\gamma}$ does. For $m=\left(x_{1}, \ldots, x_{n+1}\right) \in \mathcal{F}$, it is easy to see that each of the $n+1$ components of the vector-valued function $G_{\gamma}(m)$ is a polynomial in the variables $x_{1}, \ldots, x_{n+1}$ with coefficients depending continuously on $\gamma$. One then has that $\left|G_{\gamma}(m)\right|^{2}$ is 
a real polynomial of degree $d \leq 4 p+2$, in the single variable $x:=x_{1}$, with coefficients $A_{j}(\gamma, y)$ 's that are continuous functions of the variables $\gamma \in \mathbb{S}^{p}$ and $y:=\left(x_{2}, \ldots, x_{n+1}\right)$ :

$$
\left|G_{\gamma}(m)\right|^{2}=A_{d}(\gamma, y) x^{d}+A_{d-1}(\gamma, y) x^{d-1}+\cdots+A_{1}(\gamma, y) x+A_{0}(\gamma, y) .
$$

In particular, if $k=0$ or $p$, one can check from (4.2) that the leading term of the polynomial corresponding to the $x$-component cancels. Thus $d \leq 4 p$ in these two cases.

As noted in $\S 2.2 .4$, the hyperbolic gradient of the Busemann function, i.e., $\tilde{\nabla}_{m} \phi_{\xi}(m):=$ $\frac{1-|m|^{2}}{2} \nabla \phi_{\xi}(m)$, corresponds to the Euclidean unit vector pointing in the direction of the geodesic joining $m \in \mathbb{B}^{n+1}$ to $\xi$. It follows that the vector $\tilde{\nabla}_{m} \phi_{\xi}(m)$ tends to a unit vector perpendicular to $S^{n}$ whenever $m \rightarrow \eta \in S^{n}$. The definition of $\tilde{\nabla}_{m} \phi_{\xi}(m)$ can then be extended continuously to $\overline{\mathbb{B}^{n+1}}$. Notice that $\tilde{\nabla}_{m} \phi_{\xi}(m)$ can be defined in $\mathbb{R}^{n+1}-\overline{\mathbb{B}^{n+1}}$ as well by (3.3). Moreover, one can see from (3.3) that, by doing so, we obtain a function $\tilde{\nabla}_{m} \phi_{\xi}(m)$ continuous everywhere in $\mathbb{R}^{n+1}$ and such that $\left|\tilde{\nabla}_{m} \phi_{\xi}(m)\right|=1$ on $S^{n}$.

Therefore, $\tilde{\nabla}_{m} \psi_{\gamma}(m)$ tends to the sum of $p$ parallel unit vectors whenever $\mathbb{R}^{n+1} \ni m \rightarrow$ $\eta \in S^{n}$. Since $p$ is odd, the modulus of the resulting vector must be a positive integer. It follows that for any $\eta \in S^{n}$, there exists a neighbourhood of $\eta$ in $\mathbb{R}^{n+1}$ such that $\tilde{\nabla}_{m} \psi_{\gamma}(m)>0$ in this neighbourhood. Since the denominator of the right-hand side of (4.1) does not vanish outside of $S^{n}$, it is impossible for $\max _{j}\left|A_{j}(\gamma, y)\right|$ to be equal to zero for any $\gamma \in \mathbb{S}^{p}$ and $y \in \mathbb{R}^{n}$. Indeed, this would imply that $\tilde{\nabla}_{m} \psi_{\gamma}(m)$ vanishes on the line parametrized by $x \mapsto(x, y)$ with $(x, y) \in \mathbb{R}^{n+1}-S^{n+1}$, which would be a contradiction to the observation above. The continuous function $\max _{j=1, \ldots, d}\left|A_{j}(\gamma, y)\right|: \mathbb{S}^{p} \times \mathbb{R}^{n} \rightarrow \mathbb{R}$ can then be minimized on the compact subset $\mathbb{S}^{p} \times \overline{B_{0}^{n}(1)}$ by some positive constant $C_{\min }$.

For $1 \leq s \leq d$, define $G_{s}(\gamma, y, x):=\sum_{k=0}^{s} A_{k}(\gamma, y) x^{k}$. By the Fundamental Theorem of Algebra, this polynomial can be factored as follows when $(\gamma, y) \notin A_{s}^{-1}\{0\}$ :

$$
G_{s}(\gamma, y, x)=A_{s}(\gamma, y) \sum_{k=0}^{s} \frac{A_{k}(\gamma, y)}{A_{s}(\gamma, y)} x^{k}=A_{s}(\gamma, y)\left(x-C_{1, s}(\gamma, y)\right) \cdots\left(x-C_{s, s}(\gamma, y)\right)
$$


where $C_{j, s}(\gamma, y)$ 's are continuous functions of the coefficients $A_{k-1}(\gamma, y) / A_{s}(\gamma, y)$ for $k=$ $1, \ldots, s$. Let $\left(\gamma_{0}, y_{0}\right) \in A_{s}^{-1}\{0\}$ and let $z_{j}\left(\gamma_{0}, y_{0}\right), j=1, \ldots, s$, denote the zeros of the polynomial $G_{s}\left(\gamma_{0}, y_{0}, x\right)$. By Theorem 1 in [44], for all $\epsilon>0$, there exists a number $\delta_{\epsilon}\left(\gamma_{0}, y_{0}\right)>0$ such that for a fixed $j$, either $\left|C_{j, s}(\gamma, y)-z_{j}\left(\gamma_{0}, y_{0}\right)\right|<\epsilon$ or $\left|C_{j, s}(\gamma, y)\right|>1 / \epsilon$ whenever $\left|A_{k}(\gamma, y)-A_{k}\left(\gamma_{0}, y_{0}\right)\right|<\delta_{\epsilon}\left(\gamma_{0}, y_{0}\right)$ for all $k=1, \ldots, s$. In other words, when $(\gamma, y) \rightarrow\left(\gamma_{0}, y_{0}\right)$, either $C_{j, s}(\gamma, y)$ converges to some complex numbers, in which case the function $C_{j, s}(\gamma, y)$ can be continually extended at $\left(\gamma_{0}, y_{0}\right)$, or $C_{j, s}(\gamma, y)$ diverges to infinity. Extend $C_{j, s}(\gamma, y)$ continually where possible and let $F_{j, s}$ denote the subset of $A_{s}^{-1}\{0\} \cap\left(\mathbb{S}^{p} \times \overline{B_{0}^{n}(1)}\right)$ for which $C_{j, s}(\gamma, y)$ diverges to infinity as $(\gamma, y)$ approaches it.

The proof of Theorem 1 in [44] also shows that $\delta_{\epsilon}(\gamma, y)$ depends continuously on the coefficients $A_{k}(\gamma, y)$ and, consequently, also on $\gamma$ and $y$. Therefore, if we set $\epsilon:=1$ in the statement, we can minimize the continuous function $\delta_{1}(\gamma, y)$ over the compact subset $A_{s}^{-1}\{0\} \cap\left(\mathbb{S}^{p} \times \overline{B_{0}^{n}(1)}\right)$ of $\mathbb{R}^{p+n}$ by some positive real number $\delta_{\min }$. Since $\delta_{\min }>0$ and the functions $A_{k}(\gamma, y)$ are uniformly continuous on $\mathbb{S}^{p} \times \overline{B_{0}^{n}(1)}$, the existence of a uniform $\delta_{0}>0$ such that $\left|C_{j}(\gamma, y)\right|>1$ whenever dist $\left((\gamma, y), F_{j, s}\right)<\delta_{0}$ is guaranteed. Therefore,

$$
S_{s, \gamma}:=\left(\bigcup_{j=1}^{s}\left\{\left(\operatorname{Re}\left(C_{j}(\gamma, y)\right), y\right): y \in \overline{B_{0}^{n}(1)}, \operatorname{dist}\left((\gamma, y), F_{j, s}\right) \geq \delta_{0}\right\}\right) \cap \operatorname{supp} a
$$

contains all zeros of $(x, y) \mapsto G_{s}(\gamma, y, x)$ in supp a. Moreover, it is constructed as the intersection with supp $a$ of a finite union of submanifolds of $\mathbb{R}^{n+1}$ of codimension at least 1 .

If $m_{0}=\left(x_{0}, y_{0}\right)$ is a zero of $\nabla_{m} \psi_{\gamma}$, then by (4.1), $m_{0}$ must also be a zero of $\left|G_{\gamma}(m)\right|^{2}$. It follows from the definition of $G_{s}(\gamma, y, x)$ that $G_{s_{0}}\left(\gamma, y_{0}, x_{0}\right)=0$ for some $0<s_{0} \leq d$ with $A_{s_{0}}\left(\gamma, y_{0}\right) \neq 0$. Therefore, the construction above shows that $S_{\gamma}:=\bigcup_{s=1}^{d} S_{s, \gamma}$ contains all zeros of the map $\left(m_{0}\right) \mapsto \nabla_{m} \psi_{\gamma}\left(m_{0}\right)$ in supp $a$. Moreover, $S_{\gamma}$ corresponds to the intersection with supp $a$ of a finite union of submanifolds of $\mathbb{R}^{n+1}$ of codimension at least 1 .

\subsubsection{Bounding $I_{\gamma}$}

Now that we know where the stationary points of the phase are localized, we can apply the method of stationary phase to $I_{\gamma}$. More precisely, we will integrate by parts outside of 
the submanifold $S_{\gamma}$ that we found in the previous subsection. This will be done through the use of an appropriate smooth cutoff function.

Let $\Omega_{\gamma}^{\lambda}:=\left\{m \in \mathbb{B}^{n+1}: \operatorname{dist}\left(m, S_{\gamma}\right)<\frac{1}{\lambda}\right\}$. Since $S_{\gamma}$ is a compact subset of $\mathbb{B}^{n+1}$, there exists a function $\rho_{\gamma}^{\lambda}(m) \in C^{\infty}\left(\mathbb{B}^{n+1}\right)$ satisfying the following properties:

(i) $0 \leq \rho_{\gamma}^{\lambda}(m) \leq 1$

(ii) $\rho_{\gamma}^{\lambda}(m)=0$ if $m \in \Omega_{\gamma}^{2 \lambda}$;

(iii) $\rho_{\gamma}^{\lambda}(m)=1$ if $m \notin \Omega_{\gamma}^{\lambda}$;

(iv) $\left|\partial_{j} \rho_{\gamma}^{\lambda}(m)\right| \leq C \lambda \chi_{\overline{\Omega_{\gamma}^{\lambda}} \backslash \Omega_{\gamma}^{2 \lambda}}(m)$ for a constant $C$ independent of $\gamma$ and $\lambda$.

Indeed, define

$$
\eta_{\lambda}(m):= \begin{cases}\lambda^{n+1} \exp \left(\frac{1}{|2 \lambda m|^{2}-1}\right), & \text { if }|m|<\frac{1}{2 \lambda} \\ 0 & \text { if }|m| \geq \frac{1}{2 \lambda}\end{cases}
$$

This function is (up to a multiplicative constant) the standard mollifier for the ball $B\left(0, \frac{1}{2 \lambda}\right)$. It is well-known that the function $\rho_{\gamma}^{\lambda}:=1-\eta_{\lambda} * \chi_{\Omega_{\gamma}^{2 \lambda}}$ is $C^{\infty}\left(\mathbb{B}^{n+1}\right)$ and satisfies properties (i), (ii) and (iii) above. As regards property (iv), since $\rho_{\gamma}^{\lambda}$ is $C^{\infty}\left(\mathbb{B}^{n+1}\right)$ and constant everywhere but in $\Omega_{\gamma}^{\lambda} \backslash \Omega_{\gamma}^{2 \lambda}$, its derivatives can be bounded by a constant $C_{\gamma, \lambda}$ times the characteristic function $\chi_{\overline{\Omega_{\gamma}^{\lambda}} \backslash \Omega_{\gamma}^{2 \lambda}}$. Then, it remains only to show that this constant can be written in the form $C_{\gamma, \lambda}=C \lambda$. Since $\chi_{\Omega_{\gamma}^{2 \lambda}}$ has compact support in $\mathbb{R}^{n+1}$, we have

$$
\begin{aligned}
\left|\partial_{j} \rho_{\gamma}^{\lambda}(m)\right| & =\left|\int_{\mathbb{B}^{n+1}} \partial_{j} \eta_{\lambda}(m-w) \chi_{\Omega_{\gamma}^{2 \lambda}}(w) d w\right| \\
& =\left|\int_{\mathbb{B}^{n+1} \cap\left\{|m-w|<\frac{1}{2 \lambda}\right\}} \lambda^{n+1} \frac{8 \lambda^{2}\left(m_{j}-w_{j}\right)}{\left(|2 \lambda(m-w)|^{2}-1\right)^{2}} \exp \left(\frac{1}{|2 \lambda(m-w)|^{2}-1}\right) \chi_{\Omega_{\gamma}^{2 \lambda}}(w) d w\right| \\
& \leq \lambda \int_{\mathbb{B}^{n+1} \cap\left\{|m-w|<\frac{1}{2}\right\}} \frac{8\left|m_{j}-w_{j}\right|}{\left(|2(m-w)|^{2}-1\right)^{2}} \exp \left(\frac{1}{|2(m-w)|^{2}-1}\right) d w \leq C \lambda
\end{aligned}
$$

for all $m \in \operatorname{supp} a$. Hence $\left|\partial_{j} \rho_{\gamma}^{\lambda}(m)\right| \leq C \lambda \chi_{\overline{\Omega_{\gamma}^{\lambda}} \backslash \Omega_{\gamma}^{2 \lambda}}(m)$.

We can now split the integrand of $I_{\gamma}(\lambda)$ into two parts: one being defined in a neighbourhood of the zero set of $\nabla_{m} \psi_{\gamma}$ and one having its support away from $S_{\gamma}$. In order to simplify 
the notation, we will write $E_{0}^{\gamma}(m)$ as a shorthand for $E_{0}(1 ; \gamma m, \xi)$. Let

$$
I_{\gamma}(\lambda)=A_{\gamma}(\lambda)+B_{\gamma}(\lambda)
$$

where

$$
A_{\gamma}(\lambda):=\int_{\mathbb{H}^{n+1}} a(m)\left(1-\rho_{\gamma}^{\lambda^{\beta}}(m)\right)\left(\prod_{l=1}^{p} E_{0}^{\gamma_{l}}(m)\right)^{\frac{n}{2}} e^{i \lambda \psi_{\gamma}(m)} \frac{2^{n+1} d m}{\left(1-|m|^{2}\right)^{n+1}}
$$

and

$$
B_{\gamma}(\lambda):=\int_{\mathbb{H}^{n+1}} a(m) \rho_{\gamma}^{\lambda^{\beta}}(m)\left(\prod_{l=1}^{p} E_{0}^{\gamma_{l}}(m)\right)^{\frac{n}{2}} e^{i \lambda \psi_{\gamma}(m)} \frac{2^{n+1} d m}{\left(1-|m|^{2}\right)^{n+1}},
$$

for some positive $\beta \in \mathbb{R}$ that will be fixed later and which will only depend on $p$ and $n$.

Keeping in mind that we will eventually sum the integrals over the elements of the group $\Gamma$, we have to pay particular attention to the way $I_{\gamma}$ depends on $\gamma \in \mathbb{S}^{p}$. A factor which may affect strongly the value of $I_{\gamma}$ is the "quantity" of stationary points. For this reason, it is important to make sure that the effect of $\gamma$ on the "size" of the set $S_{\gamma}$ can be controlled in a favorable way.

Let $\operatorname{Vol}_{n}(\Omega)$ denote the $n$-dimensional Riemannian volume of a measurable subset $\Omega \subset \mathbb{R}^{n}$ (with the convention that $\operatorname{Vol}_{n}(\Omega)=0$ if $\operatorname{dim}(\Omega) \neq n$ ). Notice that with this notation $\operatorname{Vol}_{k}\left(S_{\gamma}\right)$ is finite for all $0 \leq k \leq n$. Indeed, this is a direct consequence of our construction of $S_{\gamma}$ as the intersection of a finite union of submanifolds of $\mathbb{R}^{n+1}$ with the compact subset supp $a$.

By Weyl's results in [43], for any $\gamma$, the volume of the tubular region $\Omega_{\gamma}^{\lambda}$ is equal to a polynomial of degree $\tilde{n} \leq n+1$ in the variable $\lambda^{-1}$, where the coefficients of the terms of degree less than the codimension of $S_{\gamma}$ are 0 and the other coefficients are integral invariants of the finitely-many submanifolds forming $S_{\gamma}$. In particular, the coefficient of the term of degree 0 is always zero, and the first non-zero term is the volume of the corresponding submanifold. Moreover, since $\left(\operatorname{Re}\left(C_{j, s}(\gamma, y)\right), y\right)$ is a continuous function of $\gamma$, those coefficients vary continuously with respect to $\gamma \in \mathbb{S}^{p}$. We can then bound them from above by some 
positive constants $C_{k}$ independent of $\gamma, k=1, \ldots, \tilde{n}$. Hence, there exists a positive constant $C$ independent of $\gamma$ such that

$$
\operatorname{Vol}_{n+1}\left(\Omega_{\gamma}^{\lambda}\right) \leq \sum_{k=1}^{\tilde{n}} C_{k} \lambda^{-k} \leq C \lambda^{-1}
$$

when $\lambda$ is large.

Lemma 2.19 and Property (i) of the function $\rho_{\gamma}^{\lambda}$ that we constructed above allow us to bound the integrand of $A_{\gamma}(\lambda)$. We can then use the estimate on the size of the support of this integrand given by (4.5) to obtain an upper bound for the integral. Namely,

$$
\left|A_{\gamma}(\lambda)\right| \leq C \lambda^{-\beta} \prod_{l=1}^{p} e^{-\frac{n}{2} d\left(0, \gamma_{l} 0\right)}
$$

when $\lambda$ is large.

On the other hand, since the zero set of $\nabla_{m} \psi_{\gamma}$ is outside of the support of $\rho_{\gamma}^{\lambda^{\beta}}$ for all $\lambda$, we may apply integration by parts to $B_{\gamma}(\lambda)$ :

$$
B_{\gamma}(\lambda)=\frac{1}{i \lambda} \int_{\mathbb{H}^{n+1}} e^{i \lambda \psi_{\gamma}(m)} \nabla^{*}\left(a(m) \rho_{\gamma}^{\lambda^{\beta}}(m)\left(\prod_{l=1}^{p} E_{0}^{\gamma_{l}}(m)\right)^{\frac{n}{2}} \frac{\nabla_{m} \psi_{\gamma}(m)}{\left|\nabla_{m} \psi_{\gamma}(m)\right|^{2}}\right) \frac{2^{n+1} d m}{\left(1-|m|^{2}\right)^{n+1}},
$$

where $\nabla^{*}$ is the adjoint of $\nabla$ with respect to the volume measure $\frac{2^{n+1} d m}{\left(1-|m|^{2}\right)^{n+1}}$. We would like to have a bound of the form (4.6) for $B_{\gamma}(\lambda)$, so that it can be summed over all the elements of the group. In order to achieve this, we will consider $B_{\gamma}(\lambda)$ as an iterated integral in Cartesian coordinates, first integrating with respect to the variable $x$. This inside integral will be shown to be bounded by a function independent of $y$. The compactness of supp $a$ will then allow us to neglect integration with respect to $y$ and to conclude that this function is essentially the bound that we are looking for. Let

$$
J_{\gamma}(y, \lambda):=\int_{-\infty}^{\infty} e^{i \lambda \psi_{\gamma}(m)} \nabla^{*}\left(a(m) \rho_{\gamma}^{\lambda^{\beta}}(m)\left(\prod_{l=1}^{p} E_{0}^{\gamma_{l}}(m)\right)^{\frac{n}{2}} \frac{\nabla_{m} \psi_{\gamma}(m)}{\left|\nabla_{m} \psi_{\gamma}(m)\right|^{2}}\right) \frac{2^{n+1} d x}{\left(1-|m|^{2}\right)^{n+1}}
$$


Since the integrand of $B_{\gamma}$ is $C_{c}^{\infty}\left(\mathbb{B}^{n+1}\right)$, Fubini's theorem applies to give

$$
B_{\gamma}(\lambda)=\frac{1}{i \lambda} \int_{B_{0}^{n}(r)} J_{\gamma}(y, \lambda) d y
$$

where $0<r<1$ is large enough so that $\operatorname{supp} a \subset B_{0}^{n+1}(r)$.

Since $\left|\nabla_{m} \psi_{\gamma}(m)\right|$ may become very small when $m$ is close to $S_{\gamma}$, we need to control the rate at which $\left|\nabla_{m} \psi_{\gamma}(m)\right|$ decreases as $m$ approaches a zero of $\nabla_{m} \psi_{\gamma}$. We claim that for any $(\gamma, y)$, there exist $1 \leq s_{\gamma, y} \leq d$, a set of not necessarily distinct complex numbers $Z_{y, \gamma}:=\left\{Z_{1}, \ldots, Z_{s_{\gamma, y}}\right\}$, and a constant $C>0$ depending only on $p$, supp $a$ and $C_{\min }$, such that

$$
\left|\nabla_{m} \psi_{\gamma}(m)\right|^{2} \geq C\left|x-Z_{1}\right| \cdots\left|x-Z_{s_{\gamma, y}}\right|
$$

whenever $m \in \operatorname{supp} a$. Moreover, $Z_{j}$ are such that $\left(Z_{j}, y\right)$ is a zero of $\nabla_{m} \psi_{\gamma}$. Note that the set $Z_{y, \gamma}$ is possibly empty. If this is the case, then the inequality above is understood as $\left|\nabla_{m} \psi_{\gamma}(m)\right|^{2} \geq C$. Note also that the complex numbers $Z_{j}$ 's depend on $(y, \gamma)$. The reference to $(y, \gamma)$ was omitted in the name of the variable only to alleviate the notation.

We proceed now with the proof of this claim. Fix $(\gamma, y)$ and let $s \leq d$ be the largest integer such that $A_{s}(\gamma, y)$ in (4.3) is different from 0 . If $s=0$, then $Z_{y, \gamma}$ is empty and the claim follows trivially by setting $C:=C_{\min }$. Otherwise, the function $\left|G_{\gamma}(m)\right|^{2}$ can be expressed as $A_{s}(\gamma, y)$ times a polynomial in the variable $x$ with coefficients $\left(A_{j} / A_{s}\right)(\gamma, y)$ and roots $C_{j, s}(\gamma, y)$ as in (4.4). It is well-known that those coefficients can be expressed as symmetric polynomials of the roots $C_{j, s}$ 's in the following way:

$$
\frac{A_{k-1}}{A_{s}}=(-1)^{k} e_{s-k+1}\left(C_{1, s}, \ldots, C_{s, s}\right)
$$

where $e_{i}\left(X_{1}, \ldots, X_{n}\right)=\sum_{1 \leq j_{1}<j_{2}<\cdots<j_{i} \leq n} X_{j_{1}} \cdots X_{j_{i}}$. It follows that

$$
\left|\frac{A_{k-1}}{A_{s}}\right| \leq K_{p} \max _{1 \leq j_{1}<j_{2}<\cdots<j_{s-k+1} \leq s}\left|C_{j_{1}, s}\right| \cdots\left|C_{j_{s-k+1}, s}\right|,
$$


for some positive constant $K_{p}$ depending only on $p$. Assuming temporarily that $\left|A_{s}\right|<\frac{C_{\min }}{K_{p}}$ and combining this with that last inequality gives

$$
K_{p}<\frac{C_{\min }}{\left|A_{s}\right|} \leq \max _{k}\left|\frac{A_{k-1}}{A_{s}}\right| \leq K_{p}\left|C_{j_{1}, s}\right| \cdots\left|C_{j_{l}, s}\right|
$$

for some $l$ and $1 \leq j_{1}<\cdots<j_{l} \leq s$. Since, by the inequalities above $\left|C_{j_{1}, s}\right| \cdots\left|C_{j_{l}, s}\right|>1$, there exists a non-empty subset of $1 \leq j_{1}<\cdots<j_{l} \leq s$ for which $\left|C_{j_{i}, s}\right| \geq 1$. By relabelling this subset $C_{1, s}, \ldots, C_{l, s}$ if needed (the new $l$ being less than or equal to the former one), we can then assume that (4.9) holds with $\left|C_{i, s}\right| \geq 1$. Define

$$
\alpha_{i}:=\frac{\log \frac{\left(\left|A_{s}\right|\left|C_{1, s}\right| \cdots\left|C_{l, s}\right|\right)^{\frac{1}{l}}}{\left|C_{i, s}\right|}}{\log \left|A_{s}\right|} .
$$

A direct consequence of this definition is that $\alpha_{1}+\cdots+\alpha_{l}=1$ and

$$
\left|A_{s}\right|^{\alpha_{i}}\left|C_{i, s}\right|=\left(\left|A_{s}\right|\left|C_{1, s}\right| \cdots\left|C_{l, s}\right|\right)^{\frac{1}{l}} \geq\left(C_{\min } / K_{p}\right)^{1 / l}
$$

The last inequality follows from (4.9). Hence, for $m \in \operatorname{supp} a$,

$$
\left|A_{s}\right|^{\alpha_{i}}\left|C_{i, s}-x\right| \geq\left|A_{s}\right|^{\alpha_{i}}\left|C_{i, s}\right|\left(1-\frac{|x|}{\left|C_{i, s}\right|}\right) \geq\left(\frac{r^{l} C_{\min }}{K_{p}}\right)^{\frac{1}{l}}
$$

where $r:=\operatorname{dist}\left(\partial \mathbb{B}^{n+1}, \operatorname{supp} a\right)>0$ since supp $a$ is compact. The last inequality follows from (4.10) and the fact that $\left|C_{i, s}\right| \geq 1$. By (4.4) and (4.11),

$$
\begin{aligned}
\left|G_{\gamma}(m)\right|^{2} & =\left(\left|A_{s}\right|^{\alpha_{1}}\left|x-C_{1, s}\right|\right) \cdots\left(\left|A_{s}\right|^{\alpha_{l}}\left|x-C_{l, s}\right|\right)\left|x-C_{l+1, s}\right| \cdots\left|x-C_{s, s}\right| \\
& \geq \frac{r^{l} C_{\min }}{K_{p}}\left|x-C_{l+1, s}\right| \cdots\left|x-C_{s, s}\right| \geq \frac{r^{d} C_{\min }}{K_{p}}\left|x-C_{l+1, s}\right| \cdots\left|x-C_{s, s}\right| .
\end{aligned}
$$

The claim (4.8) follows then from (4.1) by setting $s_{\gamma, y}:=s-l, Z_{j}:=C_{l+j, s}$ and $C:=\frac{r^{d} C_{\min }}{2^{p-2} K_{p}}$. Notice that since $|r|<1$, our additional assumption that $\left|A_{s}\right|<\frac{C_{\min }}{K_{p}}$ can automatically be removed. Indeed, if $\left|A_{s}\right| \geq \frac{C_{\min }}{K_{p}}$, the statement of the claim is trivially satisfied with $C$ defined as above and $Z_{j}:=C_{j, s}\left(\gamma_{0}, y\right)$. 
We can now use the claim that was just proven to find an appropriate upper bound for $J_{\gamma}(y, \lambda)$. If $Z_{y, \gamma}$ is empty, the integrand of $J_{\gamma}(y, \lambda)$ can be bounded from above by a constant independent of $x$. Therefore, in this case, applying Lemma 2.19, we obtain

$$
\left|J_{\gamma}(y, \lambda)\right| \leq C \prod_{l=1}^{p} e^{-\frac{n}{2} d\left(0, \gamma_{l} 0\right)}
$$

On the other hand, if $Z_{y, \gamma}$ is not empty, the term $\frac{\nabla_{m} \psi_{\gamma}(m)}{\left|\nabla_{m} \psi_{\gamma}(m)\right|^{2}}$ and its derivatives, which appear in the integrand of $J_{\gamma}(y, \lambda)$, are not bounded from above uniformly with respect to $x$. We have

$$
\left|\sum_{j=1}^{n+1} \partial_{j} \frac{\partial_{j} \psi_{\gamma}}{\left|\nabla_{m} \psi_{\gamma}\right|^{2}}\right| \leq \sum_{j=1}^{n+1}\left[\frac{\left|\partial_{j}^{2} \psi_{\gamma}\right|}{\left|\nabla_{m} \psi_{\gamma}\right|^{2}}+2 \sum_{k=1}^{n+1} \frac{\left|\partial_{j} \psi_{\gamma}\right|\left|\partial_{k} \psi_{\gamma}\right|\left|\partial_{j} \partial_{k} \psi_{\gamma}\right|}{\left|\nabla_{m} \psi_{\gamma}\right|^{4}}\right] \leq \frac{C^{\prime}}{\left|\nabla_{m} \psi_{\gamma}\right|^{2}}
$$

for some constant $C^{\prime}$ depending only on $n, p, \xi$ and supp $a$. Notice that the last inequality follows from direct calculations via (3.3) and (4.1) of the derivatives of $\nabla_{m} \psi_{\gamma}$. The claim above can now be applied to bound $\left|\nabla_{m} \psi_{\gamma}\right|$ from below by (4.8). By doing this, (4.13) can be bounded further by an expression which only depends on $x$. Namely,

$$
\left|\sum_{j=1}^{n+1} \partial_{j} \frac{\partial_{j} \psi_{\gamma}}{\left|\nabla_{m} \psi_{\gamma}\right|^{2}}\right| \leq \frac{C}{\left(\left(x-Z_{1}\right) \cdots\left(x-Z_{s_{\gamma, y}}\right)\right)}
$$

where $C$ is a positive constant depending only on $n, p, \xi$ and supp $a$. Therefore,

$$
\left|J_{\gamma}(y, \lambda)\right| \leq\left(\prod_{l=1}^{p} e^{-\frac{n}{2} d\left(0, \gamma_{l} 0\right)}\right)\left[\left(C_{1} \lambda^{\beta}+C_{2}\right) J_{\gamma, \frac{1}{2}}(y, \lambda)+C_{3} J_{\gamma, 1}(y, \lambda)\right]
$$

where

$$
J_{\gamma, \alpha}(y, \lambda):=\int_{-1}^{1} \frac{\left(1-\chi_{\Omega_{\gamma}^{2 \lambda^{\beta}}}(x, y)\right) d x}{\left|\left(x-Z_{1}\right) \cdots\left(x-Z_{s_{\gamma, y}}\right)\right|^{\alpha}} .
$$

Note that we have made use of Lemma 2.19 and properties (ii) and (iv) of the cut-off function $\rho_{\gamma}^{\lambda}$

The integrals $J_{\gamma, \alpha}(y, \lambda)$ 's appearing on the right-hand side of (4.15) are tractable enough to derive from them the bound of the form (4.6) that we are looking for. By relabelling and taking a subset if needed, we may assume that $-1 \leq \operatorname{Re}\left(Z_{1}\right) \leq \cdots \leq \operatorname{Re}\left(Z_{s_{\gamma}, y}\right) \leq 1$. If 
$\operatorname{Re}\left(Z_{i}\right) \neq \operatorname{Re}\left(Z_{i+1}\right)$ let $q_{i}$ be the midpoint of the interval $\left[\operatorname{Re}\left(Z_{i}\right), \operatorname{Re}\left(Z_{i+1}\right)\right]$. Notice that

$$
\frac{1}{\left|\left(x-Z_{1}\right) \cdots\left(x-Z_{s_{\gamma, y}}\right)\right|} \leq \begin{cases}\frac{2^{d}}{\left|x-\operatorname{Re}\left(Z_{1}\right)\right|^{d}}, & \text { if } x \in\left(-1, Z_{1}\right] \\ \frac{2^{d}}{\left|x-\operatorname{Re}\left(Z_{i}\right)\right|^{d}}, & \text { if } x \in\left[q_{i-1}, q_{i}\right], \quad i=2, \ldots, s_{\gamma, y}-1 \\ \frac{2^{d}}{\left|x-\operatorname{Re}\left(Z_{s_{\gamma, y}}\right)\right|^{d}}, & \text { if } x \in\left[Z_{s_{\gamma, y}}, 1\right) .\end{cases}
$$

Therefore, assuming that $d$ is maximal, i.e., either $4 p+2$ or $4 p$ depending on whether $0<k<p$, or $k=0, p$ respectively, there exist $K, K^{\prime}>0$ such that

$$
\begin{aligned}
\frac{J_{\gamma, \alpha}(y, \lambda)}{2^{d}} & \leq \int_{-1}^{\operatorname{Re}\left(Z_{1}\right)} \frac{\left(1-\chi_{\Omega_{\gamma}^{2 \lambda^{\beta}}}(x, y)\right) d x}{\left|x-\operatorname{Re}\left(Z_{1}\right)\right|^{\alpha d}}+\sum_{i=2}^{s_{\gamma, y}-1} \int_{q_{i-1}}^{q_{i}} \frac{\left(1-\chi_{\Omega_{\gamma}^{2 \lambda^{\beta}}}(x, y)\right) d x}{\left|x-\operatorname{Re}\left(Z_{i}\right)\right|^{\alpha d}} \\
& +\int_{\operatorname{Re}\left(Z_{s_{\gamma, y}}\right)}^{1} \frac{\left(1-\chi_{\Omega_{\gamma}^{2 \lambda^{\beta}}}(x, y)\right) d x}{\left|x-\operatorname{Re}\left(Z_{s_{\gamma, y}}\right)\right|^{\alpha d}} \\
& \leq K \int_{(2 \lambda)-\beta}^{2} \frac{d x}{x^{\alpha d}}=K^{\prime} \lambda^{\beta(\alpha d-1)}
\end{aligned}
$$

for large values of $\lambda$. Using this estimate in conjunction with (4.7), (4.12) and (4.15), we can now bound the integral of $B_{\gamma}(\lambda)$ :

$$
\left|B_{\gamma}(\lambda)\right| \leq C^{\prime}\left(\lambda^{-1}+\lambda^{\beta(d / 2)-1}+\lambda^{\beta(d / 2-1)-1}+\lambda^{\beta(d-1)-1}\right) \prod_{l=1}^{p} e^{-\frac{n}{2} d\left(0, \gamma_{l} 0\right)},
$$

when $\lambda$ is large.

It remains only to fix the undetermined positive constant $\beta$ appearing in the expressions of the upper bounds of $A_{\gamma}(\lambda)$ and $B_{\gamma}(\lambda)$. We want to choose it so that the exponent of $\lambda$ be the optimal one in view of (4.6) and (4.16). One can check that this happens when $\beta:=\frac{1}{d}$. Using the fact that $d-1 \geq \frac{d}{2} \geq \frac{d}{2}-1 \geq 0$ when $d \geq 2$, we derive the bound

$$
\left|B_{\gamma}(\lambda)\right| \leq C \lambda^{-\frac{1}{d}} \prod_{l=1}^{p} e^{-\frac{n}{2} d\left(0, \gamma_{l} 0\right)}
$$

from (4.16). Combining it with (4.6), we obtain

$$
\left|I_{\gamma}(\lambda)\right| \leq C \lambda^{-\frac{1}{d}} \prod_{l=1}^{p} e^{-\frac{n}{2} d\left(0, \gamma_{l} 0\right)}
$$

when $\lambda$ is large. 


\subsubsection{Summing over the elements of $\Gamma$}

By (2.11) and Theorem 2.15, since $\delta_{\Gamma}<\frac{n}{2}$, we have

$$
\sum_{\gamma_{1}, \ldots, \gamma_{p} \in \Gamma} \prod_{l=1}^{p} e^{-\frac{n}{2} d\left(0, \gamma_{l} 0\right)}=\prod_{l=1}^{p}\left(\sum_{\gamma_{l} \in \Gamma} e^{-\frac{n}{2} d\left(0, \gamma_{l} 0\right)}\right)<\infty
$$

It follows from (4.17) that

$$
\sum_{\gamma_{1}, \ldots, \gamma_{p} \in \Gamma} I_{\gamma}(\lambda)=\mathcal{O}\left(\lambda^{-\frac{1}{d}}\right)
$$

as $\lambda \rightarrow \infty$.

The remainder $\mathcal{O}\left(\lambda^{-\frac{1}{d}}\right)$ depends a priori on the point $\xi \in \partial \bar{X}$. By looking carefully at the proof, one notices that the dependence on $\xi$ comes only from the constant introduced by the application of Lemma 2.19. However, as noted in Remark 2.7, since $\xi$ lifts on the universal cover to a point in $\overline{\mathcal{F}} \cap S^{n}$, this constant can be chosen to be independent of $\xi$.

Therefore, the implied constant of $\mathcal{O}\left(\lambda^{-\frac{1}{d}}\right)$ depends only on $k, p$ and the function $a$. This concludes the proof of Theorem 1.2.

\subsection{An improved error term for the third moment on surfaces}

In this section and in the next chapter, we restrict our attention to convex co-compact quotients of the hyperbolic plane, i.e., to $X=\Gamma \backslash \mathbb{H}^{n+1}$, when $n=1$. Working with surfaces will allow us to perform explicit calculations that would not be possible (or at least, would not be as easy to carry out) on manifolds of higher dimensions.

As can be seen in the proof of Theorem 1.2, the rate of vanishing of the odd-order moments depends considerably on the degree of the zeros of $\nabla_{m} \psi_{\gamma}(m)$; see $(4.1)$ in $\S 4$. In the general case, when no specific information about the roots of $\nabla_{m} \psi_{\gamma}(m)$ is available, one has to deal with the worst-case scenario, i.e., when the zeros are of maximal degree, which is what we do in the proof of Theorem 1.2. On the other hand, if one has an explicit expression for $\nabla_{m} \psi_{\gamma}(m)$ at their disposal, then it might be easier to study the roots. One can thus expect a significantly faster convergence. 
Here we take advantage of the simplicity of the expression of $\psi_{\gamma}$ when $n=1$ and $p=3$ to obtain the same limit as in Theorem 1.2, but with a faster rate of convergence. Note that Theorem 1.2 gives a bound of the order of $\lambda^{-\frac{1}{14}}$ in this case.

Proposition 4.1. Let $X=\Gamma \backslash \mathbb{H}^{2}$ be a convex co-compact hyperbolic surface with $\delta_{\Gamma}<1 / 2$. Let $a \in C_{0}^{\infty}(X)$ and $E(s ; \cdot, \xi)$ be an Eisenstein series with $\xi \in \partial \bar{X}$. Then for any $0 \leq k \leq 3$,

$$
\int_{X} a(m)\left(E\left(s_{\lambda} ; m, \xi\right)\right)^{k}\left(\overline{E\left(s_{\lambda} ; m, \xi\right)}\right)^{3-k} d v_{X}(m)=\mathcal{O}\left(\lambda^{-1}\right)
$$

as $\lambda \rightarrow \infty$. Moreover, the implied constant in the bound $\mathcal{O}\left(\lambda^{-1}\right)$ is independent of $\xi \in \partial \bar{X}$.

The proof of this proposition follows the general scheme introduced in the previous section. More precisely, after writing the integral of the third power of the Eisenstein series as a series over the elements of the group $\Gamma \times \Gamma \times \Gamma$, we find the points at which the phases of the integrals $I_{\gamma}$ in the summation are stationary. We then apply the usual stationary phase method in a way that allows us to control the growth of the integrals $I_{\gamma}$ as $\gamma$ varies over $\mathbb{S}^{3}$. Finally, we apply an argument similar to the one described in $\S 4.1 .3$ to sum those integrals over $\Gamma \times \Gamma \times \Gamma$. However, the proof of Proposition 4.1 differs essentially from the one described in $\S 4$ in the way the integrals $I_{\gamma}$ are bounded. Instead of analyzing the zero set of $\nabla_{m} \psi_{\gamma}(m)$ for all the possible configurations $\gamma=\left(\gamma_{1}^{-1} \xi, \gamma_{2}^{-1} \xi, \gamma_{3}^{-1} \xi\right) \in \mathbb{S}^{3}$, we fix a point $\xi_{0}:=\left(\xi_{1}, \xi_{2}, \xi_{3}\right) \in \mathbb{S}^{3}$ for which the calculations involving $\nabla_{m} \psi_{\xi_{0}}$ are convenient, and we reduce the treatment of the configurations $\gamma$ to the one of $\xi_{0}$ via an isometry of $\mathbb{H}^{2}$. Such a simplified approach is possible only because the specific nature of the problem provides us with a precise knowledge of the stationary points. The proof of Theorem 1.3 in the next chapter is also partly based on this idea.

With this procedure in mind, the question of controlling the growth of $I_{\gamma}$ with respect to $\gamma \in \mathbb{S}^{3}$ boils down to understanding how the integral $I_{\gamma}$ is affected when the three boundary points $\gamma_{1}^{-1} \xi, \gamma_{2}^{-1} \xi$ and $\gamma_{3}^{-1} \xi$ associated with $\gamma \in \mathbb{S}^{3}$ are displaced by an isometry $\gamma_{0}$ of $\mathbb{H}^{2}$. The results about Möbius transformations stated and proved in $§ 2.1 .1$ will reveal useful to this end. 


\subsubsection{Proof of Proposition 4.1}

Since we will work on surfaces from now on, we will use the notation $\mathbb{D}:=\mathbb{B}^{2} \subset \mathbb{C}$ for the Poincaré unit disk. Otherwise, we keep using the notation introduced previously. We treat three cases separately, depending on the value of $k$.

Case 1: $k=3$

We suppose first that $\gamma_{1}^{-1} \xi, \gamma_{2}^{-1} \xi$ and $\gamma_{3}^{-1} \xi$ are all different. In order to apply tools from the theory of oscillatory integrals, we need first to know for which points $m \in \mathbb{D}$ the equation $\nabla_{m} \psi_{\gamma}(m)=0$ holds, where $\nabla_{m}$ is the Euclidean gradient. Since the hyperbolic gradient $\tilde{\nabla}_{m}$ satisfies $\tilde{\nabla}_{m}=\frac{1-|m|^{2}}{2} \nabla_{m}$, we have $\nabla_{m} \psi(m)=0$ in $\mathbb{D}$ if and only if $\tilde{\nabla}_{m} \psi(m)=0$. Moreover, as noted in $\S 2.2 .4$, the hyperbolic gradient of the Busemann function $\phi_{\xi}(m)$ is the unit vector pointing in the direction of the geodesic from $m$ to $\xi$. Since $\tilde{\nabla}_{m} \psi_{\gamma}=\sum_{k=1}^{3} \tilde{\nabla} \phi_{\gamma_{1}^{-1} \xi}$, finding the zeros of $\tilde{\nabla}_{m} \psi_{\gamma}(m)$ boils down to finding the points $m$ in $\mathbb{D}$ for which the sum of the three unit vectors pointing respectively in the directions of the geodesics joining $m$ to $\gamma_{1}^{-1} \xi, \gamma_{2}^{-1} \xi$ and $\gamma_{3}^{-1} \xi$ vanishes.

As noted at the beginning of the section, the proof consists mainly in applying isometries of $\mathbb{H}^{2}$ to reduce the calculations of $\tilde{\nabla}_{m} \psi_{\gamma}$ to the one of $\tilde{\nabla}_{m} \psi_{\xi_{0}}$ for a carefully chosen point $\xi_{0}=\left(\xi_{1}, \xi_{2}, \xi_{3}\right) \in \mathbb{S}^{3}$. Define $\xi_{1}:=i, \xi_{2}:=\frac{\sqrt{3}}{2}-\frac{1}{2} i$ and $\xi_{3}:=-\frac{\sqrt{3}}{2}-\frac{1}{2} i$. The three points form an equilateral ideal triangle. By geometric considerations, it is easy to see that the sum of the three unit vectors $\tilde{\nabla}_{m} \phi_{\xi_{1}}(m), \tilde{\nabla} \phi_{\xi_{2}}(m)$ and $\tilde{\nabla} \phi_{\xi_{3}}(m)$ vanishes if and only if $m=0$. Let $\gamma \in \mathrm{PSL}_{2}(\mathbb{R})$ be such that $\xi_{j}=\gamma \gamma_{j}^{-1} \xi$ (after a reordering of $\xi_{j}$ if needed).

We are interested in the limit as $\lambda \rightarrow \infty$ of $\sum_{\gamma_{1}, \gamma_{2}, \gamma_{3} \in \Gamma} I_{\gamma}(\lambda)$. Since the hyperbolic measure is invariant under $\operatorname{Isom}_{+}\left(\mathbb{H}^{n+1}\right)$ (this follows, e.g., from Lemma 2.4 and the usual formula for change of variables under integration), we have

$$
I_{\gamma}(\lambda)=\int_{\mathbb{D}} a\left(\gamma^{-1} m\right)\left(\prod_{l=1}^{3} E_{0}^{\gamma_{l} \gamma^{-1}}(m)\right)^{\frac{n}{2}} e^{i \lambda \psi_{\gamma}\left(\gamma^{-1} m\right)} \frac{4 d m}{\left(1-|m|^{2}\right)^{2}}
$$

Notice that the integrand is zero outside of $\gamma(\operatorname{supp} a)$. 
We want to apply the method of stationary phase to the integral $I_{\gamma}$. Keeping in mind that this procedure involves integrating by parts outside of the stationary points, we have first to determine precisely the zero set of the gradient of the phase function. By Lemma 2.18

$$
\begin{aligned}
\psi_{\gamma}\left(\gamma^{-1} m\right) & =\sum_{j=1}^{3}\left[\phi_{\gamma_{j}^{-1} \xi}\left(\gamma^{-1} m\right)+\log \left|D \gamma_{j}\left(\gamma_{j}^{-1} \xi\right)\right|\right] \\
& =\sum_{j=1}^{3}\left[\phi_{\gamma \gamma_{j}^{-1} \xi}(m)+\log \left|D \gamma^{-1}\left(\gamma \gamma_{j}^{-1} \xi\right)\right|+\log \left|D \gamma_{j}\left(\gamma_{j}^{-1} \xi\right)\right|\right]
\end{aligned}
$$

The only terms depending on $m$ on the right-hand side of this equation are the functions $\phi_{\gamma \gamma_{j}^{-1} \xi}(m)=\phi_{\xi_{j}}(m)$. Hence

$$
\nabla_{m} \psi_{\gamma}\left(\gamma^{-1} m\right)=\sum_{j=1}^{3} \nabla_{m} \phi_{\xi_{j}}(m)
$$

A general formula for the gradient of the Busemann function was already calculated in (3.3). We can use this expression with $\xi_{1}, \xi_{2}$ and $\xi_{3}$ to write $\nabla_{m} \psi_{\gamma}\left(\gamma^{-1} m\right)$ via (4.19) as a rational function in terms of $m$ and its complex conjugate $\bar{m}$ :

$$
\nabla_{m} \psi_{\gamma}\left(\gamma^{-1} m\right)=\nabla_{m} \phi_{\xi_{1}}(m)+\nabla_{m} \phi_{\xi_{2}}(m)+\nabla_{m} \phi_{\xi_{3}}(m)=\frac{6\left(\bar{m}^{2}-i m\right)}{\left(|m|^{2}-1\right)\left(\bar{m}^{3}-i\right)}
$$

where $\bar{m}$ denotes the complex conjugate of $m$. This expression alone is sufficient to conclude that the unique stationary point of $I_{\gamma}$ is 0 . However, keeping in mind that our goal is to carry out an integration by parts around this point, we need an appropriate lower bound for $\nabla_{m} \psi_{\gamma}\left(\gamma^{-1} m\right)$. Since $\frac{\bar{m}-i m / \bar{m}}{\bar{m}^{3}-i}$ is bounded away from 0 when $m \in \mathbb{D}$, we have

$$
\left|\nabla_{m} \psi_{\gamma}\left(\gamma^{-1} m\right)\right| \geq \frac{C|m|}{1-|m|^{2}}
$$

for some constant $C>0$.

Knowing that the phase of the integral above is stationary only at 0 , we can apply the method of stationary phase by decomposing $I_{\gamma}$ as the sum of two integrals, one inside and one outside of a neighbourhood of the origin. Define $S_{\gamma}:=\{m=0\}$ and let $\rho_{\gamma}^{\lambda} \in C^{\infty}(\mathbb{D})$ 
and $\Omega_{\gamma}^{\lambda}$ be as in $\S 4$ 1.2. Let $\beta$ be a positive real number that will be fixed later and define

$$
A_{\gamma}(\lambda):=\int_{\mathbb{D}}\left(1-\rho_{\gamma}^{\lambda^{\beta}}(m)\right) a\left(\gamma^{-1} m\right)\left(\prod_{l=1}^{3} E_{0}^{\gamma l \gamma^{-1}}(m)\right)^{\frac{1}{2}} e^{i \lambda \psi_{\gamma}\left(\gamma^{-1} m\right)} \frac{4 d m}{\left(1-|m|^{2}\right)^{2}}
$$

and

$$
B_{\gamma}(\lambda):=\int_{\mathbb{D}} \rho_{\gamma}^{\lambda^{\beta}}(m) a\left(\gamma^{-1} m\right)\left(\prod_{l=1}^{3} E_{0}^{\gamma_{l} \gamma^{-1}}(m)\right)^{\frac{1}{2}} e^{i \lambda \psi_{\gamma}\left(\gamma^{-1} m\right)} \frac{4 d m}{\left(1-|m|^{2}\right)^{2}}
$$

Notice that $I_{\gamma}(\lambda)=A_{\gamma}(\lambda)+B_{\gamma}(\lambda)$. By the Weyl's tube formula [43] and the discussion leading to (4.5) above, the Euclidean volume of $\Omega_{\gamma}^{\lambda}$ satisfies $\operatorname{Vol}_{n+1}\left(\Omega_{\gamma}^{\lambda}\right) \leq C \lambda^{-2}$, for some constant $C>0$ independent of $\gamma$. Therefore, we deduce from Lemma 2.19 that

$$
\left|A_{\gamma}(\lambda)\right| \leq C \lambda^{-2 \beta} \prod_{l=1}^{3} e^{-\frac{1}{2} d\left(0, \gamma_{l} 0\right)}
$$

when $\lambda$ is large.

On the other hand, the respective upper bound for the integral $B_{\gamma}(\lambda)$ will be obtained through multiple integrations by parts. By (4.21), the zero set of $\nabla_{m} \psi_{\gamma}\left(\gamma^{-1} m\right)$ is contained outside of the support of $\rho_{\gamma}$. We may then apply integration by parts $j$ times to $B_{\gamma}(\lambda)$ :

$$
\begin{aligned}
B_{\gamma}(\lambda) & =\frac{4}{(i \lambda)^{j}} \int_{\mathbb{D}} \nabla^{*}\left(\frac { \nabla _ { m } \psi _ { \gamma } ( \gamma ^ { - 1 } m ) } { | \nabla _ { m } \psi _ { \gamma } ( \gamma ^ { - 1 } m ) | ^ { 2 } } \nabla ^ { * } \left(\cdots \nabla ^ { * } \left(\frac{\nabla_{m} \psi_{\gamma}\left(\gamma^{-1} m\right)}{\left|\nabla_{m} \psi_{\gamma}\left(\gamma^{-1} m\right)\right|^{2}}\right.\right.\right. \\
& \left.\left.\left.\times \nabla^{*}\left(\rho_{\gamma}^{\lambda^{\beta}}(m) u_{\gamma}(m) \frac{\nabla_{m} \psi_{\gamma}\left(\gamma^{-1} m\right)}{\left|\nabla_{m} \psi_{\gamma}\left(\gamma^{-1} m\right)\right|^{2}}\right)\right) \cdots\right)\right) e^{i \lambda \psi_{\gamma}\left(\gamma^{-1} m\right)} d m
\end{aligned}
$$

where $\nabla^{*}$ is the adjoint of $\nabla$ with respect to the measure $d m$, and

$$
u_{\gamma}(m):=\frac{a\left(\gamma^{-1} m\right)}{\left(1-|m|^{2}\right)^{2}}\left(\prod_{l=1}^{3} E_{0}^{\gamma_{l} \gamma^{-1}}(m)\right)^{\frac{1}{2}}
$$

By Lemma 2.19 and Lemma 2.5, we have

$$
\left|\partial_{\alpha} u_{\gamma}(m)\right| \leq \frac{C \prod_{l=1}^{3} e^{-\frac{1}{2} d\left(0, \gamma_{l} 0\right)}}{\left(1-|m|^{2}\right)^{2+|\alpha|}}
$$


for $m \in \gamma(\operatorname{supp} a)$. On the other hand, since $\left|\nabla_{m} \psi_{\gamma}\left(\gamma^{-1} m\right)\right|^{2}$ is a homogeneous polynomial of degree 2 in $\partial_{i} \psi_{\gamma}\left(\gamma^{-1} m\right)$, a straightforward inductive argument gives us that

$$
\partial_{\alpha} \frac{\partial_{j} \psi_{\gamma}\left(\gamma^{-1} m\right)}{\left|\nabla_{m} \psi_{\gamma}\left(\gamma^{-1} m\right)\right|^{2}}=\frac{Q_{2^{|\alpha|+1}+|\alpha|-1}^{\left(2^{|\alpha|+1}-|\alpha|-1\right)}\left(\partial_{1} \psi_{\gamma}\left(\gamma^{-1} m\right), \partial_{2} \psi_{\gamma}\left(\gamma^{-1} m\right)\right)}{\left|\nabla_{m} \psi_{\gamma}\left(\gamma^{-1} m\right)\right|^{2^{|\alpha|+1}}}
$$

where $Q_{l}^{(k)}\left(X_{1}, X_{2}\right)$ is a homogeneous polynomial of degree $k$ with coefficients being integer multiples of products of higher partial derivatives of $\psi_{\gamma}\left(\gamma^{-1} m\right)$ such that the total order for each term is $l$; see (4.13) as an example for the case where $|\alpha|=1$. Applying Lemma 2.5 to these coefficients, i.e., with $F(m):=\partial_{j} \psi_{\gamma}(m)$, and the trivial inequality $\left|\partial_{j} \psi_{\gamma}\left(\gamma^{-1} m\right)\right| \leq$ $\left|\nabla_{m} \psi_{\gamma}\left(\gamma^{-1} m\right)\right|$ to the "variables" $X_{j}=\partial_{j} \psi_{\gamma}\left(\gamma^{-1} m\right)$ of the polynomial $Q_{2^{|\alpha|+1}+|\alpha|-1}^{\left(2^{|\alpha|+1}-|\alpha|-1\right)}$ in $(4.25)$, we get

$$
\left|\partial_{\alpha} \frac{\partial_{j} \psi_{\gamma}\left(\gamma^{-1} m\right)}{\left|\nabla_{m} \psi_{\gamma}\left(\gamma^{-1} m\right)\right|^{2}}\right| \leq \frac{C_{\alpha}}{\left(1-|m|^{2}\right)^{2|\alpha|}\left|\nabla_{m} \psi_{\gamma}\left(\gamma^{-1} m\right)\right|^{|\alpha|+1}} \leq \frac{C_{\alpha}}{|m|^{|\alpha|+1}\left(1-|m|^{2}\right)^{|\alpha|-1}},
$$

for some $C_{\alpha}>0$, when $m \in \gamma(\operatorname{supp} a)$. The last inequality is a direct consequence of (4.21).

Now, we want a bound of the form (4.22) for $B_{\gamma}(\lambda)$. Proceeding as in the proof of Theorem 1.2, we can combine (4.21), (4.24) and (4.26) to get

$$
\left|B_{\gamma}(\lambda)\right| \leq \frac{C \prod_{l=1}^{3} e^{-\frac{1}{2} d\left(0, \gamma_{l} 0\right)}}{\lambda^{j}} \sum_{k=0}^{j} \int_{\gamma(\operatorname{supp} a) \cap\left\{|m|>(2 \lambda)^{-\beta}\right\}} \frac{\lambda^{k \beta}}{|m|^{2 j-k}} \frac{d m}{\left(1-|m|^{2}\right)^{2}}
$$

for some $C>0$. If $d(0, \gamma 0)$ is large enough so that $\gamma(\operatorname{supp} a) \cap B_{0}^{2}\left(\frac{1}{2}\right)=\emptyset$, then $|m|>\frac{1}{2}$ in the integrand and

$$
\begin{aligned}
\left|B_{\gamma}(\lambda)\right| & \leq C^{\prime} j \lambda^{j(\beta-1)} \prod_{l=1}^{3} e^{-\frac{1}{2} d\left(0, \gamma_{l} 0\right)} \int_{\gamma(\operatorname{supp} a)} \frac{d m}{\left(1-|m|^{2}\right)^{2}} \\
& =C^{\prime} j \lambda^{j(\beta-1)} \prod_{l=1}^{3} e^{-\frac{1}{2} d\left(0, \gamma_{l} 0\right)} \operatorname{Vol}_{\mathbb{H}^{2}}(\operatorname{supp} a),
\end{aligned}
$$

for some $C^{\prime}>0$, when $\lambda$ is large. Otherwise, the compact set $\gamma(\operatorname{supp} a)$ is contained in some ball of radius $R<1$ independent of $\gamma$, and (4.27) becomes

$$
\left|B_{\gamma}(\lambda)\right| \leq \frac{C^{\prime \prime} \prod_{l=1}^{3} e^{-\frac{1}{2} d\left(0, \gamma_{l} 0\right)}}{\lambda^{j}} \sum_{k=0}^{j} \int_{B_{0}^{2}(R) \cap\left\{|m|>(2 \lambda)^{-\beta}\right\}} \frac{\lambda^{k \beta}}{|m|^{2 j-k}} d m,
$$


for some $C^{\prime \prime}>0$. Passing to polar coordinates (with $d m=r d \theta d r$ and $r:=|m|$ ) in the last integral, we obtain

$$
\left|B_{\gamma}(\lambda)\right| \leq \frac{\tilde{C} \prod_{l=1}^{3} e^{-\frac{1}{2} d\left(0, \gamma_{l} 0\right)}}{\lambda^{j}} \sum_{k=0}^{j} \int_{(2 \lambda)-\beta}^{R} \frac{\lambda^{k \beta}}{r^{2 j-k-1}} d r \leq \tilde{C}^{\prime} \prod_{l=1}^{3} e^{-\frac{1}{2} d\left(0, \gamma_{l} 0\right)} \lambda^{(2 j-2) \beta-j},
$$

for some $\tilde{C}, \tilde{C}^{\prime}>0$, when $\lambda$ is large enough.

Fix $\beta:=\frac{1}{2}$ and let $j$ be large enough so that $j(\beta-1)>1$. From (4.22), (4.28) and (4.29), we get the upper bound

$$
\left|I_{\gamma}(\lambda)\right| \leq C \prod_{l=1}^{3} e^{-\frac{1}{2} d\left(0, \gamma_{l} 0\right)} \lambda^{-1}
$$

when $\lambda$ is large enough.

Now, consider the case where $\gamma_{1}^{-1} \xi, \gamma_{2}^{-1} \xi$ and $\gamma_{3}^{-1} \xi$ are not all different. If $\gamma_{i}^{-1} \xi=\gamma_{j}^{-1} \xi$ for some $i \neq j$, then at least two of the three unit vectors in the sum $\sum_{k=1}^{3} \tilde{\nabla} \phi_{\gamma_{1}^{-1} \xi}$ are equal, which implies that $\left|\tilde{\nabla}_{m} \psi_{\gamma}(m)\right| \geq 1$. Hence the phase is non stationary and repeated integration by parts gives us

$$
\left|I_{\gamma}(\lambda)\right| \leq C \prod_{l=1}^{3} e^{-\frac{1}{2} d\left(0, \gamma_{l} 0\right)} \lambda^{-k}, \quad \text { for all } k \in \mathbb{N}
$$

uniformly in $\gamma$.

Finally, we apply the argument about the exponent of convergence of the Poincaré series described in $\S 4.1 .3$ to sum over $\Gamma \times \Gamma \times \Gamma$. This provides us with the bound

$$
\sum_{\gamma_{1}, \gamma_{2}, \gamma_{3} \in \Gamma} I_{\gamma}(\lambda)=\mathcal{O}\left(\lambda^{-1}\right)
$$

as $\lambda \rightarrow \infty$. The independence of the implied constant on $\xi \in \partial \bar{X}$ follows from the explanation given at the end of $\S 4$.1.3. This proves the assertion of the proposition when $k=3$. 
Case 2: $k=1$

We use $\xi_{1}=i, \xi_{2}=\sqrt{3} / 2+i / 2$ and $\xi_{3}=-\sqrt{3} / 2+i / 2$. The right-hand side of (4.20) then becomes

$$
\begin{aligned}
\nabla_{m} \psi_{\gamma}\left(\gamma^{-1} m\right) & =\nabla_{m} \phi_{\xi_{1}}(m)-\nabla_{m} \phi_{\xi_{2}}(m)-\nabla_{m} \phi_{\xi_{3}}(m) \\
& =\left(\frac{6\left(2(m-i)-\left(\bar{m}-i \frac{m}{\bar{m}}\right)\right)}{\left(|m|^{2}-1\right)(\bar{m}+i)(\bar{m}-(\sqrt{3} / 2-i / 2))(\bar{m}-(-\sqrt{3} / 2-i / 2))}\right) \bar{m}
\end{aligned}
$$

Since the factor in front of $\bar{m}$ in the previous equation is bounded away from 0 when $m \in \mathbb{D}$, inequality (4.21) holds. An argument along the lines of the one used to treat the previous case then applies directly.

Case 3: $k=0$ or $k=2$

The expression of $\nabla_{m} \psi_{\gamma}\left(\gamma^{-1} m\right)$ differs from the ones of Case 1 and Case 2 respectively only by a minus sign. The same argument applies directly. This concludes the proof of Proposition 4.1. 


\section{CHAPTER 5}

\section{An error term for the fourth moment on surfaces}

In this chapter, we provide a polynomial remainder for the fourth moment of Eisenstein series on surfaces. The bulk of this error term consists in the restricted $L^{4}$-bound given by Theorem 1.3. This is proved in $§ 1.3$. The procedure we will follow is strongly inspired by [16, Lemma 7], which is the main block in the proof of the restricted $L^{2}$-bound given by (1.1). For this reason, the proof of this result will be reproduced in $§ 5.1$. However, as will be seen below, a distinctive feature of the fourth moment considered in this chapter is that we have to deal with stationary phases, as opposed to the equidistribution problem studied by Guillarmou and Naud [16], in which the phases were all non-stationary.

We begin by stating and proving an elementary fact about Möbius transformations that will be useful to prove the two theorems below.

Lemma 5.1. Let $\Gamma$ be a convex co-compact subgroup of $\operatorname{Isom}_{+}\left(\mathbb{H}^{n+1}\right)$. Let $\mathcal{F}$ be a fixed fundamental domain of $\Gamma$. Then there exists a constant $C>0$ such that

$$
\left|\gamma \xi-\gamma^{\prime} \xi\right| \geq C \max \left\{e^{-d(0, \gamma 0)}, e^{-d\left(0, \gamma^{\prime} 0\right)}\right\}
$$

uniformly for all $\xi \in \overline{\mathcal{F}} \cap S^{n}$ and for all $\gamma, \gamma^{\prime} \in \Gamma$ such that $\gamma \neq \gamma^{\prime}$.

Proof. We follow the argument that led to equation (23) in [16]. By Lemma 2.3, with $z=\xi$ and $z^{\prime}=\gamma^{-1} \circ \gamma^{\prime} \xi$, we have

$$
\left|\gamma \xi-\gamma^{\prime} \xi\right|=\frac{\left|\xi-\gamma^{-1} \circ \gamma^{\prime} \xi\right|}{\sinh ^{2}\left(\frac{1}{2} d(0, \gamma 0)\right)\left|\xi-a_{\gamma}\right|\left|\gamma^{-1} \circ \gamma^{\prime} \xi-a_{\gamma}\right|} .
$$

Since $\Gamma$ is discrete and $\xi \in \Omega_{\Gamma}$ is bounded away from the limit set of $\Gamma$, the numerator on the right-hand side of the last equation is bounded from below uniformly in $\gamma \neq \gamma^{\prime}$ and $\xi \in \overline{\mathcal{F}} \cap S^{n}$. Moreover, the discreteness of $\Gamma$, along with Proposition 2.2, implies that $a_{\gamma}$ 
is bounded from above uniformly in $\gamma \in \Gamma$. Therefore, there exists $C>0$ independent of $\gamma \neq \gamma^{\prime}$ and $\xi$ such that

$$
\left|\gamma \xi-\gamma^{\prime} \xi\right| \geq C e^{-d(0, \gamma 0)}
$$

Applying the argument again after interchanging $\gamma$ and $\gamma^{\prime}$, we deduce (5.1).

\subsection{The second moment}

This section is devoted to recalling the proof of (1.1) discussed in the introduction. The intent is to first expose the reader to an argument in the vein of the one used to prove Theorem 1.3, but technically simpler.

The equidistribution result of Guillarmou and Naud can be restated as follows.

Theorem 5.2 (Guillarmou and Naud [16]). If $\delta_{\Gamma}<n / 2$, then for any $a \in C_{0}^{\infty}(X)$,

$$
\int_{X} a(m)\left|E\left(s_{\lambda} ; m, \xi\right)\right|^{2} d v_{X}(m)=\int_{X} a(m) E(n ; m, \xi) d v_{X}(m)+\mathcal{O}\left(\lambda^{2 \delta_{\Gamma}-n}\right),
$$

as $\lambda \rightarrow \infty$, where $d v_{X}(m)$ is the hyperbolic volume measure.

Proof. We follow the argument of the proof of Lemma 7 in [16]. The reader is referred to $\S 3.1$ for the notation that remains unexplained below. By (3.1) and Lemma 3.2, the proof amounts to showing that

$$
\sum_{\gamma \neq \gamma^{\prime}} I_{\gamma, \gamma^{\prime}}(\lambda)=\mathcal{O}\left(\lambda^{2 \delta_{\Gamma}-n}\right)
$$

as $\lambda \rightarrow \infty$, where

$$
I_{\gamma, \gamma^{\prime}}(\lambda):=\int_{X} a(m)\left(E_{0}(1 ; \gamma m, \xi) E_{0}\left(1 ; \gamma^{\prime} m, \xi\right)\right)^{\frac{n}{2}} e^{i \lambda \psi_{\gamma, \gamma^{\prime}}(m)} d v_{X}(m),
$$

and

$$
\psi_{\gamma, \gamma^{\prime}}(m):=\log \left(\frac{E_{0}(1 ; \gamma m, \xi)}{E_{0}\left(1 ; \gamma^{\prime} m, \xi\right)}\right)
$$


A direct calculation (see, e.g., (3.3) and (4.1) above) gives us

$$
\left|\nabla_{m} \psi_{\gamma, \gamma^{\prime}}(m)\right|=\frac{2\left|\gamma^{-1} \xi-\gamma^{\prime-1} \xi\right|}{|m-\gamma \xi|\left|m-\gamma^{\prime} \xi\right|} .
$$

Therefore, the phase function $\psi_{\gamma, \gamma^{\prime}}(m)$ has no stationary points. We can then apply integration by parts to $I_{\gamma, \gamma^{\prime}}$ :

$$
\begin{aligned}
I_{\gamma, \gamma^{\prime}}(\lambda) & =\frac{1}{i \lambda} \int_{X} a(m)\left(E_{0}^{\gamma}(m) E_{0}^{\gamma^{\prime}}(m)\right)^{\frac{n}{2}} \nabla_{m}\left(e^{i \lambda \psi_{\gamma, \gamma^{\prime}}(m)}\right) \cdot \frac{\nabla_{m} \psi_{\gamma, \gamma^{\prime}}(m)}{\left|\nabla_{m} \psi_{\gamma, \gamma^{\prime}}(m)\right|^{2}} d v_{X}(m) \\
& =\frac{1}{i \lambda} \int_{X} e^{i \lambda \psi_{\gamma, \gamma^{\prime}}(m)} \nabla_{m}^{*}\left(a(m)\left(E_{0}^{\gamma}(m) E_{0}^{\gamma^{\prime}}(m)\right)^{\frac{n}{2}} \frac{\nabla_{m} \psi_{\gamma, \gamma^{\prime}}(m)}{\left|\nabla_{m} \psi_{\gamma, \gamma^{\prime}}(m)\right|^{2}}\right) d v_{X}(m) .
\end{aligned}
$$

Now, we want to control the growth of $I_{\gamma, \gamma^{\prime}}(\lambda)$ as $\gamma \neq \gamma^{\prime}$ vary over $\Gamma$. By (4.1) and (5.2), we have

$$
\nabla_{m} \psi_{\gamma, \gamma^{\prime}}(m)=\frac{F_{\gamma^{-1} \xi, \gamma^{-1} \xi}(m)}{G_{\gamma^{-1} \xi, \gamma^{\prime-1} \xi}(m)}
$$

where $F_{\gamma^{-1} \xi, \gamma^{-1} \xi}(m)=0$ if and only if $\gamma=\gamma^{\prime}$, (see also Lemma 3.2) and

$$
\left|G_{\gamma^{-1} \xi, \gamma^{\prime-1} \xi}(m)\right| \geq C, \quad m \in \operatorname{supp} a,
$$

for some constant $C>0$ uniformly in $\gamma \neq \gamma^{\prime}$. Therefore, for all $\alpha \in \mathbb{N}_{0}^{n+1}$, there exists a constant $C_{\alpha}>0$ such that

$$
\left|\partial_{\alpha} \nabla_{m} \psi_{\gamma, \gamma^{\prime}}(m)\right| \leq C_{\alpha}\left|\gamma^{-1} \xi-\gamma^{\prime-1} \xi\right|
$$

for all $m \in \operatorname{supp} a$. Combining (5.2), (5.4) and Lemma 5.1, we deduce the existence of a constant $C_{\alpha}$ independent of $\gamma \neq \gamma^{\prime}$ and $\xi$ such that

$$
\left|\partial_{\alpha} \frac{\nabla_{m} \psi_{\gamma, \gamma^{\prime}}(m)}{\left|\nabla_{m} \psi_{\gamma, \gamma^{\prime}}(m)\right|^{2}}\right| \leq C_{\alpha} \min \left\{e^{d(0, \gamma 0)}, e^{d\left(0, \gamma^{\prime} 0\right)}\right\},
$$

for any $m \in \operatorname{supp} a$. This equation can then be used with Lemma 2.19 to bound the integrand in (5.3). This gives us the inequality

$$
\left|I_{\gamma, \gamma^{\prime}}(\lambda)\right| \leq C \lambda^{-1} e^{-\frac{n}{2}\left(d(0, \gamma 0)+d\left(0, \gamma^{\prime} 0\right)\right)} \min \left\{e^{d(0, \gamma 0)}, e^{d\left(0, \gamma^{\prime} 0\right)}\right\}
$$


for some $C>0$ independent of $\gamma, \gamma^{\prime}$ and $\xi$. If, instead, we perform the integration by parts $j$ times and apply again the argument above, we get

$$
\left|I_{\gamma, \gamma^{\prime}}(\lambda)\right| \leq C \lambda^{-j} e^{-\frac{n}{2}\left(d(0, \gamma 0)+d\left(0, \gamma^{\prime} 0\right)\right)} \min \left\{e^{j d(0, \gamma 0)}, e^{j d\left(0, \gamma^{\prime} 0\right)}\right\} .
$$

It remains to sum up the $I_{\gamma, \gamma^{\prime}}(\lambda)$ over $\Gamma \times \Gamma \backslash$ diag. This is done as follows, by decomposing $\Gamma$ into two parts $\Gamma=\Gamma_{\leq} \sqcup \Gamma_{>}$, where

$$
\Gamma_{\leq}:=\{\gamma \in \Gamma: d(0, \gamma 0) \leq \log (\lambda)\} \text { and } \Gamma_{>}:=\{\gamma \in \Gamma: d(0, \gamma 0)>\log (\lambda)\}
$$

By Theorem 2.16, if $j \geq \frac{n}{2}$, then for any large $\lambda$,

$$
\sum_{d(0, \gamma 0) \leq \log \lambda} e^{\left(j-\frac{n}{2}\right) d(0, \gamma 0)} \leq N(\log \lambda) e^{\left(j-\frac{n}{2}\right) \log \lambda}=\mathcal{O}\left(\lambda^{j-\frac{n}{2}+\delta_{\Gamma}}\right) .
$$

On the other hand, applying Stieltjes integration and using Theorem 2.16 again, we find

$$
\sum_{d(0, \gamma 0)>\log \lambda} e^{-\frac{n}{2} d(0, \gamma 0)} \leq \int_{\log \lambda}^{\infty} e^{-\frac{n}{2} u} d N(u)=\mathcal{O}\left(\lambda^{\delta_{\Gamma}-\frac{n}{2}}\right) .
$$

The proof would be complete if we showed that

$$
\sum_{\substack{\gamma \in \Gamma_{\alpha}, \gamma^{\prime} \in \Gamma_{\beta} \\ \gamma \neq \gamma^{\prime}}} I_{\gamma, \gamma^{\prime}}(\lambda)=\mathcal{O}\left(\lambda^{2 \delta_{\Gamma}-n}\right),
$$

for all $\alpha, \beta \in\{\alpha, \beta\}$. When $\alpha=\beta=>$, the bound $\mathcal{O}\left(\lambda^{2 \delta_{\Gamma}-n}\right)$ is obtained without integration by parts. Indeed, the bound (5.5) with $j=0$ can by found directly from $I_{\gamma, \gamma^{\prime}}(\lambda)$ and the estimates above. One can then apply (5.7) to the sums over both $\gamma$ and $\gamma^{\prime}$.

In the case where $\alpha=\beta=\leq$, we apply integration by parts $2 j$ times, where $j \geq \frac{n}{2}$, and we use (5.6) to conclude that (5.8) holds. In the two remaining cases, we integrate by parts $j$ times, where $j \geq \frac{n}{2}$. The bound $\mathcal{O}\left(\lambda^{2 \delta_{\Gamma}-n}\right)$ is then the result of a combination of both (5.6) and (5.7). This concludes the proof. 
In order to obtain a remainder for the second moment of the real and imaginary parts of the Eisenstein series, i.e., the content of (1.8), the "cross-terms" have to be considered as well. This issue will be discussed at the end of $\S 5.3$ :

\subsection{Proof of Theorem 1.3}

Like with the other results presented so far, the proof of this theorem consists mainly in using the representation of $E\left(s_{\lambda} ; m, \xi\right)$ as an absolutely convergent series over the elements of the group $\Gamma$ in order to study, by means of elementary methods originating from the theory of oscillatory integrals, the limit as $\lambda \rightarrow \infty$ of each of the terms of the resulting series. Unless stated explicitly, the notation used in this section is the one introduced in Chapter 3 and Chapter 4 .

As explained in $\S 3.1$, we lift everything to some fixed fundamental domain $\mathcal{F}$ for $\Gamma$. By Lemma 2.20 and Remark 2.8 we can then write

$$
\left|E\left(s_{\lambda} ; \pi_{\Gamma}(m), \pi_{\Gamma}(\xi)\right)\right|^{2}=E\left(1 ; \pi_{\Gamma}(m), \pi_{\Gamma}(\xi)\right)+\eta(\xi) \sum_{\gamma \neq \gamma^{\prime}}\left(E_{0}^{\gamma}(m) E_{0}^{\gamma^{\prime}}(m)\right)^{\frac{1}{2}}\left(\frac{E_{0}^{\gamma}(m)}{E_{0}^{\gamma^{\prime}}(m)}\right)^{i \lambda},
$$

where $m \in \mathcal{F}$. Therefore,

$$
\begin{aligned}
\left|E\left(s_{\lambda} ; \pi_{\Gamma}(m), \pi_{\Gamma}(\xi)\right)\right|^{4} & =\left(E\left(1 ; \pi_{\Gamma}(m), \pi_{\Gamma}(\xi)\right)\right)^{2} \\
& +2 \eta(\xi) \sum_{\gamma \neq \gamma^{\prime}} E\left(1 ; \pi_{\Gamma}(m), \pi_{\Gamma}(\xi)\right)\left(E_{0}^{\gamma}(m) E_{0}^{\gamma^{\prime}}(m)\right)^{\frac{1}{2}}\left(\frac{E_{0}^{\gamma}(m)}{E_{0}^{\gamma^{\prime}}(m)}\right)^{i \lambda} \\
& +\eta(\xi)^{2} \sum_{\gamma_{1} \neq \gamma_{1}^{\prime}} \sum_{\gamma_{2} \neq \gamma_{2}^{\prime}} \prod_{l=1}^{2}\left(E_{0}^{\gamma_{l}}(m) E_{0}^{\gamma_{l}^{\prime}}(m)\right)^{\frac{1}{2}}\left(\frac{E_{0}^{\gamma_{l}}(m)}{E_{0}^{\gamma_{l}^{\prime}}(m)}\right)^{i \lambda}
\end{aligned}
$$

Using the absolute convergence of the series above, we can consider the integral

$$
\int_{X} a(m)\left|E\left(s_{\lambda} ; m, \xi\right)\right|^{4} d v_{X}(m)
$$

as the sum of the integrals against the test function $a(m)$ of every term on the right-hand side of (5.9). It is easy to see that the second summation in (5.9) gives rise to an integral bounded by $\mathcal{O}\left(\lambda^{2 \delta_{\Gamma}-1}\right)$ as $\lambda \rightarrow \infty$. Indeed, if we define $b_{\xi}(m):=a(m) E\left(n ; \pi_{\Gamma}(m), \pi_{\Gamma}(\xi)\right) \in C_{0}^{\infty}(X)$, 
the proof of Theorem 5.2 can be applied directly to

$$
I_{\gamma, \gamma^{\prime}}(\lambda):=\int_{X} b_{\xi}(m)\left(E_{0}^{\gamma}(m) E_{0}^{\gamma^{\prime}}(m)\right)^{\frac{1}{2}}\left(\frac{E_{0}^{\gamma}(m)}{E_{0}^{\gamma^{\prime}}(m)}\right)^{i \lambda} d v(m),
$$

with $a$ replaced by $b_{\xi}$. (This may be done since supp $b_{\xi} \subset \operatorname{supp} a$ and $b_{\xi}$ is uniformly bounded with respect to $\xi$.) Hence $\sum_{\gamma \neq \gamma^{\prime}} I_{\gamma, \gamma^{\prime}}(\lambda)=\mathcal{O}\left(\lambda^{2 \delta_{\Gamma}-1}\right)$.

As regards the last summation, if $\gamma_{1}^{\prime}=\gamma_{2}$ and $\gamma_{1}=\gamma_{2}^{\prime}$, then the oscillating term is equal to 1 . Summing up the corresponding terms, we obtain

$$
\begin{aligned}
\eta(\xi)^{2} \sum_{\gamma \neq \gamma^{\prime}} E_{0}^{\gamma}(m) E_{0}^{\gamma^{\prime}}(m) & =\left(\sum_{\gamma \in \Gamma} \eta(\xi) E_{0}^{\gamma}(m)\right)^{2}-\sum_{\gamma \in \Gamma}\left(\eta(\xi) E_{0}^{\gamma}(m)\right)^{2}=\left(E\left(1 ; \pi_{\Gamma}(m), \pi_{\Gamma}(\xi)\right)\right)^{2} \\
& -E\left(2 ; \pi_{\Gamma}(m), \pi_{\Gamma}(\xi)\right) .
\end{aligned}
$$

Therefore, in order to establish the theorem, it remains only to show that $\sum I_{\gamma_{1}, \gamma_{1}^{\prime}, \gamma_{2}, \gamma_{2}^{\prime}}(\lambda)=$ $\mathcal{O}\left(\lambda^{\delta_{\Gamma}-\frac{1}{2}}\right)$ where

$$
I_{\gamma_{1}, \gamma_{1}^{\prime}, \gamma_{2}, \gamma_{2}^{\prime}}(\lambda):=\int_{X} a(m) \prod_{l=1}^{2}\left(E_{0}^{\gamma_{l}}(m) E_{0}^{\gamma_{l}^{\prime}}(m)\right)^{\frac{1}{2}}\left(\frac{E_{0}^{\gamma_{l}}(m)}{E_{0}^{\gamma_{l}^{\prime}}(m)}\right)^{i \lambda} d v(m)
$$

and the summation is over $\gamma_{1}, \gamma_{2}, \gamma_{1}^{\prime}, \gamma_{2}^{\prime} \in \Gamma$ for which $\gamma_{1} \neq \gamma_{1}^{\prime}$ and $\gamma_{2} \neq \gamma_{2}^{\prime}$, and either $\gamma_{1}^{\prime} \neq \gamma_{2}$ or $\gamma_{2}^{\prime} \neq \gamma_{1}$. We group these elements into three categories and treat them separately.

\subsubsection{Case 1: $\gamma_{1}^{\prime}=\gamma_{2}$ or $\gamma_{2}^{\prime}=\gamma_{1}$.}

Without loss of generality, suppose that $\gamma:=\gamma_{1}^{\prime}=\gamma_{2}$. For any $\gamma_{a}, \gamma_{b} \in\left\{\gamma, \gamma_{1}, \gamma_{2}^{\prime}\right\}$, define

$$
\begin{aligned}
& I_{\gamma_{a}, \gamma_{b}}(\lambda):=\int_{X} b_{\xi}(m)\left(E_{0}^{\gamma_{a}}(m) E_{0}^{\gamma_{b}}(m)\right)^{\frac{1}{2}}\left(\frac{E_{0}^{\gamma_{1}}(m)}{E_{0}^{\gamma_{2}^{\prime}}(m)}\right)^{i \lambda} d v(m), \\
& I_{\gamma_{a}, \gamma_{b}}^{\prime}(\lambda):=\int_{X} a(m)\left(\left(E_{0}^{\gamma_{a}}(m)\right)^{3} E_{0}^{\gamma_{b}}(m)\right)^{\frac{1}{2}}\left(\frac{E_{0}^{\gamma_{1}}(m)}{E_{0}^{\gamma_{2}^{\prime}}(m)}\right)^{i \lambda} d v(m),
\end{aligned}
$$

where $b_{\xi}(m):=a(m) E(2 ; m, \xi) \in C_{0}^{\infty}(X)$. In the same spirit as in $\S 3.1$, we can reorganize the sum of $I_{\gamma_{1}, \gamma, \gamma, \gamma_{2}^{\prime}}$ over $\gamma \neq \gamma_{1} \neq \gamma_{2}^{\prime} \neq \gamma$ as follows:

$$
\sum_{\gamma \neq \gamma_{1} \neq \gamma_{2}^{\prime} \neq \gamma} I_{\gamma_{1}, \gamma, \gamma, \gamma_{2}^{\prime}}(\lambda)=\sum_{\gamma_{1} \neq \gamma_{2}^{\prime}} I_{\gamma_{1}, \gamma_{2}^{\prime}}(\lambda)-\sum_{\gamma \neq \gamma_{2}^{\prime}} I_{\gamma, \gamma_{2}^{\prime}}^{\prime}(\lambda)-\sum_{\gamma_{1} \neq \gamma} I_{\gamma, \gamma_{1}}^{\prime}(\lambda) .
$$


Notice that the last two sums correspond to the cases where $\gamma=\gamma_{1} \neq \gamma_{2}^{\prime}$ and $\gamma=\gamma_{2}^{\prime} \neq \gamma_{1}$ respectively. For $\sum I_{\gamma_{1}, \gamma_{2}^{\prime}}(\lambda)$, the proof of Theorem 5.2 applies directly. Hence $\sum\left|I_{\gamma_{1}, \gamma_{2}^{\prime}}(\lambda)\right|=$ $\mathcal{O}\left(\lambda^{2 \delta_{\Gamma}-1}\right)$. As for $\sum I_{\gamma, \gamma_{2}^{\prime}}^{\prime}(\lambda)$ and $\sum I_{\gamma_{1}, \gamma}^{\prime}(\lambda)$, the proof also applies after noticing (see Lemma 2.19) that $\left|\left(E_{0}^{\gamma}(m)\right)^{\frac{3}{2}}\right| \leq C e^{-\frac{1}{2} d(0, \gamma 0)}$. Therefore, the right-hand side of $(5.10)$ is $\mathcal{O}\left(\lambda^{2 \delta_{\Gamma}-1}\right)$ as $\lambda \rightarrow \infty$.

Before treating the remaining cases, we introduce a notation that will simplify the equations. Define $\xi_{i}:=\gamma_{i}^{-1} \xi, \xi_{i}^{\prime}:=\gamma_{i}^{\prime-1} \xi$ and $\gamma:=\left(\xi_{1}, \xi_{1}^{\prime}, \xi_{2}, \xi_{2}^{\prime}\right) \in \mathbb{S}^{4}$. Moreover, let $f_{\gamma}(m)$ be the unique function satisfying

$$
\left(\frac{E_{0}^{\gamma_{1}}(m)}{E_{0}^{\gamma_{1}^{\prime}}(m)} \frac{E_{0}^{\gamma_{2}}(m)}{E_{0}^{\gamma_{2}^{\prime}}(m)}\right)^{i \lambda}=e^{i f_{\gamma}(m) \lambda}
$$

If we write $\psi_{\gamma}:=\phi_{\xi_{1}}-\phi_{\xi_{1}^{\prime}}+\phi_{\xi_{2}}-\phi_{\xi_{2}^{\prime}}$, where $\phi_{\xi}$ is the usual Busemann function defined in $\S 2.2 .4$, then Lemma 2.18 gives us

$$
f_{\gamma}(m)=\psi_{\gamma}(m)-\log \left|D \gamma_{1}\left(\xi_{1}\right)\right|+\log \left|D \gamma_{1}^{\prime}\left(\xi_{1}^{\prime}\right)\right|-\log \left|D \gamma_{2}\left(\xi_{2}\right)\right|+\log \left|D \gamma_{2}^{\prime}\left(\xi_{2}^{\prime}\right)\right|
$$

We now use the explicit expression of $\nabla \phi_{\xi}$ calculated in $\S 3.1$ (see (3.3)) to write the gradient of $f_{\gamma}$ as a function of $m, \bar{m}$ and $\gamma$ :

$$
\begin{aligned}
\nabla_{m} f_{\gamma}(m) & =\nabla_{m} \psi_{\gamma}(m)=2\left(\frac{m-\xi_{1}^{\prime}}{\left|m-\xi_{1}^{\prime}\right|^{2}}-\frac{m-\xi_{1}}{\left|m-\xi_{1}\right|^{2}}+\frac{m-\xi_{2}^{\prime}}{\left|m-\xi_{2}^{\prime}\right|^{2}}-\frac{m-\xi_{2}}{\left|m-\xi_{2}\right|^{2}}\right) \\
& =\frac{2 F_{\bar{\gamma}}(\bar{m})}{G_{\bar{\gamma}}(\bar{m})}
\end{aligned}
$$

where $G_{\gamma}(m):=\left(m-\xi_{1}^{\prime}\right)\left(m-\xi_{1}\right)\left(m-\xi_{2}^{\prime}\right)\left(m-\xi_{2}\right)$ and

$$
F_{\gamma}(m):=m^{2}\left(\xi_{2}^{\prime}-\xi_{2}+\xi_{1}^{\prime}-\xi_{1}\right)-2 m\left(\xi_{2}^{\prime} \xi_{1}^{\prime}-\xi_{2} \xi_{1}\right)+\xi_{1}^{\prime} \xi_{1} \xi_{2}^{\prime} \xi_{2}\left(\overline{\xi_{2}}-\overline{\xi_{2}^{\prime}}+\overline{\xi_{1}}-\overline{\xi_{1}^{\prime}}\right)
$$

The proof of the remaining cases consists once again in studying the stationary points (if any) of the phase functions $f_{\gamma}$ according to the different possible configurations $\gamma \in \mathbb{S}^{4}$. Like in the proof of Proposition 4.1, we will make an essential use of isometries of $\mathbb{H}^{2}$ in order to reduce this task to solving an elementary problem of Euclidean geometry in the 
plane. A considerable part of the proof will then be devoted to controlling the effect of these isometries using the tools developed in $\S 4.2$.

\subsubsection{Case 2: The elements $\gamma_{1}, \gamma_{2}, \gamma_{1}^{\prime}$ and $\gamma_{2}^{\prime}$ are all different.}

Since $G_{\bar{\gamma}}(\bar{m})$ is bounded in any compact subset of $\mathbb{D}$ and $F_{\bar{\gamma}}(\bar{m})=\overline{F_{\gamma}(m)}$, finding the stationary points of $\psi_{\gamma}(m)$ boils down to finding the zero set of $F_{\gamma}(m)$.

The function $F_{\gamma}(m)$ is a complex polynomial of degree 2 , which means that $\psi_{\gamma}(m)$ has at most two stationary points in $\mathbb{D}$. We claim that there is actually at most one, and that it corresponds to the intersection (if it exists) of the geodesic joining $\xi_{1}$ to $\xi_{2}$ and the one joining $\xi_{1}^{\prime}$ to $\xi_{2}^{\prime}$. Indeed, it is an elementary geometry fact that four unit Euclidean vectors in the plane cancel if and only if to each vector corresponds a vector pointing in the opposite direction. In other words, they must either be arranged in an $X$-shape or they must form a line. Notice, however, that this characteristic fails in higher dimension. It follows that the sum of unit vectors

$$
\tilde{\nabla}_{m} \psi_{\gamma}(m)=\tilde{\nabla}_{m} \phi_{\xi_{1}}(m)-\tilde{\nabla}_{m} \phi_{\xi_{1}^{\prime}}(m)+\tilde{\nabla}_{m} \phi_{\xi_{2}}(m)-\tilde{\nabla}_{m} \phi_{\xi_{2}^{\prime}}(m)
$$

vanishes if and only if either $\xi_{i}=\xi_{i}^{\prime}$ for $i=1,2$, or

$$
\tilde{\nabla}_{m} \phi_{\xi_{1}}(m)=-\tilde{\nabla}_{m} \phi_{\xi_{2}}(m) \text { and } \tilde{\nabla}_{m} \phi_{\xi_{1}^{\prime}}(m)=-\tilde{\nabla}_{m} \phi_{\xi_{2}^{\prime}}(m)
$$

The first possibility occurs only if at least two of $\gamma_{1}, \gamma_{2}, \gamma_{1}^{\prime}$ and $\gamma_{2}^{\prime}$ are equal, which never happens in the case under consideration. Since $\tilde{\nabla}_{m} \phi_{\xi}(m)$ corresponds to the unit vector pointing in the direction of the geodesic linking $m$ to $\xi$, the second possibility implies that $\xi_{2}$ is the point on the unit circle opposite to $\xi_{1}$ along the geodesic linking $m$ to $\xi_{1}$; similarly for $\xi_{2}^{\prime}$ and $\xi_{1}^{\prime}$. In other words, $m$ must lie on the geodesic joining $\xi_{1}$ to $\xi_{2}$ as well as on the one joining $\xi_{1}^{\prime}$ to $\xi_{2}^{\prime}$. Therefore, $m$ must be the intersection of those two geodesics, which was the claim.

According to this claim, it makes sense to subdivide Case 2 further, depending on whether the configuration $\left(\xi_{1}, \xi_{1}^{\prime}, \xi_{2}, \xi_{2}^{\prime}\right) \in \mathbb{S}^{4}$ gives rise to a stationary point or not. 


\section{Case 2.1: The geodesic between $\xi_{1}$ and $\xi_{2}$, and the one between $\xi_{1}^{\prime}$ and $\xi_{2}^{\prime}$ intersect.}

Let $m_{0} \in \mathbb{D}$ be the intersection point of both geodesics, the one joining $\xi_{1}$ and $\xi_{2}$, and the one joining $\xi_{1}^{\prime}$ and $\xi_{2}^{\prime}$. Let $\gamma_{0} \in \mathrm{PSL}_{2}(\mathbb{R})$ be an isometry sending the point $m_{0}$ to the origin. Applying the same argument as the one used to prove (4.19), we get

$$
\nabla_{m} \psi_{\gamma}\left(\gamma_{0}^{-1} m\right)=\nabla_{m} \phi_{\gamma_{0} \xi_{1}}(m)-\nabla_{m} \phi_{\gamma_{0} \xi_{1}^{\prime}}(m)+\nabla_{m} \phi_{\gamma_{0} \xi_{2}}(m)-\nabla_{m} \phi_{\gamma_{0} \xi_{2}^{\prime}}(m)
$$

In view of the claim above, the only solution of $\nabla_{m} \psi_{\gamma}\left(\gamma_{0}^{-1} m\right)=0$ is $m=0$. It follows from (5.11), (5.12) and the right-hand side of (5.13), that 0 is a root of the polynomial $F_{\gamma_{0} \gamma}(m)$, where $\gamma_{0} \gamma$ is a notation for $\left(\gamma_{0} \xi_{1}, \gamma_{0} \xi_{1}^{\prime}, \gamma_{0} \xi_{2}, \gamma_{0} \xi_{2}^{\prime}\right) \in \mathbb{S}^{4}$. By (5.12), this happens if and only if

$$
\overline{\gamma_{0} \xi_{2}}-\overline{\gamma_{0} \xi_{2}^{\prime}}+\overline{\gamma_{0} \xi_{1}}-\overline{\gamma_{0} \xi_{1}^{\prime}}=0
$$

in which case $F_{\gamma_{0} \gamma}(m)$ takes the simpler form $-2 m\left(\gamma_{0} \xi_{2}^{\prime} \gamma_{0} \xi_{1}^{\prime}-\gamma_{0} \xi_{2} \gamma_{0} \xi_{1}\right)$. From (5.14) and the fact that the points $\xi_{1}, \xi_{1}^{\prime}, \xi_{2}$ and $\xi_{2}^{\prime}$ are distinct points on the unit circles, we deduce that $\gamma_{0} \xi_{2}=-\gamma_{0} \xi_{1}$ and $\gamma_{0} \xi_{2}^{\prime}=-\gamma_{0} \xi_{1}^{\prime}$. Hence $F_{\gamma_{0} \gamma}$ and $G_{\gamma_{0} \gamma}(m)$ simplify further to

$$
\begin{aligned}
& F_{\gamma_{0} \gamma}(m)=-2 m\left(\gamma_{0} \xi_{1}-\gamma_{0} \xi_{1}^{\prime}\right)\left(\gamma_{0} \xi_{1}+\gamma_{0} \xi_{1}^{\prime}\right), \\
& G_{\gamma_{0} \gamma}(m)=\left(m-\gamma_{0} \xi_{1}^{\prime}\right)\left(m-\gamma_{0} \xi_{1}\right)\left(m+\gamma_{0} \xi_{1}^{\prime}\right)\left(m+\gamma_{0} \xi_{1}\right) .
\end{aligned}
$$

Since $\gamma_{0} \xi_{1}$ and $\gamma_{0} \xi_{1}^{\prime}$ are points lying on the unit circle, it follows from elementary Euclidean geometry that the vectors $\gamma_{0} \xi_{1}-\gamma_{0} \xi_{1}^{\prime}$ and $\gamma_{0} \xi_{1}+\gamma_{0} \xi_{1}^{\prime}$ are at least of length $\sqrt{2}$. Hence

$$
\begin{aligned}
\left|\nabla_{m} \psi_{\gamma}\left(\gamma_{0}^{-1} m\right)\right| & =\frac{2\left|F_{\gamma_{0} \gamma}(m)\right|}{\left|G_{\gamma_{0} \gamma}(m)\right|} \\
& \geq \frac{4 \sqrt{2}|m| \min \left\{\left|\gamma_{0} \xi_{1}-\gamma_{0} \xi_{1}^{\prime}\right|\left|\gamma_{0} \xi_{1}+\gamma_{0} \xi_{1}^{\prime}\right|\right\}}{\left|m-\gamma_{0} \xi_{1}^{\prime}\right|\left|m-\gamma_{0} \xi_{1}\right|\left|m+\gamma_{0} \xi_{1}^{\prime}\right|\left|m+\gamma_{0} \xi_{1}\right|}
\end{aligned}
$$

Suppose first that $\gamma_{0} \xi_{1}$ is closer to $\gamma_{0} \xi_{1}^{\prime}$ than to $-\gamma_{0} \xi_{1}^{\prime}$. In this case,

$$
\left|\nabla_{m} \psi_{\gamma}\left(\gamma_{0}^{-1} m\right)\right| \geq \frac{C|m|\left|\gamma_{0} \xi_{1}-\gamma_{0} \xi_{1}^{\prime}\right|}{\left|m-\gamma_{0} \xi_{1}\right|\left|m-\gamma_{0} \xi_{1}^{\prime}\right|},
$$


for some numerical constant $C>0$. Note that we have used the trivial fact that $\left|m+\gamma_{0} \xi_{1}^{\prime}\right| \leq 2$ and $\left|m+\gamma_{0} \xi_{1}\right| \leq 2$. By Proposition 2.3, we have

$$
\left|\nabla_{m} \psi_{\gamma_{0} \gamma}\left(\gamma_{0}^{-1} m\right)\right| \geq \frac{C|m|\left|\xi_{1}-\xi_{1}^{\prime}\right| \sinh ^{2}\left(\frac{1}{2} d\left(0, \gamma_{0} 0\right)\right)\left|\gamma_{0}^{-1} m-a_{\gamma_{0}}\right|^{2}}{\left|\gamma_{0}^{-1} m-\xi_{1}\right|\left|\gamma_{0}^{-1} m-\xi_{1}^{\prime}\right|} .
$$

Applying Lemma 2.4 to $\sinh ^{2}\left(\frac{1}{2} d\left(0, \gamma_{0} 0\right)\right)$ with $m$ replaced by $\gamma_{0}^{-1} m$, we obtain

$$
\left|\nabla_{m} \psi_{\gamma}\left(\gamma_{0}^{-1} m\right)\right|=\frac{C|m|\left|\xi_{1}-\xi_{1}^{\prime}\right|\left(1-\left|\gamma_{0}^{-1} m\right|^{2}\right)}{\left(1-|m|^{2}\right)\left|\gamma_{0}^{-1} m-\xi_{1}\right|\left|\gamma_{0}^{-1} m-\xi_{1}^{\prime}\right|}
$$

Therefore, for $m \in \gamma_{0}(\operatorname{supp} a)$, we have

$$
\left|\nabla_{m} \psi_{\gamma}\left(\gamma_{0}^{-1} m\right)\right| \geq \frac{C|m|\left|\xi_{i}-\xi_{i}^{\prime}\right|}{\left(1-|m|^{2}\right)}
$$

where $i=1$ and $C$ is some positive constant depending only on supp $a$. Notice that the same argument can be applied if we replace $\gamma_{1}$ by $\gamma_{2}$ and $\gamma_{1}^{\prime}$ by $\gamma_{2}^{\prime}$. This leads us to conclude that (5.15) is also valid for $i=2$. Hence

$$
\left|\nabla_{m} \psi_{\gamma}\left(\gamma_{0}^{-1} m\right)\right| \geq \frac{C|m| \max \left\{\left|\xi_{1}-\xi_{1}^{\prime}\right|,\left|\xi_{2}-\xi_{2}^{\prime}\right|\right\}}{\left(1-|m|^{2}\right)}
$$

On the other hand, differentiating (5.12) allows us to deduce that

$$
\left|\partial_{m}^{\alpha}\left(\partial_{j} \psi_{\gamma}(m)\right)\right| \leq C_{\alpha} \max \left\{\left|\xi_{1}-\xi_{1}^{\prime}\right|,\left|\xi_{2}-\xi_{2}^{\prime}\right|\right\}
$$

for $m$ ranging over a compact subset of $\mathbb{D}$. We can then use Lemma 2.5 (with $F(m):=$ $\partial_{j} \psi_{\gamma}(m)$ and $\left.K:=\operatorname{supp} a\right)$ along with an argument similar to the one that led to (4.26) to deduce from (5.16) and the last inequality that

$$
\left|\partial_{\alpha} \frac{\nabla_{m} \psi_{\gamma}\left(\gamma_{0}^{-1} m\right)}{\left|\nabla_{m} \psi_{\gamma}\left(\gamma_{0}^{-1} m\right)\right|^{2}}\right| \leq \frac{C_{\alpha}}{|m|^{|\alpha|+1}\left(1-|m|^{2}\right)^{|\alpha|-1} \max \left\{\left|\xi_{1}-\xi_{1}^{\prime}\right|,\left|\xi_{2}-\xi_{2}^{\prime}\right|\right\}}
$$

By Lemma 5.1, we can bound the right-hand side of (5.17) further to obtain an expression which will be more tractable when we will sum over the elements of the group:

$$
\left|\partial_{\alpha} \frac{\nabla_{m} \psi_{\gamma}\left(\gamma_{0}^{-1} m\right)}{\left|\nabla_{m} \psi_{\gamma}\left(\gamma_{0}^{-1} m\right)\right|^{2}}\right| \leq \frac{C_{\alpha} \min \left\{e^{d\left(0, \gamma_{1} 0\right)}, e^{d\left(0, \gamma_{1}^{\prime} 0\right)}, e^{d\left(0, \gamma_{2} 0\right)}, e^{d\left(0, \gamma_{2}^{\prime} 0\right)}\right\}}{|m|^{|\alpha|+1}\left(1-|m|^{2}\right)^{|\alpha|-1}},
$$


for $C_{\alpha}>0$ depending only on $\alpha \in \mathbb{N}^{2}$ and on supp $a$.

We can now remove the hypothesis that $\gamma_{0} \xi_{1}$ is closer to $\gamma_{0} \xi_{1}^{\prime}$ than to $\gamma_{0} \xi_{2}^{\prime}=-\gamma_{0} \xi_{1}^{\prime}$, by noticing that if it is not the case, the bounds

$$
\left|\nabla_{m} \psi_{\gamma}\left(\gamma_{0}^{-1} m\right)\right| \geq \frac{C|m|\left|\xi_{1}-\xi_{2}^{\prime}\right|}{\left(1-|m|^{2}\right)} \quad \text { and } \quad\left|\nabla_{m} \psi_{\gamma}\left(\gamma_{0}^{-1} m\right)\right| \geq \frac{C|m|\left|\xi_{2}-\xi_{1}^{\prime}\right|}{\left(1-|m|^{2}\right)}
$$

replace (5.15) for $i=1$ and $i=2$ respectively. Essentially the same argument that allowed us to derive (5.18) would give us the same bound again.

Let $I_{\gamma}(\lambda), A_{\gamma}(\lambda), B_{\gamma}(\lambda)$ and $S_{\gamma}$ be as in the proof of Proposition 4.1. Using the Weyl's tube formula [43] in the same way as we derived (4.22), we deduce that when $\lambda$ is large,

$$
\left|A_{\gamma}(\lambda)\right| \leq C \lambda^{-2 \beta} e^{-\frac{1}{2}\left(d\left(0, \gamma_{1} 0\right)+d\left(0, \gamma_{1}^{\prime} 0\right)+d\left(0, \gamma_{2} 0\right)+d\left(0, \gamma_{2}^{\prime} 0\right)\right)}
$$

where $\beta>0$ is the undetermined constant entering in the definition of $A_{\gamma}(\lambda)$. Like in the proof of Proposition 4.1, this constant will be fixed later. Applying integration by parts $j$ times to $B_{\gamma}(\lambda)$ as in the proof of Proposition 4.1, we obtain (4.23) with

$$
u_{\gamma}(m):=\frac{a\left(\gamma^{-1} m\right)}{\left(1-|m|^{2}\right)^{2}} \prod_{l=1}^{2}\left(E_{0}^{\gamma_{l} \gamma^{-1}}(m) E_{0}^{\gamma_{l}^{\prime} \gamma^{-1}}(m)\right)^{\frac{1}{2}}
$$

Combining (5.18), the corresponding analogue of (4.24) and property (iv) of the function $\rho_{\gamma}^{\lambda^{\beta}}(m)($ see $\S 4.1 .2)$, we obtain

$$
\left|B_{\gamma}(\lambda)\right| \leq \frac{C v_{\gamma}^{(j)}(\lambda)}{\lambda^{j}} \sum_{k=1}^{j} \int_{\gamma(\operatorname{supp} a) \cap\left\{|m|>(2 \lambda)^{-\beta}\right\}} \frac{\lambda^{k \beta}}{|m|^{2 j-k}} \frac{d m}{\left(1-|m|^{2}\right)^{2}}
$$

where

$$
v_{\gamma}^{(j)}(\lambda):=\min _{\gamma \in\left\{\gamma_{1}, \gamma_{2}, \gamma_{1}^{\prime}, \gamma_{2}^{\prime}\right\}}\left\{e^{j d(0, \gamma 0)}\right\} e^{-\frac{1}{2}\left(d\left(0, \gamma_{1} 0\right)+d\left(0, \gamma_{1}^{\prime} 0\right)+d\left(0, \gamma_{2} 0\right)+d\left(0, \gamma_{2}^{\prime} 0\right)\right)} .
$$

Like we did in the proof of Proposition 4.1, we consider two cases, depending on the value of $d\left(0, \gamma_{0} 0\right)$. If $d(0, \gamma 0)$ is large enough so that $\gamma(\operatorname{supp} a) \cap B_{0}^{2}\left(\frac{1}{2}\right)=\emptyset$, then we obtain an 
analogue of (4.28), namely

$$
\left|B_{\gamma}(\lambda)\right| \leq C^{\prime} j \lambda^{j(\beta-1)} v_{\gamma}^{(j)}(\lambda) \operatorname{Vol}_{\mathbb{H}^{2}}(\operatorname{supp} a)
$$

when $\lambda$ is large. Otherwise, $\gamma(\operatorname{supp} a)$ is contained in $B_{0}^{2}(R)$ for some $R<1$ independent of $\gamma$, and

$$
\left|B_{\gamma}(\lambda)\right| \leq \frac{C v_{\gamma}^{(j)}(\lambda)}{\lambda^{j}} \sum_{k=1}^{j} \int_{B_{0}^{2}(R) \cap\left\{|m|>(2 \lambda)^{-\beta}\right\}} \frac{\lambda^{k \beta}}{|m|^{2 j-k}} \frac{d m}{\left(1-|m|^{2}\right)^{2}} .
$$

Using polar coordinates, the volume element $d m$ takes the form $d m=r d \theta d r$ and

$$
\left|B_{\gamma}(\lambda)\right| \leq \frac{C^{\prime} v_{\gamma}^{(j)}(\lambda)}{\lambda^{j}} \sum_{k=0}^{j} \int_{(2 \lambda)^{-\beta}}^{R} \frac{\lambda^{k \beta}}{r^{2 j-k-1}} d r \leq C^{\prime \prime} v_{\gamma}^{(j)}(\lambda) \lambda^{(2 j-2) \beta-j}
$$

assuming that $j \geq 3$ and $\lambda$ is large enough. The rate of convergence is optimized by choosing $\beta:=\frac{1}{2}$. From (5.19) and (5.22), we get $\left|I_{\gamma}(\lambda)\right| \leq C v_{\gamma}^{(j)}(\lambda) \lambda^{-1}$ when $\lambda$ is large enough.

We can now sum the $I_{\gamma_{1}, \gamma_{1}^{\prime}, \gamma_{2}, \gamma_{2}^{\prime}}$ 's over all the elements of $\Gamma^{4}$ corresponding to Case 2.1. We decompose $\Gamma$ into $\Gamma=\Gamma_{\leq} \sqcup \Gamma_{>}$where $\Gamma_{\leq}$corresponds to the elements $\gamma \in \Gamma$ such that $d(0, \gamma 0) \leq \alpha \log \lambda$ for some constant $\alpha>0$ that will be fixed later, and $\Gamma_{>}$corresponds to the elements $\gamma \in \Gamma$ such that $d(0, \gamma 0)>\alpha \log \lambda$. Such a decomposition of $\Gamma$ gives rise to sixteen different configurations of $\Gamma_{\alpha_{1}} \times \Gamma_{\alpha_{2}} \times \Gamma_{\alpha_{3}} \times \Gamma_{\alpha_{4}}$ with $\alpha_{i} \in\{\leq,>\}$ and $i=1, \ldots, 4$. We have the trivial inequality

$$
\min _{\gamma \in\left\{\gamma_{1}, \gamma_{2}, \gamma_{1}^{\prime}, \gamma_{2}^{\prime}\right\}}\left\{e^{j d(0, \gamma 0)}\right\} \leq e^{a_{1} d\left(0, \gamma_{1} 0\right)+a_{2} d\left(0, \gamma_{1}^{\prime} 0\right)+a_{3} d\left(0, \gamma_{2} 0\right)+a_{4} d\left(0, \gamma_{2}^{\prime} 0\right)}
$$

for any $a_{1}, a_{2}, a_{3}, a_{4} \geq 0$ such that $a_{1}+a_{2}+a_{3}+a_{4}=j$. By Theorem 2.16, we find an upper bound for the sum over the elements of $\Gamma_{\leq}$:

$$
\sum_{d(0, \gamma 0)>\alpha \log \lambda} e^{-\frac{1}{2} d(0, \gamma 0)} \leq \int_{\alpha \log \lambda}^{\infty} e^{-\frac{1}{2} u} d N(u)=\mathcal{O}\left(\lambda^{\alpha\left(\delta_{\Gamma}-\frac{1}{2}\right)}\right) .
$$


If we assume further that $a_{i} \geq \frac{1}{2}$, then we obtain the following bound for the sum over the elements of $\Gamma_{>}$:

$$
\sum_{d(0, \gamma 0) \leq \alpha \log \lambda} e^{\left(a_{i}-\frac{1}{2}\right) d(0, \gamma 0)}=\mathcal{O}\left(\lambda^{\alpha\left(a_{i}+\delta_{\Gamma}-\frac{1}{2}\right)}\right), \quad a_{i} \geq \frac{1}{2}
$$

We can then combine $(5.23),(5.24)$ and (5.25) to derive a bound for the sum of $I_{\gamma_{1}, \gamma_{1}^{\prime}, \gamma_{2}, \gamma_{2}^{\prime}}$. Namely,

$$
\sum I_{\gamma_{1}, \gamma_{1}^{\prime}, \gamma_{2}, \gamma_{2}^{\prime}}(\lambda)= \begin{cases}\mathcal{O}\left(\lambda^{4 \alpha\left(\delta_{\Gamma}-\frac{1}{2}\right)}\right), & \text { if } \alpha_{i}=>, \text { for all } i \\ \mathcal{O}\left(\lambda^{\alpha\left(4 \delta_{\Gamma}+j-2\right)-1}\right), & \text { if } \alpha_{i}=\leq, \text { for at least one } i\end{cases}
$$

where the sum is over the subset of $\Gamma_{\alpha_{1}} \times \Gamma_{\alpha_{2}} \times \Gamma_{\alpha_{3}} \times \Gamma_{\alpha_{4}}$ with $\alpha_{i} \in\{\leq,>\}$ and $i=1, \ldots, 4$ containing all quadruplets of distinct elements $\left(\gamma_{1}, \gamma_{1}^{\prime}, \gamma_{2}, \gamma_{2}^{\prime}\right)$ giving rise to a stationary point $m_{0}=\gamma_{0}^{-1} 0$.

It remains only to fix the positive constant $\alpha$ so that the bound that we found be in agreement with the one in the statement of the theorem. For $\alpha=\frac{1}{j}$, the sum above becomes $\mathcal{O}\left(\lambda^{\frac{4}{j}\left(\delta_{\Gamma}-\frac{1}{2}\right)}\right)$. Finally, we fix $j=4$, so that we obtain

$$
\sum I_{\gamma_{1}, \gamma_{1}^{\prime}, \gamma_{2}, \gamma_{2}^{\prime}}(\lambda)=\mathcal{O}\left(\lambda^{\delta_{\Gamma}-\frac{1}{2}}\right)
$$

as $\lambda \rightarrow \infty$.

\section{Case 2.2: The geodesic between $\xi_{1}$ and $\xi_{2}$, and the one between $\xi_{1}^{\prime}$ and $\xi_{2}^{\prime}$ do not intersect.}

If the geodesic joining $\xi_{1}$ and $\xi_{2}$, and the one joining $\xi_{1}^{\prime}$ and $\xi_{2}^{\prime}$ are disjoint, then necessarily, for some choice of $(j, k) \in\{(1,2),(2,1)\}$, the geodesic between $\xi_{1}$ and $\xi_{j}^{\prime}$, and the one between $\xi_{2}$ and $\xi_{k}^{\prime}$ will intersect at some point $m_{0} \in \mathbb{D}$. Without loss of generality, assume that $(j, k)=(2,1)$. As before, let $\gamma_{0} \in \mathrm{PSL}_{2}(\mathbb{R})$ be an isometry sending the point $m_{0}$ to the origin. The point $0=\gamma_{0} m_{0}$ is then a solution of the equation $F_{\gamma_{0}}(m)=0$ (see (5.12) above), where $\tilde{\gamma}$ corresponds to $\gamma$ in which $\xi_{2}$ has been interchanged with $\xi_{2}^{\prime}$, i.e., $\tilde{\gamma}:=\left(\xi_{1}, \xi_{1}^{\prime}, \xi_{2}^{\prime}, \xi_{2}\right)$. Modifying (5.14) accordingly, it follows that $\gamma_{0} \xi_{2}-\gamma_{0} \xi_{2}^{\prime}=\gamma_{0} \xi_{1}-\gamma_{0} \xi_{1}^{\prime}$. We can then deduce from the fact that $\xi_{i}$ and $\xi_{j}^{\prime}$ are distinct points on the unit circle that $\gamma_{0} \xi_{2}^{\prime}=-\gamma_{0} \xi_{1}$ and 
$\gamma_{0} \xi_{2}=-\gamma \xi_{1}^{\prime}$. Therefore,

$$
\left|\nabla_{m} \psi_{\gamma}\left(\gamma_{0}^{-1} m\right)\right|=\frac{4\left|m^{2}+\left(\gamma_{0} \xi_{1}\right)\left(\gamma_{0} \xi_{1}^{\prime}\right)\right|\left|\gamma_{0} \xi_{1}-\gamma_{0} \xi_{1}^{\prime}\right|}{\left|m-\gamma_{0} \xi_{1}\right|\left|m+\gamma_{0} \xi_{1}\right|\left|m-\gamma_{0} \xi_{1}^{\prime}\right|\left|m+\gamma_{0} \xi_{1}^{\prime}\right|} .
$$

By (2.5) of Proposition 2.3, for all $m \in \gamma_{0}(\operatorname{supp} a)$, we have

$$
\lim _{d\left(0, \gamma_{0} 0\right) \rightarrow \infty}\left(\gamma_{0}\left(\gamma_{0}^{-1} m\right)-\gamma_{0} \xi_{1}\right)\left(\gamma_{0}\left(\gamma_{0}^{-1} m\right)-\gamma_{0} \xi_{1}^{\prime}\right)=0
$$

Rewriting $m^{2}+\left(\gamma_{0} \xi_{1}\right)\left(\gamma_{0} \xi_{1}^{\prime}\right)$ as $\left(m-\gamma_{0} \xi_{1}\right)\left(m-\gamma_{0} \xi_{1}^{\prime}\right)+m\left(\gamma_{0} \xi_{1}+\gamma_{0} \xi_{1}^{\prime}\right)$ and using the last equation, we get for any $m \in \gamma_{0}(\operatorname{supp} a)$ that

$$
\lim _{d\left(0, \gamma_{0} 0\right) \rightarrow \infty}\left|m^{2}+\left(\gamma_{0} \xi_{1}\right)\left(\gamma_{0} \xi_{1}^{\prime}\right)\right|=\left|\gamma_{0} \xi_{1}+\gamma_{0} \xi_{1}^{\prime}\right|
$$

Moreover, it is easy to see that this quantity does not vanish. Indeed, this would contradict the fact that $\left|\gamma_{0} \xi_{1}-\gamma_{0} \xi_{1}^{\prime}\right| \rightarrow 0$ as $d\left(0, \gamma_{0} 0\right) \rightarrow \infty$, which is a direct consequence of (2.5). We can then adapt the argument invoked in Case 2.1 when deriving (5.16). This gives us the estimate

$$
\left|\nabla_{m} \psi_{\gamma}\left(\gamma_{0}^{-1} m\right)\right| \geq C \frac{\max \left\{\left|\xi_{1}-\xi_{1}^{\prime}\right|,\left|\xi_{2}-\xi_{2}^{\prime}\right|\right\}}{1-|m|^{2}}
$$

The rest of the proof of this case is similar to the last part of the proof of Case 2.1. However, since the phases of $I_{\gamma}(\lambda)$ are non-stationary, the situation is more simple. We can now apply integration by parts directly to $I_{\gamma}(\lambda)$. Applying the arguments of the previous case directly to $I_{\gamma}(\lambda)$ instead of $B_{\gamma}(\lambda)$ (see, in particular (5.17), (5.18), (5.20) and (5.21)), we can derive the bound

$$
\left|I_{\gamma}(\lambda)\right| \leq C \lambda^{-j} \min _{\gamma \in\left\{\gamma_{1}, \gamma_{2}, \gamma_{1}^{\prime}, \gamma_{2}^{\prime}\right\}}\left\{e^{j d(0, \gamma 0)}\right\} e^{-\frac{1}{2}\left(d\left(0, \gamma_{1} 0\right)+d\left(0, \gamma_{1}^{\prime} 0\right)+d\left(0, \gamma_{2} 0\right)+d\left(0, \gamma_{2}^{\prime} 0\right)\right)} .
$$

The rest the proof consists essentially in the argument used to sum the integrals $I_{\gamma, \gamma^{\prime}}(\lambda)$ at the end of the proof of Theorem 5.1. (Compare (5.26) with (5.5).) This gives the bound

$$
\sum I_{\gamma}(\lambda)=\mathcal{O}\left(\lambda^{4 \delta_{\Gamma}-2}\right)
$$


as $\lambda \rightarrow \infty$, where the summation is over all quadruplets $\left(\gamma_{1}, \gamma_{1}^{\prime}, \gamma_{2}, \gamma_{2}^{\prime}\right)$ of distinct elements for which $\psi_{\gamma}$ has no stationary points.

\subsubsection{Case 3: Either $\gamma_{1}^{\prime}=\gamma_{2}^{\prime}$ and $\gamma_{1} \neq \gamma_{2}$ or $\gamma_{1}^{\prime} \neq \gamma_{2}^{\prime}$ and $\gamma_{1}=\gamma_{2}$.}

By multiplying $\nabla_{m} \psi_{\gamma}(m)$ by -1 if necessary, we can suppose without loss of generality that $\xi_{1}^{\prime}=\xi_{2}^{\prime}$ and $\xi_{1} \neq \xi_{2}$. Applying this assumption to (5.11) and (5.12), we get readily that

$$
\nabla_{m} \psi_{\gamma}(m)=\frac{-2\left(\left(\bar{m}-\overline{\xi_{2}}\right)\left(\overline{\xi_{1}}-\overline{\xi_{1}^{\prime}}\right)+\left(\bar{m}-\overline{\xi_{1}}\right)\left(\overline{\xi_{2}}-\overline{\xi_{1}^{\prime}}\right)\right)}{\left(\bar{m}-\overline{\xi_{1}}\right)\left(\bar{m}-\overline{\xi_{2}}\right)\left(\bar{m}-\overline{\xi_{1}^{\prime}}\right)} .
$$

The denominator being bounded from above and from below when $m \in \operatorname{supp} a$, we can restrict our analysis to the numerator. If we multiply the numerator by the complex number $\left(\xi_{1}^{\prime}\right)^{2}$ (a rotation), it becomes

$$
-2\left(\left(\bar{m} \xi_{1}^{\prime}-\overline{\xi_{2}} \xi_{1}^{\prime}\right)\left(\overline{\xi_{1}} \xi_{1}^{\prime}-1\right)+\left(\bar{m} \xi_{1}^{\prime}-\overline{\xi_{1}} \xi_{1}^{\prime}\right)\left(\overline{\xi_{2}} \xi_{1}^{\prime}-1\right)\right) .
$$

Since we are only interested in the complex modulus of the gradient (which is not affected by a rotation), we will suppose without loss of generality that $\xi_{1}^{\prime}=1$ in order to simplify the calculations. This corresponds to rotating the points $\xi_{1}, \xi_{2}$ and $\xi_{1}^{\prime}$ so that $\xi_{1}^{\prime}=1$. The general expression will then be obtained by rotating back once the calculations are completed. With $\xi_{1}^{\prime}=1$, the numerator (5.28) becomes (up to a numerical constant)

$$
\begin{aligned}
\bar{m}\left(\overline{\xi_{1}}+\overline{\xi_{2}}-2\right)+\left(\overline{\xi_{1}}+\overline{\xi_{2}}-2 \overline{\xi_{1} \xi_{2}}\right) & =\bar{m} \overline{\xi_{1} \xi_{2}}\left(\xi_{1}+\xi_{2}-2 \xi_{1} \xi_{2}\right)+\left(\overline{\xi_{1}}+\overline{\xi_{2}}-2 \overline{\xi_{1} \xi_{2}}\right) \\
& =\left(\xi_{1}+\xi_{2}-2 \xi_{1} \xi_{2}\right)\left(\bar{m} \overline{\xi_{1} \xi_{2}}+\frac{\left(\overline{\xi_{1}}+\overline{\xi_{2}}-2 \overline{\xi_{1} \xi_{2}}\right)}{\left(\xi_{1}+\xi_{2}-2 \xi_{1} \xi_{2}\right)}\right) .
\end{aligned}
$$

The value of $\left|\bar{m} \overline{\xi_{1} \xi_{2}}\right|$ being strictly less than 1 and bounded away from 1 uniformly for $\xi_{1}, \xi_{2} \in S^{1}$, when $m$ varies over a compact subset of $\mathbb{D}$, the second term in the product on the right-hand side of (5.29) is larger than some positive constant. Therefore, we have

$$
\left|\nabla_{m} \psi_{\gamma}(m)\right| \geq C\left|\xi_{1}+\xi_{2}-2 \xi_{1} \xi_{2}\right|
$$

for $m \in \operatorname{supp} a$. 
Let us now refine this lower bound by carrying out some elementary calculations:

$$
\begin{aligned}
& \left|\xi_{1}+\xi_{2}-2 \xi_{1} \xi_{2}\right|^{2}=\left|\xi_{1}-1\right|^{2}+\left|\xi_{2}-1\right|^{2}+\xi_{1} \overline{\xi_{2}}+\overline{\xi_{1}} \xi_{2}-\xi_{1}-\overline{\xi_{1}}-\xi_{2}-\overline{\xi_{2}}+2 . \\
& =2\left(\xi_{1}-1\right)\left(\overline{\xi_{1}}-1\right)+2\left(\xi_{2}-1\right)\left(\overline{\xi_{2}}-1\right)-\left(\xi_{1} \overline{\xi_{2}}-1\right)\left(\xi_{2} \overline{\xi_{1}}-1\right) \\
& =2\left|\xi_{1}-1\right|^{2}+2\left|\xi_{2}-1\right|^{2}-\left|\xi_{1}-\xi_{2}\right|^{2} .
\end{aligned}
$$

Since the points $\xi_{1}, \xi_{2}$ and 1 form a triangle, elementary trigonometry can be applied as follows to the right-hand side of the last equation:

$$
\left|\xi_{1}+\xi_{2}-2 \xi_{1} \xi_{2}\right|^{2}=\left|\xi_{1}-1\right|^{2}+\left|\xi_{2}-1\right|^{2}+2\left|\xi_{1}-1\right|\left|\xi_{2}-1\right| \cos \alpha
$$

where $\alpha$ is the angle between the two sides given by $\xi_{1}-1$ and $\xi_{2}-1$ respectively. We decompose $\alpha=\alpha_{1}+\alpha_{2}$, where $\alpha_{i}$ is the angle between $\xi_{i}-1$ and the real axis (the smallest $\alpha_{i}$ is taken to be negative if the two $\xi_{i}$ 's are both on the same side of the real axis). By the trigonometric identity $\cos \left(\alpha_{1}+\alpha_{2}\right)=\cos \left(\alpha_{1}\right) \cos \left(\alpha_{2}\right)-\sin \left(\alpha_{1}\right) \sin \left(\alpha_{2}\right)$ we obtain

$$
\begin{aligned}
\left|\xi_{1}+\xi_{2}-2 \xi_{1} \xi_{2}\right|^{2} & =\left|\xi_{1}-1\right|^{2}-\sin \alpha_{1} \sin \alpha_{2}\left|\xi_{1}-1\right|\left|\xi_{2}-1\right|+\left|\xi_{2}-1\right|^{2} \\
& +2\left(\left|\xi_{1}-1\right| \cos \alpha_{1}\right)\left(\left|\xi_{2}-1\right| \cos \alpha_{2}\right) .
\end{aligned}
$$

Since $\left|\xi_{i}-1\right| \cos \alpha_{i}=1-\operatorname{Re}\left(\xi_{i}\right)$, it is a straightforward elementary calculation to rewrite the last term in the previous equation as $\left|\xi_{1}-1\right|^{2}\left|\xi_{2}-1\right|^{2} / 2$. Therefore,

$$
\left|\xi_{1}+\xi_{2}-2 \xi_{1} \xi_{2}\right|^{2} \geq\left(\left|\xi_{1}-1\right|-\left|\xi_{2}-1\right|\right)^{2}+\frac{\left|\xi_{1}-1\right|^{2}\left|\xi_{2}-1\right|^{2}}{2} \geq \frac{\left|\xi_{1}-1\right|^{2}\left|\xi_{2}-1\right|^{2}}{2}
$$

Hence, for a general $\xi_{1}^{\prime}$ (i.e., after rotating back), we get

$$
\left|\nabla_{m} \psi_{\gamma}(m)\right| \geq C\left|\xi_{1}-\xi_{1}^{\prime}\right|\left|\xi_{2}-\xi_{1}^{\prime}\right|
$$

for all $m \in \operatorname{supp} a$.

On the other hand, differentiating (5.27) (see also (5.29)), we deduce that

$$
\left|\partial_{m}^{\alpha} \nabla_{m} \psi_{\gamma}(m)\right| \leq C_{\alpha} \max \left\{\left|\xi_{1}-\xi_{1}^{\prime}\right|,\left|\xi_{2}-\xi_{1}^{\prime}\right|\right\}
$$


for all $m \in \operatorname{supp} a$. Therefore, a slight modification of the argument leading to (4.13) gives us

$$
\left|\partial_{i} \frac{\partial_{j} \psi_{\gamma}(m)}{\left|\nabla_{m} \psi_{\gamma}(m)\right|^{2}}\right| \leq C_{i} \frac{\max \left\{\left|\xi_{1}-\xi_{1}^{\prime}\right|,\left|\xi_{2}-\xi_{1}^{\prime}\right|\right\}}{\left|\xi_{1}-\xi_{1}^{\prime}\right|^{2}\left|\xi_{2}-\xi_{1}^{\prime}\right|^{2}}=C_{i} \frac{\max \left\{\left|\xi_{1}-\xi_{1}^{\prime}\right|^{-1},\left|\xi_{2}-\xi_{1}^{\prime}\right|^{-1}\right\}}{\left|\xi_{1}-\xi_{1}^{\prime}\right|\left|\xi_{2}-\xi_{1}^{\prime}\right|},
$$

where $i, j=1,2$ and $m \in \operatorname{supp} a$. We can then deduce from Lemma 5.1 , that

$$
\left|\partial_{j} \frac{\partial_{j} \psi_{\gamma}(m)}{\left|\nabla_{m} \psi_{\gamma}(m)\right|^{2}}\right| \leq C_{i} v_{\gamma}
$$

where

$$
v_{\gamma}:=\min \left\{\begin{array}{l}
e^{3 d\left(0, \gamma_{1}^{\prime} 0\right)}, e^{d\left(0, \gamma_{1} 0\right)} e^{d\left(0, \gamma_{2} 0\right)} e^{d\left(0, \gamma_{1}^{\prime} 0\right)}, e^{d\left(0, \gamma_{1} 0\right)} e^{2 d\left(0, \gamma_{1}^{\prime} 0\right)}, e^{d\left(0, \gamma_{2} 0\right)} e^{2 d\left(0, \gamma_{1}^{\prime} 0\right)}, \\
e^{d\left(0, \gamma_{1} 0\right)} e^{d\left(0, \gamma_{2} 0\right)} \max \left\{e^{d\left(0, \gamma_{1} 0\right)}, e^{d\left(0, \gamma_{2} 0\right)}\right\}
\end{array}\right\} .
$$

The phases being non-stationary, we can apply integration by parts directly to the integral $I_{\gamma}(\lambda)$, in the same manner as in $§ 5.2 .2$. By integrating by parts only once, we get the bound

$$
\left|I_{\gamma}(\lambda)\right| \leq C e^{-\frac{1}{2}\left(d\left(0, \gamma_{1} 0\right)+d\left(0, \gamma_{2} 0\right)+2 d\left(0, \gamma_{1}^{\prime} 0\right)\right)} v_{\gamma} \lambda^{-1}
$$

We can now use the decomposition introduced at the end of $§ 5.2 .2$ to sum up the elements. Let $\Gamma^{\prime} \subset \Gamma \times \Gamma \times \Gamma \times \Gamma$ be the set of quadruplets of the form $\left(\gamma_{1}, \gamma_{2}, \gamma_{1}^{\prime}, \gamma_{1}^{\prime}\right)$, where $\gamma_{1}, \gamma_{2}$ and $\gamma_{1}^{\prime}$ are all distinct. Applying Theorem 2.16 as above, we can derive the following analogues of (5.24) and (5.25) respectively:

$$
\sum_{d(0, \gamma 0)>\alpha \log \lambda} e^{-s d(0, \gamma 0)} \leq \int_{\alpha \log \lambda}^{\infty} e^{-s u} d N(u)=\mathcal{O}\left(\lambda^{\alpha\left(\delta_{\Gamma}-s\right)}\right)
$$

and

$$
\sum_{d(0, \gamma 0) \leq \alpha \log \lambda} e^{(t-s) d(0, \gamma 0)}=\mathcal{O}\left(\lambda^{\alpha\left(t+\delta_{\Gamma}-s\right)}\right),
$$

where $s \in\{1 / 2,1\}, t \in\{1,2,3\}$ and $\delta_{\Gamma}<\frac{1}{2}$. Depending on the decomposition $\Gamma_{\alpha_{1}} \times \Gamma_{\alpha_{2}} \times \Gamma_{\alpha_{3}}$, $\alpha_{i} \in\{\leq,>\}$, there are eight different cases to treat. For five of them, inequality (5.30) can be used to obtain the following bounds on $I_{\gamma}(\lambda)$ : 
- If $\left(\alpha_{1}, \alpha_{2}, \alpha_{3}\right)=(\leq, \leq, \leq)$, then

$$
\left|I_{\gamma}(\lambda)\right| \leq C \lambda^{-1} e^{\frac{1}{2} d\left(0, \gamma_{1} 0\right)} e^{\frac{1}{2} d\left(0, \gamma_{2} 0\right)} ;
$$

- If $\left(\alpha_{1}, \alpha_{2}, \alpha_{3}\right)=(>, \leq, \leq)$, then

$$
\left(\left|I_{\gamma}(\lambda)\right| \leq C \lambda^{-1} e^{-\frac{1}{2} d\left(0, \gamma_{1} 0\right)} e^{\frac{1}{2} d\left(0, \gamma_{2} 0\right)} e^{d\left(0, \gamma_{1}^{\prime} 0\right)} ;\right.
$$

- If $\left(\alpha_{1}, \alpha_{2}, \alpha_{3}\right)=(\leq,>, \leq)$, then

$$
\left(\left|I_{\gamma}(\lambda)\right| \leq C \lambda^{-1} e^{\frac{1}{2} d\left(0, \gamma_{1} 0\right)} e^{-\frac{1}{2} d\left(0, \gamma_{2} 0\right)} e^{d\left(0, \gamma_{1}^{\prime} 0\right)} ;\right.
$$

- If $\left(\alpha_{1}, \alpha_{2}, \alpha_{3}\right)=(\leq, \leq,>)$ and

- if $e^{d\left(0, \gamma_{1} 0\right)} \geq e^{d\left(0, \gamma_{2} 0\right)}$, then

$$
\left|I_{\gamma}(\lambda)\right| \leq C \lambda^{-1} e^{\frac{3}{2} d\left(0, \gamma_{1} 0\right)} e^{\frac{1}{2} d\left(0, \gamma_{2} 0\right)} e^{-d\left(0, \gamma_{1}^{\prime} 0\right)} ;
$$

- if $e^{d\left(0, \gamma_{1} 0\right)}<e^{d\left(0, \gamma_{2} 0\right)}$, then

$$
\left|I_{\gamma}(\lambda)\right| \leq C \lambda^{-1} e^{\frac{1}{2} d\left(0, \gamma_{1} 0\right)} e^{\frac{3}{2} d\left(0, \gamma_{2} 0\right)} e^{-d\left(0, \gamma_{1}^{\prime} 0\right)} ;
$$

- If $\left(\alpha_{1}, \alpha_{2}, \alpha_{3}\right)=(>,>, \leq)$, then

$$
\left|I_{\gamma}(\lambda)\right| \leq C \lambda^{-1} e^{-\frac{1}{2} d\left(0, \gamma_{1} 0\right)} e^{-\frac{1}{2} d\left(0, \gamma_{2} 0\right)} e^{2 d\left(0, \gamma_{1}^{\prime} 0\right)} .
$$

Using these bounds in combination with (5.31) and (5.32), we can sum up the elements for the cases above. For each of them we have $\sum\left|I_{\gamma}(\lambda)\right|=\mathcal{O}\left(\lambda^{\alpha\left(3 \delta_{\Gamma}+1\right)-1}\right)$.

For the last three cases of $\left(\alpha_{1}, \alpha_{2}, \alpha_{3}\right)$, we do not integrate by parts. We use instead the estimate

$$
\left|I_{\gamma}(\lambda)\right| \leq C e^{-\frac{1}{2} d\left(0, \gamma_{1} 0\right)} e^{-\frac{1}{2} d\left(0, \gamma_{2} 0\right)} e^{-d\left(0, \gamma_{1}^{\prime} 0\right)},
$$


which is obtained trivially by bounding the integrand of $I_{\gamma}(\lambda)$ with the use of Lemma 2.19. For $(\leq,>,>)$ and $(>, \leq,>)$, the bound (5.31) in combination with

$$
\sum_{d(0, \gamma 0) \leq \alpha \log \lambda} e^{-\frac{1}{2} d(0, \gamma 0)}=\mathcal{O}\left(\lambda^{\alpha \delta_{\Gamma}}\right)
$$

provides us with

$$
\sum\left|I_{\gamma}(\lambda)\right|=\mathcal{O}\left(\lambda^{\alpha\left(3 \delta_{\Gamma}-\frac{3}{2}\right)}\right)
$$

Notice that (5.33) was obtained by applying Theorem 2.16 and $e^{-\frac{1}{2} d(0, \gamma 0)} \leq 1$. For $(>,>,>)$, we apply (5.31) to each of $\gamma_{1}, \gamma_{2}$ and $\gamma_{1}^{\prime}$, which gives

$$
\left|I_{\gamma}(\lambda)\right| \leq C e^{-\frac{1}{2} d\left(0, \gamma_{1} 0\right)} e^{-\frac{1}{2} d\left(0, \gamma_{2} 0\right)} e^{-d\left(0, \gamma_{1}^{\prime} 0\right)}
$$

Hence $\sum\left|I_{\gamma}(\lambda)\right|=\mathcal{O}\left(\lambda^{\alpha\left(3 \delta_{\Gamma}-2\right)}\right)$. If we set $\alpha=\frac{1}{3}$ in any of the previous bounds, we obtain

$$
\sum_{\gamma \in \Gamma^{\prime}}\left|I_{\gamma}(\lambda)\right|=\mathcal{O}\left(\lambda^{\delta_{\Gamma}-\frac{1}{2}}\right)
$$

as $\lambda \rightarrow \infty$.

Since all the cases have been treated and the bound $\mathcal{O}\left(\lambda^{\delta_{\Gamma}-\frac{1}{2}}\right)$ is the largest one, we have

$$
I_{2}^{4}(\lambda, \xi)=\int_{X} a(m) f_{4}(m, \xi) d v_{X}(m)+\mathcal{O}\left(\lambda^{\delta_{\Gamma}-\frac{1}{2}}\right)
$$

The independence of the implied constant on $\xi \in \partial \bar{X}$ follows from the explanation at the end of $§ 4.1 .3$ and from the fact that the argument leading to Theorem 5.2 is also independent of $\xi$. The proof of the theorem is then completed.

\subsection{Proof of Corollary 1.5}

We use a slight modification of the argument appearing in the proof of Theorem 1.2 to treat the remaining terms in the expansion of $\left(E\left(s_{\lambda} ; m, \xi\right) \pm \overline{E\left(s_{\lambda} ; m, \xi\right)}\right)^{4}$.

Proposition 5.3. Let $X=\Gamma \backslash \mathbb{H}^{2}$ be a convex co-compact hyperbolic surface with $\delta_{\Gamma}<1 / 2$. Let $a \in C_{0}^{\infty}(X)$ and let $E(s ; \cdot, \xi)$ be an Eisenstein series with $\xi \in \partial \bar{X}$. Then for $k=0,1,3$ 
or 4 ,

$$
\int_{X} a(m)\left(E\left(s_{\lambda} ; m, \xi\right)\right)^{k}\left(\overline{E\left(s_{\lambda} ; m, \xi\right)}\right)^{4-k} d v_{X}(m)=\mathcal{O}\left(\lambda^{-\frac{1}{d}}\right)
$$

as $\lambda \rightarrow \infty$, where $d=16$ if $k=0$ or 4 and $d=18$ if $k=1$ or 3 .

Sketch of the proof. The proof of Theorem 1.2 does not apply directly when $p=4$. A specific choice of $\gamma_{1}, \gamma_{2}, \gamma_{3}, \gamma_{4} \in \Gamma$ can make the gradient of $\psi_{\gamma}$ vanish everywhere on a parametrized line $x \mapsto(x, y) \in \mathbb{D}$, for some $y \in(-1,1)$, thus preventing the constant $C_{m}$ introduced in the proof of this theorem to be positive.

If $k=0$ or 4 , the geometrical argument used in Theorem 1.3 applies directly to conclude that in order for $\nabla \psi_{\gamma}(m)$ to vanish, $m$ must lie on the intersection of two geodesics joining the two pairs of points $\left(\gamma_{i_{1}}^{-1} \xi, \gamma_{i_{2}}^{-1} \xi\right)$ and $\left(\gamma_{i_{3}}^{-1} \xi, \gamma_{i_{4}}^{-1} \xi\right)$ respectively, for some choice of $i_{1}, i_{2}, i_{3}, i_{4} \in$ $\{1,2,3,4\}$. It follows that for $\nabla \psi_{\gamma}(m)$ to vanish on an Euclidean line, we must have $i_{1}=i_{3}$ and $i_{2}=i_{4}$, or $i_{1}=i_{4}$ and $i_{2}=i_{3}$. Moreover, this line is horizontal if and only if $i_{1}=i_{3}= \pm 1$ and $i_{2}=i_{4}=\mp 1$ ( or $i_{1}=i_{4}= \pm 1$ and $i_{2}=i_{3}=\mp 1$, respectively).

Notice that this issue would not arise if instead of considering the whole set $\mathbb{S}^{4}$, we apply the argument to a closed subset $S_{1} \subset \mathbb{S}^{4}$, for which it always happens that one of the $\gamma_{j}^{-1} \xi^{\prime}$ s is bounded away from the points \pm 1 . Interchanging $x$ and $y$ in the proof, the argument can be applied again to the closure (in $\mathbb{R}^{2}$ ) of the complement of $S_{1}$ (in $\mathbb{S}^{4}$ ). We then get two different constants $C$ in (4.17). The statement follows by taking the largest one and by applying the rest of the proof of Theorem 1.2 .

The cases where $k=1$ or 3 are treated similarly, noticing that in order for $\nabla \psi_{\gamma_{1, \pm}, \gamma_{2, \pm}, \gamma_{3, \pm}, \gamma_{\mp}}$ to vanish on a horizontal line, we must have $\gamma_{\mp}^{-1} \xi=\gamma_{i_{1}, \pm}^{-1} \xi, \gamma_{i_{2}, \pm}^{-1} \xi=1$ and $\gamma_{i_{3}, \pm}^{-1} \xi=-1$ for some choice of $i_{1}, i_{2}, i_{3} \in\{1,2,3\}$. We can then take $S_{1} \subset \mathbb{S}^{4}$ to be a closed subset for which it always happens that two of the $\gamma_{j, \pm}^{-1} \xi$ 's are bounded away from \pm 1 . The statement follows as described above by applying the argument a second time to the closure of the complement of this set, with $x$ replaced by $y$. 
Corollary 1.5 is then a straightforward application of Theorem 1.3 and Proposition 5.3 to the expansion of $\left(E\left(s_{\lambda} ; m, \xi\right) \pm \overline{E\left(s_{\lambda} ; m, \xi\right)}\right)^{4}$.

We conclude this subsection with a remark about the second moment of the real and imaginary parts of Eisenstein series. The high-energy limit of the restricted $L^{2}$-norm of the Eisenstein series is the content of Theorem 5.2. In order to compute the second moment of the real and imaginary parts, one must also consider the limit as $\lambda \rightarrow \infty$ of the integrals $I_{0}^{2}(\lambda)$ and $I_{2}^{2}(\lambda)$. By Theorem 1.1, we know that these integrals vanish at high-energy. The rate of convergence can be estimated by adapting the proof of Theorem 1.2. More precisely, one can use a geometrical argument, similar to the one invoked in the proof of Theorem 1.3, to conclude that, in this case, the zero set of $\nabla \psi_{\gamma, \gamma^{\prime}}(m)$ corresponds to the geodesic lying between the two points $\gamma^{-1} \xi$ and $\gamma^{\prime-1} \xi$ on the unit sphere. An argument along the lines of the proof of Proposition 5.3 (for the cases $k=0$ and $k=4$ ) would then apply. This gives us the error term $\mathcal{O}\left(\lambda^{-\frac{1}{8}}\right)$ for both $I_{0}^{2}(\lambda)$ and $I_{2}^{2}(\lambda)$. Equation (1.8) follows directly. 


\section{CHAPTER 6 \\ Conclusion}

The problem of determining the high-energy limit of the moments of the Eisenstein series on convex co-compact hyperbolic manifolds has been solved completely in the case where the dimension of the limit set is less than $\frac{n}{2}$. However, the question about the rate of convergence is yet far from being completely solved, not to mention finding precise asymptotics for any of the moments.

The polynomial error terms obtained for the odd-order moments are not sharp in general. This is essentially due to the generality of our approach, which does not take into account the properties of the stationary points involved with enough specificity. On the other hand, the direct approach followed in $\S 4.2$ and in Chapter 5 to treat the third and the fourth moments of the Eisenstein series on surfaces does not seem to generalize easily to higher moments. The larger the power of the Eisenstein series, the more complicated the expression of the gradient of the phase function. Consequently, the calculations involved in the determination of its zero set become quickly very tedious. Moreover, even a precise knowledge of the stationary points is not sufficient to guarantee the success of this approach. The method developed here depends strongly on the geometry of the problem and on the simplicity of the stationary points involved. For example, a straightforward generalization could not be expected when the phase is stationary at more than one point.

For much the same reasons, it does not seem practicable to adapt the proofs of these two special cases to manifolds of higher dimensions. For example, when $n=2$, the gradient of the phase functions appearing in the calculations of the restricted $L^{4}$-norm would generally not vanish at one point, but on a (1-dimensional) curve. New ideas would then be required to resolve this issue which does not arise on surfaces (i.e., when $n=1$ ). 
On the other hand, it does not seem that a general argument of the same nature as the one leading to Theorem 1.2 would be suitable for the even-order moments. Indeed, the "middle-term", i.e., $I_{p / 2}^{p}(\lambda)$, gives rise to oscillatory integrals that have phase functions for which the gradient cannot be bounded from below uniformly in $\gamma$, contrary to the odd case. A more precise knowledge of the gradient of the phase functions appears then to be necessary if one wants to follow this approach. However, as discussed above, due to the complexity of the calculations involved, such information does not seem to be easily attainable when $p$ is large. For all of these reasons, we think that the methods developed here have reached their limit as far as the rate of convergence is concerned.

A natural generalization of the problem addressed in this work would be to consider the case where the dimension of the limit set is larger than $n / 2$. However, under these circumstances the Eisenstein series do not lift to the absolutely convergent series provided by Lemma 2.20 . We then expect that an approach of considerably different nature would be necessary to tackle this problem.

In view of Theorem 1.1 and Corollary 3.3, a natural direction for further research is the question mentioned in $\S 1.1$ about the possible existence of a random variable $F$ to which the family of random variables $F_{\lambda}$ would converge in distribution. Starting from the knowledge of $\lim _{\lambda \rightarrow \infty} \mathbb{E}\left(F_{\lambda}^{p}\right)$ for all $p \geq 1$, one could now attempt to identify a probability law whose moments and the ones of the Eisenstein series at high-energy coincide. However, since the limits $\lim _{\lambda \rightarrow \infty} \mathbb{E}\left(F_{\lambda}^{p}\right)$ depend (at least a priori) on the choice of the manifold $X$, finding a general expression for such a probability distribution is not expected to be straightforward. 


\section{REFERENCES}

[1] Andrews G. E. The Theory of Partitions. Encyclopedia of Mathematics and its Applications 2. Cambridge University Press, 1976.

[2] Beardon, A. F. "The exponent of convergence of Poincaré series." Proc. London Math. Soc. 18 (1968): 461-483.

[3] Beardon, A. F. "Inequalities for certain Fuchsian groups." Acta Math. 127 (1971): 221258.

[4] Beardon, A. F. The Geometry of Discrete Groups. Graduate Text in Mathematics 91. New York: Springer-Verlag, 1983.

[5] Bérard, P. H. Spectral Geometry: Direct and Inverse Problems. Lecture Notes in Mathematics 1207. Berlin and New York: Springer-Verlag, 1986.

[6] Berry, M. "Regular and irregular semiclassical wavefunctions." Journal of Physics A: Mathematical and General 10 (1977): 2083-2091.

[7] Borthwick, D. Spectral Theory of Infinite-Area Hyperbolic Surfaces. Progress in Mathematics 256. Boston: Birkhäuser, 2007.

[8] Buser, P. Geometry and Spectra of Compact Riemann Surfaces. Modern Birkhäuser Classics. Boston: Birkhäuser, 1992.

[9] Button, J. "All Fuchsian Schottky groups are classical Schottky groups." Geometry 8 Topology Monographs 1 (1998): 117-125.

[10] Canzani, Y., D. Jakobson, and J. Toth. "On the distribution of propagated Schrödinger eigenfunctions." Jour. of Spectral Theory 4, no. 2 (2014): 283-307.

[11] Chavel, I. Eigenvalues in Riemannian Geometry. Pure and Applied Mathematics 115. Orlando: Academic Press, 1984.

[12] Colin de Verdière, Y. "Ergodicité et fonctions propres du Laplacien", Comm. Math. Phys. 102 (1985), 497-502.

[13] Deshouillers, J.M., Iwaniec, H., Phillips, R.S., and P. Sarnak. "Maass cusp forms." Proc. Nat. Acad. Sci. U.S.A. 82 (1985): 3533-3534.

[14] Guillarmou, C. "Meromorphic properties of the resolvent for asymptotically hyperbolic manifolds." Duke Math. J. 129, no. 1 (2005): 1-37. 
[15] Guillarmou, C. and R. Mazzeo. "Resolvent of the Laplacian on geometrically finite hyperbolic manifolds." Inventiones Math. 187, no. 1 (2012): 99-144.

[16] Guillarmou, C. and F. Naud. "Equidistribution of Eisenstein series on convex cocompact hyperbolic manifold." American Journal of Mathematics 136, no. 2 (2014): 445-479.

[17] Guillopé, L. and M. Zworski. "Polynomial Bounds on the number of resonances for some complete spaces of constant negative curvature near infinity." Asymp. Anal. 11 (1995): $1-22$.

[18] Hejhal, D. A. and B.N. Rackner. "On the topography of Maass waveforms for PSL(2,Z)." Experiment. Math. 1, no. 4 (1992): 275-305.

[19] Hopf, E. "Statistik der geodätischen Linien in Mannigfaltigkeiten negativer Krümmung." Ber. Verh. Sächs. Akad. Wiss. Leipzig 91 (1939), 261-304.

[20] Iwaniec, H. Spectral methods of automorphic forms. Second edition. Graduate Studies in Mathematics 53. Providence: American Mathematical Society, 2002. 53.

[21] Iwaniec H. and P. Sarnak. " $L^{\infty}$ norms of eigenfunctions of arithmetic surfaces", Annals of Math. (2) 141 (1995): 301-320.

[22] Lax, P. D. and R. S. Phillips. "The asymptotic distribution of lattice points in Euclidean and non-Euclidean spaces." J. Funct. Anal. 46, no. 3 (1982): 280-350.

[23] Lax, P. D. and R. S. Phillips. "Translation representations for automorphic solutions of the wave equation in non-Euclidean spaces. I." Communications on Pure and Applied Mathematics Vol. XXXVII, (1984): 303-328.

[24] Lax, P. D. and R. S. Phillips. "Translation representations for automorphic solutions of the wave equation in non-Euclidean spaces; The case of finite volume." Transactions of the American Mathematical Society 289, no. 2 (1985): 715-735.

[25] Luo, W., and P. Sarnak. "Quantum ergodicity of Eigenfunctions on $\mathrm{PSL}_{2}(\mathbb{Z}) \backslash \mathbb{H}^{2}$." Publ. Math. de l'IHES 81 (1995): 207-237.

[26] Ma, T. W. "Higher Chain Formula proved by Combinatorics." The Electronic Journal of Combinatorics 16, \# N21 (2009): 1-7.

[27] Maskit, B. "A characterization of Schottky groups." J. Analyse Math 19 (1967): $227-230$.

[28] Milićević, D. "Large values of eigenfunctions on arithmetic hyperbolic surfaces." Duke Mathematical Journal 155, no. 2 (2010): 365-401.

[29] Mazzeo, R. R. and R. B. Melrose. "Meromorphic Extension of the Resolvent on Complete Spaces with Asymptotically Constant Negative Curvature." J. Funct. Anal. 75 (1987): 260-310. 
[30] Patterson, S. J. "The limit set of a Fuchsian group." Acta Math. 136, no. 3-4 (1976): 241-273.

[31] Patterson, S. J. "On a lattice-point problem in hyperbolic space and related questions in spectral theory." Arxiv Math. 26 (1988): 167-172.

[32] Phillips, R. S., and P. Sarnak. "The Weyl Theorem and the Deformation of Discrete Groups", Comm. Pure. Appl. Math. 38 (1985): 853-866.

[33] Poincaré, H. "Mémoire sur les fonctions Fuchsiennes." Acts Math. 1 (1882): 193-294.

[34] Ratcliffe, J. G. Foundations of Hyperbolic Manifolds. Second Edition. Graduate Texts in Math. 149. New York: Springer-Verlag, 2006.

[35] Sarnak, P. "Arithmetic quantum chaos." Israel Math. Conf. Proc. 8 Bar-Ilan Univ. Ramat Gan (1995): 183-236.

[36] Sarnak, P. "Spectra of hyperbolic surfaces." Bull. Amer. Math. Soc. (N.S.) 40, no. 4 (2003): 441-478.

[37] Selberg, A. "Harmonic analysis and discontinuous groups in weakly symmetric Riemannian spaces with applications to Dirichlet series." J. Indian Math. Soc. 20 (1956), 47-87.

[38] Schnirelman, A.I. "Ergodic properties of eigenfunctions." Usp. Math. Nauk. 29 (1974), 181-182.

[39] Spinu, F. "The L4 norm of the Eisenstein series." Ph.D. Thesis, Princeton University (2003).

[40] Sullivan, D. "The density at infinity of a discrete group of hyperbolic motions." Publ. Math. de l'IHES. 50 (1979): 171-202.

[41] Venkov, A. Spectral theory of automorphic functions and its applications. Kluwer, 1990.

[42] Watson, T. "Rankin triple products and quantum chaos." Ph.D. Thesis, Princeton University (2002).

[43] Weyl, H. "On the Volume of Tubes." American Journal of Mathematics 61, no. 2 (1939): 461-472.

[44] Zedek, M. "Continuity and Location of Zeros of Linear Combinations of Polynomials." Proc. Amer. Math. Soc. 16 (1965): 78-84.

[45] Zelditch, S. "Uniform distribution of eigenfunctions on compact hyperbolic surfaces" Duke Math. J. 55 (1987): 919-941. 